\title{
Variação temporal da elasticidade no método da fronteira imersa
}

\author{
Rudimar Luiz Nós \\ DISSERTAÇÃO APRESENTADA \\ $\mathrm{AO}$ \\ INSTITUTO DE MATEMÁTICA E ESTATÍSTICA \\ DA \\ UNIVERSIDADE DE SÃO PAULO \\ PARA \\ OBTENÇÃO DO GRAU DE MESTRE \\ EM \\ MATEMÁTICA APLICADA \\ Área de concentração: Matemática Aplicada - Análise Numérica \\ Orientador: Profa. Dra. Joyce da Silva Bevilacqua
}

São Paulo, 3 de janeiro de 2002 


\section{Variação temporal da elasticidade no método da fronteira imersa}

Este exemplar corresponde à redação final da dissertação devidamente corrigida e defendida por Rudimar Luiz Nós e aprovada pela comissão julgadora.

São Paulo, 3 de janeiro de 2002.

Banca examinadora:

- Prof. Dra. Joyce da Silva Bevilacqua (Presidente) - IME/USP

- Prof. Dr. Alexandre Megiorin Roma - IME/USP

- Prof. Dr. Júlio Romano Meneghini - EP/USP 

À vontade, ao esforço e à perseverança, suplantadores de obstáculos e limitações 



\section{Agradecimentos}

À Joyce, por acreditar e confiar

Ao Santos e ao Massaishi, pela paciência e prestatividade

À Olga, pela amizade e companheirismo

À família e aos amigos, pela compreensão e apoio

Ao IME-USP e ao CEFET-PR, pela possibilidade de aprender 



\section{RESUMO}

Inúmeros fenômenos em Biologia e Medicina são modelados por fibras elásticas completamente imersas em um fluido, como por exemplo a locomoção de microorganismos ou o fluxo sangüíneo através de uma válvula cardíaca. Expansões ou contrações da fibra podem ser simuladas pelo método da fronteira imersa desenvolvido por Peskin. A expansão da fibra é controlada pelo valor da constante elástica e pela inserção de fontes e sorvedouros no domínio de estudo da interação fluido-fibra. Neste trabalho emprega-se o método da fronteira imersa para modelar a expansão do alvéolo pulmonar durante a inspiração. A resistência e a complacềncia pulmonar, assim como a ação da substância surfactante, são representadas por uma função que varia temporalmente a elasticidade ao longo de uma porção da fibra e pela distribuição de um número adequado de fontes e sorvedouros no domínio computacional. 


\section{ABSTRACT}

Many phenomena in Biology and Medicine are modeled by elastic fibers immersed in a fluid, as locomotion of microorganisms or blood flow through a cardiac valve. Expansions or contractions of the fiber can be simulated using the immersed boundary method developed by Peskin. The expansion of the fiber is controlled by value of the elastic constant and sources and sinks inserted in the domain of study fluid-fiber interaction. In this work we use the immersed boundary method to model the expansion of pulmonary alveoli during inspiratory time. Resistance, compliance and surfactant substance are represented by an elastic function acting on the boundary and an adequate number of sources and sinks distributed in the computational domain. 


\section{Índice}

Lista de Figuras $\quad$. XV

Lista de Tabelas $\quad$ xix

Introdução

1 Aspectos mecânicos e fisiológicos da respiração $\quad 5$

1.1 Pressões . . . . . . . . . . . . . . . . . . . . . 9

1.2 Resistência das vias aéreas . . . . . . . . . . . . . . . . . 11

1.3 Complacência . . . . . . . . . . . . . . . . . . . 12

1.4 Surfactante - Tensão superficial . . . . . . . . . . . . . . . . . 14

2 Modelo matemático $\quad 17$

2.1 Variação volumétrica do alvéolo na inspiração . . . . . . . . . . . . 19

$2.2 \mathrm{O}$ método da fronteira imersa . . . . . . . . . . . . . . . . 21

2.2 .1 Equações . . . . . . . . . . . . . . . . . . . . 22

2.2.2 Introdução de fontes e sorvedouros . . . . . . . . . . . . . 28

2.2 .3 Força elástica . . . . . . . . . . . . . . . . . . 31

3 Discretização das equações do modelo $\quad 35$

3.1 Equações do fluido . . . . . . . . . . . . . . . . . . . . . . . 41

3.2 Equações de interação fluido-fibra . . . . . . . . . . . . . . . . . . . . 44 
3.3 Algoritmo . . . . . . . . . . . . . . . . . . . . . . 44

3.4 Introdução de fontes e sorvedouros . . . . . . . . . . . . . 46

3.5 Algoritmo para introdução de fontes e sorvedouros . . . . . . . . . . . . . . 54

3.6 Força exercida pela fronteira . . . . . . . . . . . . . . . . 57

4 Modelagem da fronteira elástica $\quad 61$

4.1 Fontes e sorvedouros . . . . . . . . . . . . . . . . . . . . 62

4.2 Fronteira elástica . . . . . . . . . . . . . . . . . 75

4.2 .1 Ajuste do número $k$ de fontes e sorvedouros . . . . . . . . 78

4.3 Valores fisiológicos para a densidade $\rho$ e a viscosidade $\mu$ do fluido . . . . . 81

$4.4 \mathrm{Um}$ modelo para dois alvéolos . . . . . . . . . . . . . . . . . . 83

$\begin{array}{llr}5 & \text { Simulações } & 87\end{array}$

5.1 Simulação de um alvéolo . . . . . . . . . . . . . . . . . 87

5.1.1 Densidade e viscosidade não fisiológicos . . . . . . . . . . . 87

5.1 .2 Densidade e viscosidade fisiológicos . . . . . . . . . . . . 94

5.2 Simulação de dois alvéolos . . . . . . . . . . . . . . . . 96

5.2.1 Expansões simétricas . . . . . . . . . . . . . . . . . 96

5.2 .2 Expansões assimétricas . . . . . . . . . . . . . . . . 100

Conclusão

A Fluxo laminar e turbulento

B Número de Reynolds

C Algoritmo de Thomas

D Avaliação da pressão $\bar{P}_{m}$, da resistência $R_{m}$ e do suporte $h_{o}$ para $k=5113$ 


\section{Lista de Figuras}

1.1 Alvéolos pulmonares - Hopkins [17], Enciclopédia Encarta [7] . . . . . . . . . . . . . 6

1.2 Alvéolos em corte tridimensional e bidimensional - Hopkins [17] . . . . . . . . . . . . 6

1.3 Complexo capilar que circunda os alvéolos - Corpo Humano [5] . . . . . . . . . . . . 7

1.4 Contribuição do surfactante à estabilidade do alvéolo - Hopkins [17] . . . . . . . . . . 15

2.1 Modelo matemático para o alvéolo pulmonar f . . . . . . . . . . . . . . 17

2.2 Modelo matemático com fontes e sorvedouros . . . . . . . . . . . . . . . 18

2.3 Volumes associados ao modelo . . . . . . . . . . . . . . . . . . . . . . . . 19

2.4 Formulação Euleriana-Lagrangeana do método da fronteira imersa . . . . . . . . . . . 24

2.5 Forças atuantes em um trecho arbitrário da fibra . . . . . . . . . . . . . 25

2.6 Força elástica em uma região arbitrária $\mathrm{R} \ldots \ldots \ldots$. . . . . . . . . . . . . 26

2.7 Ação da m-ésima fonte e do m-ésimo sorvedouro . . . . . . . . . . . . . . . . . 30

3.1 Malha computacional para $\mathrm{n}=5 \mathrm{e} h=0.2 \ldots \ldots \ldots \ldots \ldots$

3.2 Delta de Dirac unidimensional com centro em $x=0.5$ e bidimensional com centro em

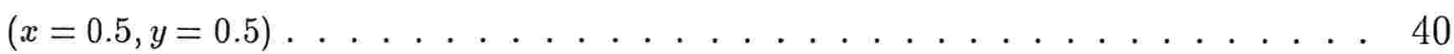

3.3 Interpolações efetuadas com o emprego do delta de Dirac: (A) distribuição da densidade de força elástica $f_{l}$ exercida pelo ponto $X_{l}$ da fibra para os pontos $x_{i, j}$ do fluido pertencentes à região interna ao quadrado de lado $4 h_{o}$ do qual $X_{l}$ é o centro; (B) cálculo da velocidade do ponto $X_{l}$ da fibra a partir da velocidade $u_{i, j}$ dos pontos $x_{i, j}$ do fluido pertencentes à região interna ao quadrado de lado $4 h_{o}$ do qual $X_{l}$ é o centro . . . . . . 41

3.4 Algoritmo estruturado do método da fronteira imersa . . . . . . . . . . . . . . . 45

3.5 Função $\Psi_{m}(x)$ para três fontes e três sorvedouros $\ldots \ldots \ldots$. . . . . . . . . . 49 
3.6 Algoritmo estruturado do método da fronteira imersa com a inclusão de $k$ fontes e $k$ sorvedouros ........................ . . . 55

3.7 Modelo para um alvéolo pulmonar . . . . . . . . . . . . . . . . . . . . . 57

4.1 Comportamento da geometria inicial da fronteira imersa para $k=2,3,5,7$ e 9 após $t \approx 0.17$. . . . . . . . . . . . . . . . . . . . . 66

$4.2 R_{m}=10^{5} \frac{\mathrm{g}}{\mathrm{cm}^{3} \cdot \mathrm{s}}, \bar{P}_{m}=10^{3} \frac{\mathrm{g}}{\mathrm{cm} \cdot \mathrm{s}^{2}}, h_{o}=\frac{1}{32}$ e $\mathrm{k}_{\max }=10^{6} \frac{d y n}{\mathrm{~cm}} . . . . . . . . .68$

$4.3 R_{m}=10^{7} \frac{g}{\mathrm{~cm}^{3} . s}, \bar{P}_{m}=10^{3} \frac{\mathrm{g}}{\mathrm{cm} \cdot \mathrm{s}^{2}}, h_{o}=\frac{1}{32}$ e $\mathrm{k}_{\max }=10^{6} \frac{d y n}{\mathrm{~cm}} \ldots . . . . . . . .70$

$4.4 R_{m}=10^{6} \frac{g}{c m^{3} . s}, \bar{P}_{m}=10^{3} \frac{g}{c m . s^{2}}, h_{o}=\frac{1}{32}$ e $\mathrm{k}_{\max }=10^{6} \frac{d y n}{c m} \ldots . . . . . . .71$

$4.5 \quad R_{m}=10^{4} \frac{g}{\mathrm{~cm}^{3} . \mathrm{s}}, \bar{P}_{m}=10^{2} \frac{\mathrm{g}}{\mathrm{cm} \cdot \mathrm{s}^{2}}, h_{o}=\frac{1}{64} \mathrm{e} \mathrm{k}_{\max }=10^{6} \frac{\mathrm{dyn}}{\mathrm{cm}} \ldots . . . . . . .73$

$4.6 \quad R_{m}=10^{4} \frac{\mathrm{g}}{\mathrm{cm}^{3} \cdot \mathrm{s}}, \bar{P}_{m}=10^{3} \frac{\mathrm{g}}{\mathrm{cm} \cdot \mathrm{s}^{2}}, h_{o}=\frac{1}{32} \mathrm{e} \mathrm{k}_{\max }=10^{6} \frac{\mathrm{dyn}}{\mathrm{cm}} . . . . . . . . .74$

4.7 Modelo para dois alvéolos pulmonares . . . . . . . . . . . . . . . . . . . . . 83

$5.1 k^{*}[i]=\operatorname{sen}\left(e^{k[i] n \Delta t}\right), R_{m}=10^{5} \frac{g}{\mathrm{~cm}^{3} \cdot \mathrm{s}}, \bar{P}_{m}=10^{3} \frac{\mathrm{g}}{\mathrm{cm} \cdot \mathrm{s}^{2}}, h_{o}=\frac{1}{32}, \rho=1 \frac{g}{\mathrm{~cm}^{3}}, \mu=$ $10^{-2} \frac{g}{c m . s}, k=9, N=32$, Variação de área: $125.41 \%$ após $t=1.0 \mathrm{~s}, 156.34 \%$ após $t=1.5 \mathrm{~s}$, Fontes centradas em $(0.2,0.5)$, Sorvedouros centrados em $(0.55,0.1)(0.65,0.15)$ $(0.75,0.2)(0.85,0.3)(0.9,0.5)(0.85,0.7)(0.75,0.8)(0.65,0.85)(0.55,0.9) \quad$. . . . . . .

$5.2 k[i]=1.0, R_{m}=10^{5} \frac{g}{\mathrm{~cm}^{3} \cdot s}, \bar{P}_{m}=10^{3} \frac{\mathrm{g}}{\mathrm{cm} \cdot \mathrm{s}^{2}}, h_{o}=\frac{1}{32}, \rho=1 \frac{\mathrm{g}}{\mathrm{cm}^{3}}, \mu=10^{-2} \frac{\mathrm{g}}{\mathrm{cm} \cdot \mathrm{s}}$, $k=9, N=32$, Variação de área: $125 \%$ após $t=1.0 \mathrm{~s}, 154.98 \%$ após $t=1.5 \mathrm{~s}$, Fontes centradas em $(0.2,0.5)$, Sorvedouros centrados em $(0.55,0.1)(0.65,0.15)(0.75,0.2)$ $(0.85,0.3)(0.9,0.5)(0.85,0.7)(0.75,0.8)(0.65,0.85)(0.55,0.9)$. . . . . . . . . . 90

$5.3 k[i]=0.1, R_{m}=10^{5} \frac{\mathrm{g}}{\mathrm{cm}^{3} . \mathrm{s}}, \bar{P}_{m}=10^{3} \frac{\mathrm{g}}{\mathrm{cm} \cdot \mathrm{s}^{2}}, h_{o}=\frac{1}{32}, \rho=1 \frac{\mathrm{g}}{\mathrm{cm}^{3}}, \mu=10^{-2} \frac{\mathrm{g}}{\mathrm{cm} . \mathrm{s}}$, $k=9, N=32$, Variação de área: $148.86 \%$ após $t=1.0 \mathrm{~s}, 197.32 \%$ após $t=1.5 \mathrm{~s}$, Fontes centradas em $(0.2,0.5)$, Sorvedouros centrados em $(0.55,0.1)(0.65,0.15)(0.75,0.2)$ $(0.85,0.3)(0.9,0.5)(0.85,0.7)(0.75,0.8)(0.65,0.85)(0.55,0.9)$. . . . . . . . . .

$5.4 k^{*}[i]=e^{(3.6) k[i] n \Delta t}, R_{m}=10^{5} \frac{\mathrm{g}}{\mathrm{cm}^{3} \cdot \mathrm{s}}, \bar{P}_{m}=10^{3} \frac{\mathrm{g}}{\mathrm{cm} \cdot \mathrm{s}^{2}}, h_{o}=\frac{1}{32}, \rho=1 \frac{\mathrm{g}}{\mathrm{cm}^{3}}, \mu=$ $10^{-2} \frac{g}{\mathrm{~cm} . s}, k=9, N=32$, Variação de área: $84.36 \%$ após $t=1.0 \mathrm{~s}, 60.18 \%$ após $t=1.5 \mathrm{~s}$, Fontes centradas em $(0.2,0.5)$, Sorvedouros centrados em $(0.55,0.1)(0.65,0.15)$ $(0.75,0.2)(0.85,0.3)(0.9,0.5)(0.85,0.7)(0.75,0.8)(0.65,0.85)(0.55,0.9) \quad$. . . . . .

$5.5(k[i] n \Delta t-5)^{2}$ para $0<n \Delta t \leq 1.0,(k[i] n \Delta t-10)^{2}$ para $1.0<n \Delta t \leq 1.5, R_{m}=$ $10^{5} \frac{\mathrm{g}}{\mathrm{cm}^{3} \cdot \mathrm{s}}, \bar{P}_{m}=10^{3} \frac{\mathrm{g}}{\mathrm{cm} \cdot \mathrm{s}^{2}}, h_{o}=\frac{1}{64}, \rho=1.21 \times 10^{-3} \frac{\mathrm{g}}{\mathrm{cm}^{3}}, \mu=1.9 \times 10^{-4} \frac{\mathrm{g}}{\mathrm{cm} \cdot \mathrm{s}}, k=9, N=$ 128, Variação de área: $128.76 \%$ após $t=1.0 \mathrm{~s}, 157.28 \%$ após $t=1.5 \mathrm{~s}$, Fontes centradas em $(0.2,0.5)$, Sorvedouros centrados em $(0.55,0.1)(0.65,0.15)(0.75,0.2)(0.85,0.3)(0.9,0.5)$

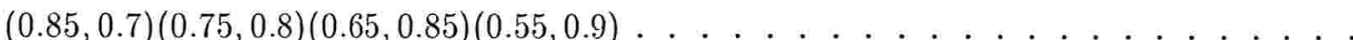


$5.6 k^{*}[i]=\operatorname{sen}\left(e^{k[i] n \Delta t}\right), R_{m}=10^{5} \frac{g}{\mathrm{~cm}^{3} . s}, \bar{P}_{m}=10^{3} \frac{g}{\mathrm{~cm} \cdot \mathrm{s}^{2}}, h_{o}=\frac{1}{32}, \rho=1 \frac{\mathrm{g}}{\mathrm{cm}}, \mu=$ $10^{-2} \frac{\mathrm{g}}{\mathrm{cm} . \mathrm{s}}, k=9, N=32$, Variação de área: $60.57 \%$ após $t=1.0 \mathrm{~s}, 64.99 \%$ após $t=1.5 s$, Fontes centradas em $(0.2,0.5)$, Sorvedouros centrados em $(0.55,0.1)(0.65,0.15)$ $(0.75,0.2)(0.85,0.3)(0.9,0.5)(0.85,0.7)(0.75,0.8)(0.65,0.85)(0.55,0.9) \quad \ldots \ldots . .97$

$5.7 k[i]=1.0, R_{m}=10^{5} \frac{g}{\mathrm{~cm}^{3} \cdot s}, \bar{P}_{m}=10^{3} \frac{\mathrm{g}}{\mathrm{cm} \cdot \mathrm{s}^{2}}, h_{o}=\frac{1}{32}, \rho=1 \frac{\mathrm{g}}{\mathrm{cm}^{3}}, \mu=10^{-2} \frac{\mathrm{g}}{\mathrm{cm} \cdot \mathrm{s}}$, $k=9, N=32$, Variação de área: $59.98 \%$ após $t=1.0 \mathrm{~s}, 62.46 \%$ após $t=1.5 \mathrm{~s}$, Fontes centradas em $(0.2,0.5)$, Sorvedouros centrados em $(0.55,0.1)(0.65,0.15)(0.75,0.2)$ $(0.85,0.3)(0.9,0.5)(0.85,0.7)(0.75,0.8)(0.65,0.85)(0.55,0.9) \ldots \ldots \ldots 9$

$5.8 k[i]=0.1, R_{m}=10^{5} \frac{g}{\mathrm{~cm}^{3} \cdot s}, \bar{P}_{m}=10^{3} \frac{\mathrm{g}}{\mathrm{cm} \cdot \mathrm{s}^{2}}, h_{o}=\frac{1}{32}, \rho=1 \frac{\mathrm{g}}{\mathrm{cm}^{3}}, \mu=10^{-2} \frac{\mathrm{g}}{\mathrm{cm} \cdot \mathrm{s}}$, $k=9, N=32$, Variação de área: $72.63 \%$ após $t=1.0 \mathrm{~s}, 90.88 \%$ após $t=1.5 \mathrm{~s}$, Fontes centradas em $(0.2,0.5)$, Sorvedouros centrados em $(0.55,0.1)(0.65,0.15)(0.75,0.2)$ $(0.85,0.3)(0.9,0.5)(0.85,0.7)(0.75,0.8)(0.65,0.85)(0.55,0.9) \ldots \ldots \ldots . \ldots 99$

$5.9 k[i]=0.1$ no segmento $\overline{B E}$ e $k[i]=1.0$ no segmento $\overline{D E}, R_{m}=10^{5} \frac{\mathrm{g}}{\mathrm{cm}^{3} . \mathrm{s}}, \bar{P}_{m}=$ $10^{3} \frac{g}{\mathrm{~cm} \cdot \mathrm{s}^{2}}, h_{o}=\frac{1}{32}, \rho=1 \frac{\mathrm{g}}{\mathrm{cm}^{3}}, \mu=10^{-2} \frac{\mathrm{g}}{\mathrm{cm} \cdot s}, k=9, N=32$, Variação de área: $46.39 \%$ após $t=1.0 \mathrm{~s}, 60.36 \%$ após $t=1.5 \mathrm{~s}$, Fontes centradas em $(0.2,0.5)$, Sorvedouros centrados em $(0.55,0.1)(0.65,0.15)(0.75,0.2)(0.85,0.3)(0.9,0.5)(0.85,0.7)(0.75,0.8)(0.65,0.85)$ $(0.55,0.9)$

$5.10 k^{*}[i]=\operatorname{sen}\left(e^{k[i] n \Delta t}\right)$ no segmento $\overline{B E}$ e $k^{*}[i]=(k[i] n \Delta t-10)^{2}$ no segmento $\overline{D E}$, $R_{m}=10^{5} \frac{g}{\mathrm{~cm}^{3} \cdot s}, \bar{P}_{m}=10^{3} \frac{\mathrm{g}}{\mathrm{cm} \cdot \mathrm{s}^{2}}, h_{o}=\frac{1}{32}, \rho=1 \frac{\mathrm{g}}{\mathrm{cm}^{3}}, \mu=10^{-2} \frac{\mathrm{g}}{\mathrm{cm} \cdot \mathrm{s}}, k=9, N=32$, Variação de área: $33.07 \%$ após $t=1.0 \mathrm{~s}, 37.54 \%$ após $t=1.5 \mathrm{~s}$, Fontes centradas em $(0.2,0.5)$, Sorvedouros centrados em $(0.55,0.1)(0.65,0.15)(0.75,0.2)(0.85,0.3)(0.9,0.5)$

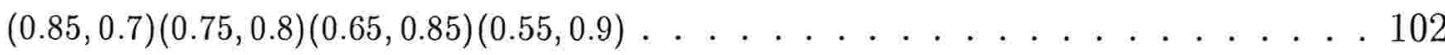

A.1 Comportamento das partículas do fluido no escoamento laminar . . . . . . . . . 105

A.2 Comportamento das partículas do fluido no escoamento turbulento . . . . . . . . . 107

A.3 Fluxo nas vias aéreas - Hopkins $[17] \ldots \ldots$. . . . . . . . . . . . . . . . 108 


\section{Lista de Tabelas}

1.1 Volumes respiratórios para homens saudáveis - Johson [18] . . . . . . . . . . . 8

1.2 Forças aplicadas-desenvolvidas pelo sistema respiratório - Mead [20] . . . . . . . . . . 10

3.1 Notação empregada $\ldots \ldots \ldots$. . . . . . . . . . . . . . . . . . . . 38

4.1 Funções para a variação temporal da elasticidade - $R_{m}=10^{5} \frac{\mathrm{g}}{\mathrm{cm}^{3} . \mathrm{s}}, \bar{P}_{m}=10^{3} \frac{\mathrm{g}}{\mathrm{cm} . \mathrm{s}^{2}}$, $h_{o}=\frac{1}{32}, \rho=1 \frac{g}{\mathrm{~cm}^{3}}, \mu=10^{-2} \frac{g}{\mathrm{~cm} . \mathrm{s}}, k=5, N=32 \ldots \ldots \ldots \ldots \ldots \ldots$

4.2 Funções para a variação temporal da elasticidade $-R_{m}=10^{5} \frac{\mathrm{g}}{\mathrm{cm}^{3} . \mathrm{s}}, \bar{P}_{m}=10^{3} \frac{\mathrm{g}}{\mathrm{cm} \cdot \mathrm{s}^{2}}$, $h_{o}=\frac{1}{32}, \rho=1 \frac{g}{c m^{3}}, \mu=10^{-2} \frac{g}{c m . s}, k=7, N=32 \ldots \ldots \ldots \ldots$

4.3 Funções para a variação temporal da elasticidade $-R_{m}=10^{5} \frac{\mathrm{g}}{\mathrm{cm}^{3} \cdot \mathrm{s}}, \bar{P}_{m}=10^{3} \frac{\mathrm{g}}{\mathrm{cm} \cdot \mathrm{s}^{2}}$, $h_{o}=\frac{1}{32}, \rho=1 \frac{g}{\mathrm{~cm}^{3}}, \mu=10^{-2} \frac{\mathrm{g}}{\mathrm{cm} . \mathrm{s}}, k=9, N=32 \ldots \ldots \ldots$. . . . . 80

A.1 Viscosidade do ar-Sears $[35] \ldots \ldots \ldots \ldots$. . . . . . . . . . . . . . . .

B.1 Número de Reynolds em algumas vias aéreas humanas - Johnson [18] . . . . . . . . 110

D.1 Variação de $R_{m}$ para $\bar{P}_{m}=10^{3}, h_{o}=\frac{1}{16}$ e $\mathrm{k}_{\max }=10^{6} \ldots \ldots \ldots \ldots$

D.2 Variação de $R_{m}$ para $\bar{P}_{m}=10^{3}, h_{o}=\frac{1}{32}$ e $\mathrm{k}_{\max }=10^{6} \ldots \ldots \ldots \ldots \ldots$

D.3 Variação de $R_{m}$ para $\bar{P}_{m}=10^{3}, h_{o}=\frac{1}{64}$ e $\mathrm{k}_{\max }=10^{6} \ldots \ldots \ldots \ldots 114$

D.4 Variação de $R_{m}$ para $\bar{P}_{m}=10^{2}, h_{o}=\frac{1}{16}$ e $\mathrm{k}_{\max }=10^{6} \ldots \ldots \ldots \ldots$

D.5 Variação de $R_{m}$ para $\bar{P}_{m}=10^{2}, h_{o}=\frac{1}{32}$ e $\mathrm{k}_{\max }=10^{6} \ldots \ldots \ldots \ldots \ldots$

D.6 Variação de $R_{m}$ para $\bar{P}_{m}=10^{2}, h_{o}=\frac{1}{64}$ e $\mathrm{k}_{\max }=10^{6} \ldots \ldots \ldots \ldots$

D.7 Variação de $R_{m}$ para $\bar{P}_{m}=10, h_{o}=\frac{1}{16}$ e $\mathrm{k}_{\max }=10^{6} \ldots \ldots \ldots \ldots$

D.8 Variação de $R_{m}$ para $\bar{P}_{m}=10, h_{o}=\frac{1}{32}$ e $\mathrm{k}_{\max }=10^{6} \ldots \ldots \ldots \ldots 17$ 
D.9 Variação de $R_{m}$ para $\bar{P}_{m}=10, h_{o}=\frac{1}{64}$ e $\mathrm{k}_{\max }=10^{6} \ldots \ldots \ldots \ldots 17$

D.10 Variação de $R_{m}$ para $\bar{P}_{m}$ variando de 10 a $0, h_{o}=\frac{1}{16}$ e $\mathrm{k}_{\max }=10^{6} \ldots \ldots \ldots$

D.11 Variação de $R_{m}$ para $\bar{P}_{m}$ variando de 10 a $0, h_{o}=\frac{1}{32}$ e $k_{\max }=10^{6} \ldots \ldots \ldots$

D.12 Variação de $R_{m}$ para $\bar{P}_{m}$ variando de 10 a $0, h_{o}=\frac{1}{64}$ e $\mathrm{k}_{\max }=10^{6} \ldots \ldots \ldots$ 


\section{Introdução}

O ciclo respiratório humano envolve processos mecânicos, como o gradiente de pressões existente ao longo da árvore respiratória, e bioquímicos, como o equilíbrio ácido-base do sangue. Todo o aparato está estruturado para levar o ar rico em oxigênio até os alvéolos pulmonares, onde as trocas gasosas ocorrem.

No mecanismo respiratório, o gradiente de pressões possibilita que o fluxo de ar vença a resistência das vias aéreas e que uma quantidade suficiente de ar fresco chegue até os alvéolos, provocando a expansão da parede dos mesmos. Essa expansão não é uniforme, sendo também determinada pela complacência pulmonar e pelo surfactante, o qual estabiliza os alvéolos. Um estudo global desse mecanismo implica no conhecimento de todos os processos mecânicos e bioquímicos envolvidos, muitos dos quais são complexos e não podem portanto ser abordados em um modelo matemático simplificado.

Neste trabalho, emprega-se o método da fronteira imersa para modelar a expansão do alvéolo durante a inspiração. A variação de parâmetros fisiológicos tais como a complacência e o surfactante é introduzida no modelo computacional através de fontes e sorvedouros e de uma função $k(t)$ para a elasticidade na fronteira, ou seja, o evento respiratório é analisado de forma local. O modelo matemático adotado simplifica radicalmente o aparelho respiratório: um tubo cilíndrico representa o bronquíolo enquanto que uma esfera acoplada a uma das extremidades do tubo simboliza o alvéolo. A secção longitudinal desse conjunto compõe um modelo bidimensional, caracterizado em um domínio retangular contendo um fluido - o ar - e uma fibra elástica fechada - a superfície do bronquíolo e do alvéolo - nele imersa. O fluido nas regiões interior e exterior à fibra é o mesmo, fato este que constitui mais uma simplificação do modelo real, uma vez que nele temos dois 
fluidos distintos: ar e sangue.

No modelo bidimensional, a expansão da parede alveolar é medida pela variação de área que deve ocorrer para que se tenha a variação de volume conhecida para o alvéolo na inspiração. A variação de área é simulada pelo ajuste da flexibilidade da fibra que representa a superfície do bronquíolo e do alvéolo e pela disposição de fontes e sorvedouros no domínio de estudo da interação fluido-fibra.

A inserção de fontes e sorvedouros possibilita a injeção de fluido no domínio computacional. O fluxo desse fluido é controlado pela adequação dos valores da pressão e da resistência associadas às fontes e aos sorvedouros. Para a introdução destes, parte-se do trabalho desenvolvido por Remigio [29], o qual analisou o comportamento do método da fronteira imersa mediante a introdução de uma fonte e de um sorvedouro no domínio computacional $\Omega=[0,1] \mathrm{X}[0,1]$. Neste domínio, inseriu uma fibra elástica fechada cuja região interior continha o centro da fonte e do sorvedouro. A força exercida pelos pontos que representam a presença da fibra foi modelada com a utilização de uma constante elástica de valor elevado. Desta forma, a fronteira imersa representada pela fibra manteve-se rígida no decorrer do tempo.

No estudo da expansão da superfície alveolar, a rigidez é requerida apenas para a parte da fronteira imersa que representa o bronquíolo respiratório. Para a porção que simula a parede alveolar espera-se uma certa mobilidade, a qual é obtida e controlada com a introdução de um número adequado de fontes e sorvedouros e o posicionamento destes, bem como com o emprego de valores para a elasticidade variáveis no tempo. Com a variação temporal da elasticidade pode-se ajustar a flexibilidade da fibra para se obter expansões diferenciadas ao longo do tempo. Isto permite a alteração de condições fisiológicas, como a complacência do alvéolo, e é possível então simular expansões que representam alvéolos sadios ou não e, conseqüentemente, modelar certas doenças do aparelho respiratório.

O trabalho de modelagem da expansão alveolar está organizado em cinco capítulos. No primeiro deles, descreve-se alguns conceitos físicos e biológicos relativos ao sistema respiratório e à mecânica respiratória, relevantes à caracterização do problema e, por conseguinte, do modelo adotado.

Uma descrição do método da fronteira imersa é feita no segundo capítulo. Características, aplicabilidade e limitações do método, equações para o escoamento do fluido 
e a força exercida pela fronteira, bem como as alterações provocadas pela introdução de fontes e sorvedouros são apresentadas.

No terceiro capítulo, as equações são discretizadas e o método computacional é sintetizado.

Valores para a pressão e a resistência nas fontes e sorvedouros são testados no quarto capítulo. Nele também se analisa a comportamento da vazão associada às fontes e aos sorvedouros e a variação temporal dos valores iniciais atribuídos à elasticidade. Diferentes funções que alteram os valores da elasticidade no decorrer do tempo são testadas com o objetivo de identificar padrões representativos para a expansão do alvéolo.

As simulações correspondentes a algumas das funções testadas no quarto capítulo são apresentadas no quinto capítulo.

Finalmente, comenta-se possíveis aplicações dos resultados obtidos. 


\section{Aspectos mecânicos e fisiológicos da respiração}

O sistema respiratório tem por função manter os equilíbrios térmico e ácido-base $(\mathrm{pH})$ do organismo, fornecendo oxigênio $\left(\mathrm{O}_{2}\right)$ para o sangue e removendo do mesmo dióxido de carbono $\left(\mathrm{CO}_{2}\right)$ proveniente da respiração celular. Ele é constituído pelas vias aéreas - cavidades nasais e oral, faringe, laringe, traquéia, brônquios e bronquíolos - pulmões, caixa toráxica, diafragma e músculos intercostais.

A partir da traquéia iniciam as ramificações da árvore respiratória. Os brônquios, primeiras ramificações, dão origem aos subbrônquios, que por sua vez se subdividem em bronquíolos. Os bronquíolos continuam se subdidividindo em tubos de diâmetro cada vez menor até atingir o nível alveolar. O modelo para a árvore respiratória mais empregado é o de Weibel - Johnson [18], o qual adota 23 níveis de ramificação ou gerações, que iniciam na traquéia e terminam no alvéolo. Os 16 primeiros níveis de subdivisão caracterizam-se pela ausência de alvéolos, constitutindo a zona de condução. A partir do $17^{\circ}$ nível, os bronquíolos começam a apresentar alvéolos em suas paredes, razão pela qual são denominados bronquíolos respiratórios. A zona respiratória compreende os níveis 20 a 23 , onde se encontram os dutos e sacos alveolares - Figura 1.1.

Os alvéolos pulmonares são estruturas poliédricas - West [41] - onde ocorre a hematose, isto é, a troca de oxigênio e dióxido de carbono entre os pulmões e o sangue. Os pulmões são constituídos de 250 a 350 milhões dessas estruturas, as quais têm um diâmetro de 200 a $300 \mu m\left(1 \mu m=10^{-6} \mathrm{~m}\right)$ e perfazem uma superfície que varia de 50 

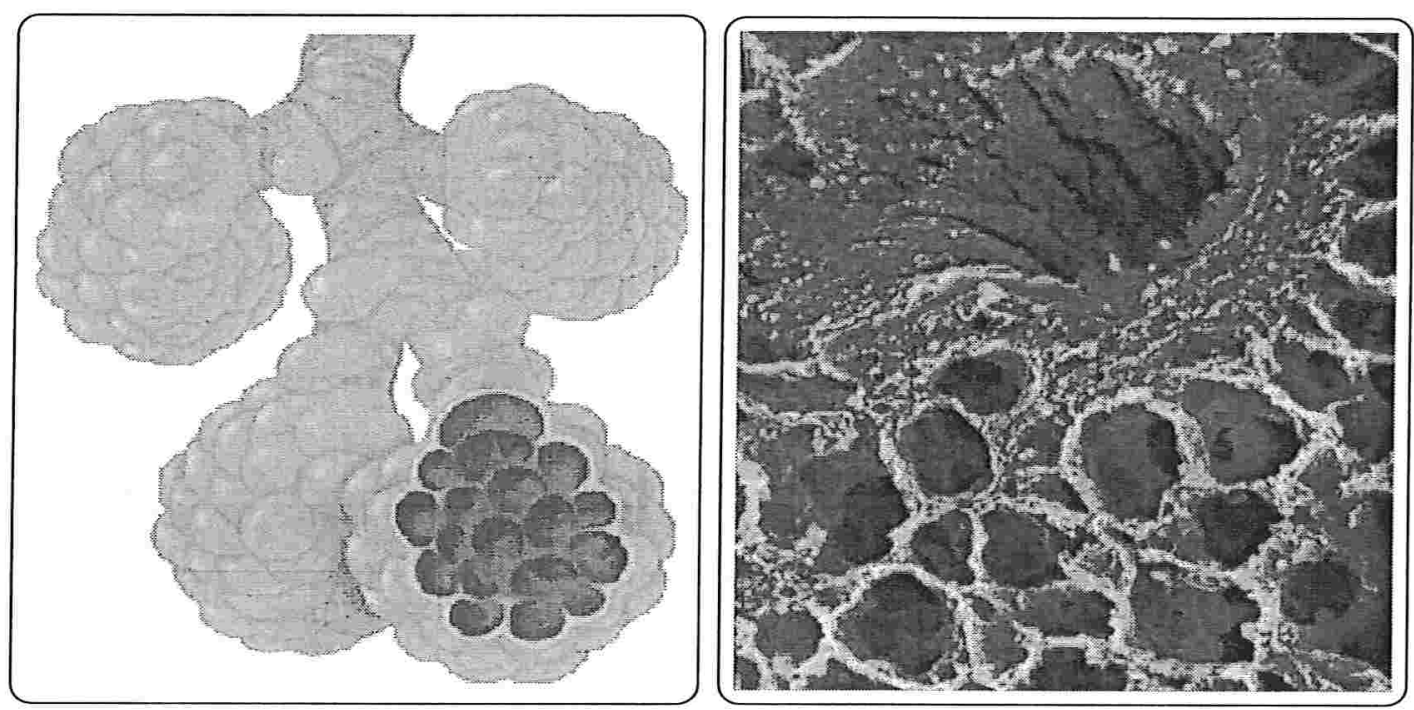

Figura 1.1: Alvéolos pulmonares - Hopkins [17], Enciclopédia Encarta [7]

a $100 \mathrm{~m}^{2}$, dependendo do grau de inflação dos pulmões. A Figura 1.2 mostra os cortes tridimensional e bidimensional de alvéolos.

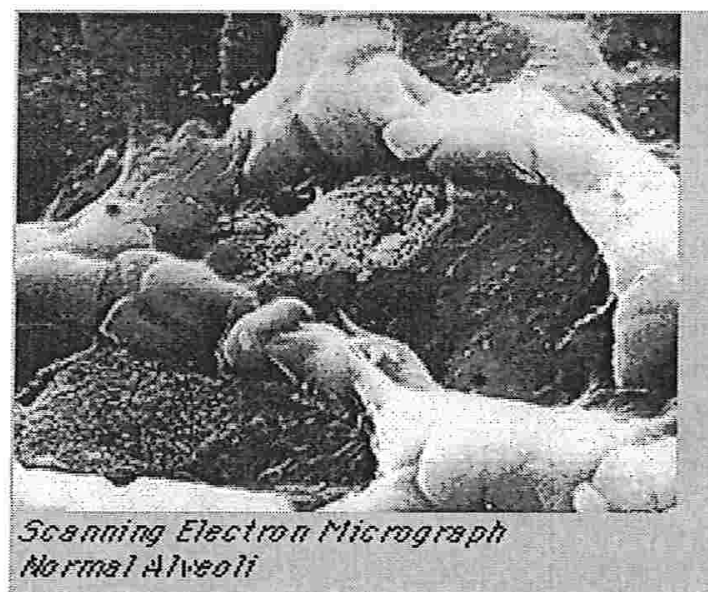

Figura 1.2: Alvéolos em corte tridimensional e bidimensional - Hopkins [17]

Existe um rico complexo capilar circundando os alvéolos. A distância que separa o espaço alveolar do sangue presente nos capilares pulmonares é da ordem de $4 \times 10^{-7} \mathrm{~m}=$ $0,0004 \mathrm{~mm}$. Essa "barreira" extremamente fina permite uma troca gasosa rápida. Na hematose, o oxigênio se difunde através da membrana alveolar, de um delgado espaço intersticial e da parede do capilar, alcançando os glóbulos vermelhos que circulam praticamente enfileirados nos capilares pulmonares; o dióxido de carbono faz o percurso inverso 
para atingir o alvéolo. A Figura 1.3 ilustra o complexo capilar que circunda os alvéolos e destaca a barreira através da qual o oxigênio e o gás carbônico se difundem na hematose.

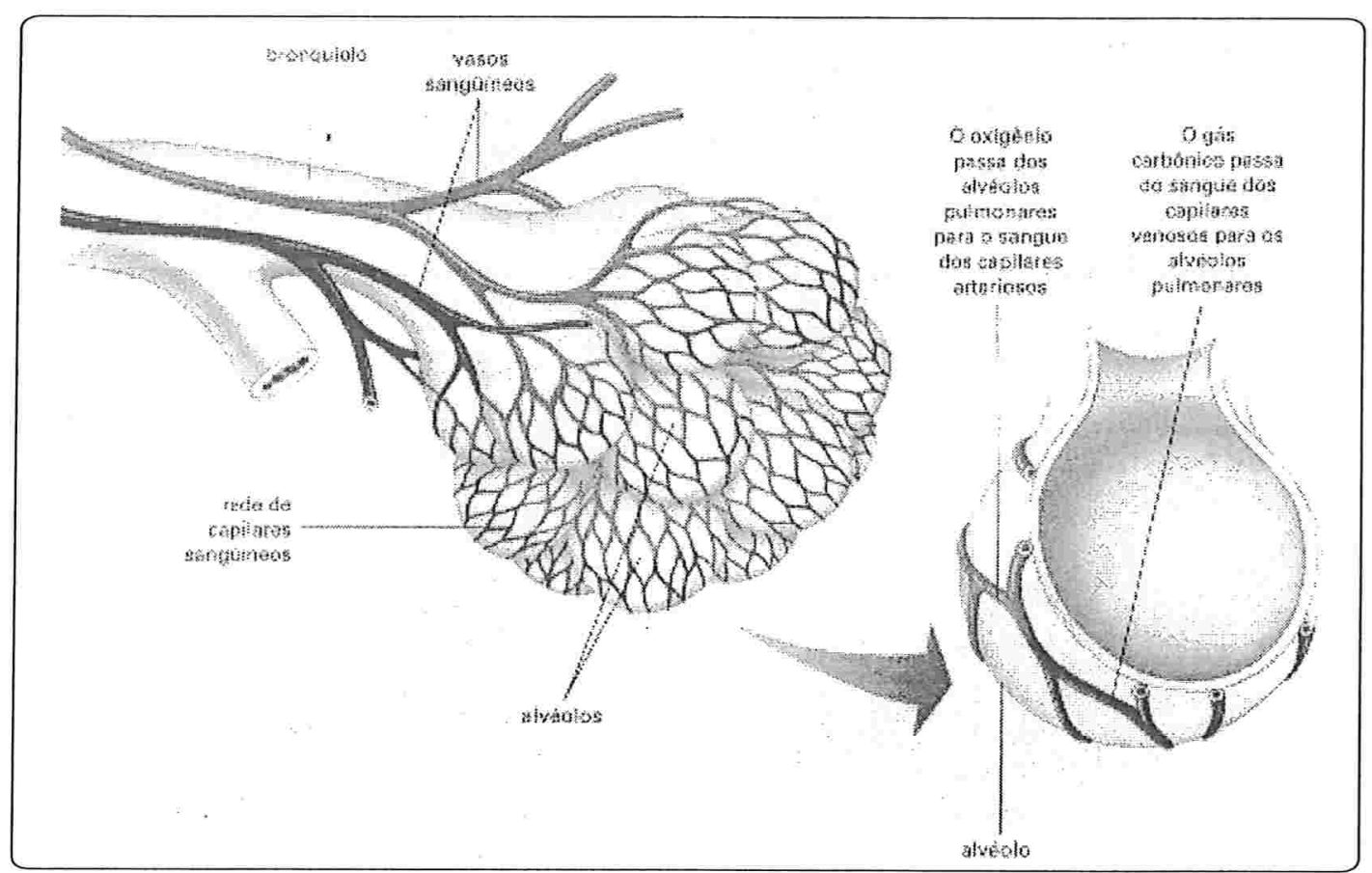

Figura 1.3: Complexo capilar que circunda os alvéolos - Corpo Humano [5]

O ciclo respiratório humano compreende dois movimentos sucessivos: a inspiração e a expiração, e dura normalmente cerca de $4 s$ para um indivíduo sadio e em repouso. Os movimentos respiratórios são efetuados devido à ação muscular do diafragma - músculo responsável por $75 \%$ da expansão da cavidade toráxica e, portanto, fundamental no desenvolvimento da pressão requerida para mover ar para os pulmões - e dos músculos intercostais, todos controlados pelo centro respiratório localizado no bulbo, um dos componentes do sistema nervoso central.

É a pressão dentro do alvéolo que determina se o ar fluirá das vias aéreas superiores para os pulmões ou vice-versa. Durante a inspiração espontânea, o diafragma se contrai ao mesmo tempo que a caixa toráxica e os pulmões se expandem. Com os pulmões expandidos, a pressão alveolar se torna negativa em relação à pressão das vias aéreas, considerada nula na boca, e o gás atinge os alvéolos. A inspiração continua até que a pressão alveolar fique positiva. O diafragma e os músculos intercostais então relaxam e a caixa toráxica e os pulmões tendem a voltar para seu tamanho e posição iniciais. 
No ciclo respiratório, os pulmões nunca ficam completamente vazios. O volume de gás que permanece nos pulmões ao final da exalação, ou capacidade residual funcional (FRC), é de aproximadamente $2,4 \ell$. Na inspiração, o volume de ar que entra nos pulmões, ou volume de ar corrente $\left(V_{T}\right)$, é de cerca de $0,4 \ell$. Contudo, esse volume inspirado não chega todo até os alvéolos; uma parte dele preenche o espaço das vias aéreas, chamado espaço morto anatômico $\left(V_{\text {Danat }}\right)$. O gás que ventila os alvéolos mas não é difundido para os capilares pulmonares durante a hematose também produz um "espaço morto", chamado espaço morto alveolar $\left(V_{D a l v}\right)$. A soma desses espaços mortos define o espaço morto fisiológico $\left(V_{D \text { phys }}\right)$. Portanto:

$$
V_{\text {Dphys }}=V_{\text {Danat }}+V_{\text {Dalv }}
$$

Em indivíduos normais

$$
V_{\text {Dphys }}=V_{\text {Danat }}
$$

uma vez que $V_{D a l v}$ é muito pequeno.

A ventilação alveolar $\left(V_{A}\right)$ é a ventilação efetiva, isto é, a quantidade de gás fresco que chega até os alvéolos. Matematicamente, pode-se expressá-la como sendo a diferença entre o volume de ar corrente e o espaço morto anatômico. Assim:

$$
V_{A}=V_{T}-V_{\text {Danat }}
$$

A Tabela 1.1 traz alguns volumes típicos para homens normais e saudáveis.

\begin{tabular}{|l|l|}
\hline VOLUMES TÍPICOS & VALORES NORMAIS \\
\hline Capacidade pulmonar total & $6,0 \times 10^{-3} \mathrm{~m}^{3}=6,0 \ell$ \\
\hline FRC & $2,4 \times 10^{-3} \mathrm{~m}^{3}=2,4 \ell$ \\
\hline $\mathrm{V}_{\text {Danat }}$ & $1,5 \times 10^{-4} \mathrm{~m}^{3}=0,15 \ell$ \\
\hline $\mathrm{V}_{\text {Dphys }}$ & $1,8 \times 10^{-4} \mathrm{~m}^{3}=0,18 \ell$ \\
\hline $\mathrm{V}_{T}$ durante repouso & $4,0 \times 10^{-4} \mathrm{~m}^{3}=0,4 \ell$ \\
\hline $\mathrm{V}_{T}$ durante exercícios físicos pesados & $2,0 \times 10^{-3} \mathrm{~m}^{3}=2,0 \ell$ \\
\hline $\mathrm{V}_{A}$ durante repouso & $2,5 \times 10^{-4} \mathrm{~m}^{3}=0,25 \ell$ \\
\hline $\mathrm{V}_{A}$ durante exercícios físicos pesados & $1,8 \times 10^{-3} \mathrm{~m}^{3}=1,8 \ell$ \\
\hline
\end{tabular}

Tabela 1.1: Volumes respiratórios para homens saudáveis - Johson [18] 
Durante a ventilação espontânea, o volume de ar corrente nunca é distribuído uniformemente através dos pulmões. Logo, os alvéolos não são ventilados todos da mesma maneira. A difusão dos gases também não ocorre uniformemente nos pulmões. Ela varia segundo um gradiente de pressão hidrostático nas veias pulmonares e com a posição do corpo (devido às forças gravitacionais), sendo sempre maior na base dos pulmões quando o corpo está na posição vertical.

Para que a hematose ocorra é necessário que um determinado volume $\Delta V$ de ar seja movimentado durante o ciclo respiratório. Isto depende de três fatores: da resistência $\mathrm{R}$ das vias aéreas, da complacência $\mathrm{C}$ dos pulmões e de uma variação de pressão $\Delta P$ nas vias aéreas produzida pelo músculo diafragma.

\subsection{Pressões}

O que existe realmente no sistema respiratório humano é um gradiente de pressões, isto é, a distribuição da pressão entre os vários ramos que compõem a árvore respiratória. No modelo matemático para a expansão do alvéolo pulmonar emprega-se um valor médio para a pressão ao invés de um gradiente de pressões. O mesmo ocorre na ventilação mecânica ou assistida, ventilação efetuada por um aparelho que auxilia o paciente a executar os movimentos respiratórios, na qual a variação de pressão é dada pela diferença entre a pressão exercida pelo ventilador e a pressão alveolar desenvolvida pelo diafragma e músculos intercostais.

Segundo Mead [20], a análise mecânica do sistema respiratório humano deve compreender a análise das três partes constitutivas do mesmo:

- o gás nas vias e espaços aéreos;

- a estrutura pulmonar - incluindo, além dos pulmões, os tecidos e vias aéreas;

- o tórax contendo os pulmões.

A pressão aplicada a essas partes é igual à diferença de pressão nas fronteiras de cada uma delas. A partir dessa afirmativa, a terceira lei de Newton pode ser expressa da seguinte forma: 


\begin{tabular}{|l|c|c|}
\hline PARTE & PRESSÃO APLICADA & PRESSÃO OPOSTA \\
\hline Gás $(G)$ & $P_{a o}-P_{a l v}$ & $P_{G}$ \\
\hline Tecido $(T i)$ & $P_{a l v}-P_{p l}$ & $P_{T i}$ \\
\hline Tórax $(W)$ & $P_{p l}-P_{b s}$ & $P_{W}$ \\
\hline
\end{tabular}

Tabela 1.2: Forças aplicadas-desenvolvidas pelo sistema respiratório - Mead [20]

onde

$P_{a \circ}$ : pressão nas vias aéreas superiores (boca)

$P_{a l v}:$ pressão alveolar

$P_{p l}:$ pressão pleural

$P_{b s}:$ pressão na superfície do corpo

Durante o ciclo respiratório assume-se que

$$
P_{a o}=P_{b s}=P_{a t}
$$

onde $P_{a t}$ representa a pressão atmosférica.

A pressão total $\left(P_{T}\right)$ no sistema respiratório é dada pela soma das pressões aplicadas a cada uma de suas partes ou desenvolvidas por elas. Assim:

$$
P_{T}=P_{G}+P_{T i}+P_{W}
$$

Substituindo-se em (1.2) as pressões fornecidas pela Tabela 1.2:

$$
P_{T}=P_{a o}-P_{a l v}+P_{a l v}-P_{p l}+P_{p l}-P_{b s}=P_{a o}-P_{b s}
$$

Associando as igualdades (1.1) e (1.3), tem-se que

$$
P_{T}=0
$$

O resultado anterior expressa o princípio de D'Alembert, o qual afirma que a soma algébrica das forças aplicadas em um corpo é zero.

A pressão pleural $P_{p l}$ permite que os pulmões acompanhem a caixa toráxica durante os movimentos respiratórios, sendo gerada por uma delgada camada de líquido existente entre 
as membranas que revestem externamente os pulmões (pleura visceral) e internamente a caixa toráxica (pleura parietal). Esse líquido lubrifica as superfícies dessas membranas e produz um fenômeno de capilaridade entre elas, semelhante àquele que ocorre quando dois pratos ficam aderidos um ao outro ao se colocar uma pequena quantidade de água entre eles.

Quanto à pressão alveolar $P_{a l v}$, o método mais direto para medi-la consiste na inserção de uma cânula dentro dos alvéolos. Embora o método tenha sido empregado em ratos, cães e gatos, ele é inadequado para humanos. Otis [22] descreve um método para obter a pressão alveolar humana e conclui que, em geral, é necessária uma pressão alveolar de $1.8 \mathrm{~cm} \mathrm{H}_{2} \mathrm{O}$ para gerar um fluxo de ar de $500 \mathrm{~m} \ell / \mathrm{s}$.

Considerando-se que o fluxo de gás gera uma variação uniforme de volume $\Delta V$ em cada uma das partes do sistema, tem-se que:

$$
\Delta V_{T}=\Delta V_{G}=\Delta V_{T i}=\Delta V_{W}
$$

A análise mecânica do sistema respiratório procura expressar a afinidade entre as expressões (1.4) e (1.2), ou seja, entre o movimento do sistema respiratório e as forças associadas a esses movimentos.

Uma vez definida a variação de pressão $\Delta P$, o ar ainda precisa vencer duas barreiras até chegar aos alvéolos: a resistência das vias aéreas e a complacência do sistema na sua expansão.

\subsection{Resistência das vias aéreas}

A resistência das vias aéreas $\left(\mathrm{R}_{A W}\right)$ é a oposição que as mesmas oferecem ao escoamento do gás, causada pelas forças de friç̧ão. Ela é definida como sendo a razão entre a variação de pressão entre a boca e o alvéolo e a vazão volumétrica de fluido

$$
\begin{gathered}
R_{A W}=\frac{\Delta P}{\dot{V}} \\
R_{A W}=\frac{P_{a o}-P_{a l v}}{\dot{V}}
\end{gathered}
$$

e medida em $\frac{\mathrm{cmH}_{2} \mathrm{O}}{\ell / s}$. 
A resistência ao fluxo nas vias aéreas depende do tipo de escoamento, se laminar ou turbulento (Apêndice A), das dimensões da via aérea e da viscosidade do gás. Na ventilação espontânea, a resistência das vias aéreas em um adulto é estimada entre 2 e $3 \frac{c m \mathrm{H}_{2} \mathrm{O}}{\ell / s}$. Já na ventilação mecânica, a estimativa é de $6 \frac{c m \mathrm{H}_{2} \mathrm{O}}{\ell / s}-$ Dupuis [6].

Para escoamentos laminares, a equação de Hagen-Poiseuille - equação (A.1) - pode ser rearranjada de modo a se obter uma expressão para o cálculo da resistência.

$$
\begin{aligned}
& \Delta P=\frac{8 \mu L}{\pi r^{4}} \dot{V} \\
& \frac{\Delta P}{\dot{V}}=\frac{8 \mu L}{\pi r^{4}}
\end{aligned}
$$

Empregando (1.5), tem-se que

$$
R=\frac{8 \mu L}{\pi r^{4}}
$$

Então, segundo a equação (1.6), quanto menor o raio do tubo por onde o fluido escoa maior será a resistência ao escoamento, isto se a viscosidade do fluido e o comprimento do tubo forem mantidos constantes.

Porém, a resistência das vias aéreas ao fluxo de gás depende também do número de caminhos paralelos (ramificações) existentes. Assim, vias aéreas de maior diâmetro, como a traquéia e os brônquios, oferecem maior resistência ao fluxo do que as vias aéreas provenientes de muitas ramificações, como os bronquíolos respiratórios.

No modelo matemático, resistência e pressão estão associadas às fontes e aos sorvedouros introduzidos no domínio computacional. A adequação dos valores da pressão e da resistência é fundamental à expansão que se deseja para a parte da fronteira imersa que simula a membrana alveolar.

\subsection{Complacência}

A elastância ou substância elástica (E) é definida como a capacidade que uma substância tem de resistir à deformação por pressão. Matematicamente, a elastância é expressa como sendo a razão entre as variações de pressão e de volume

$$
E=\frac{\Delta P}{\Delta V}
$$


e medida em $\frac{c m \mathrm{H}_{2} \mathrm{O}}{\ell}$.

A razão inversa à elastância caracteriza a complacência (C), a qual é definida como sendo a variação de volume de uma estrutura produzida por uma variação de pressão através da estrutura. Assim:

$$
C=\frac{\Delta V}{\Delta P}
$$

A complacência, medida em $\frac{\ell}{\mathrm{cmH}_{2} \mathrm{O}}$, refere-se à distendibilidade de uma estrutura elástica, como o pulmão por exemplo. Ela permite compreender que o volume de uma estrutura elástica não aumenta se as pressões dentro e em torno dela aumentarem igualmente ao mesmo tempo. Portanto, quando o pulmão aumenta em tamanho mais pressão deve ser aplicada para se obter o mesmo aumento em volume.

A complacência total $\left(C_{T}\right)$ engloba as complacências da caixa toráxica $\left(C_{C W}\right)$ e dos pulmões $\left(C_{L}\right)$ e pode ser determinada segundo a expressão

$$
\frac{1}{C_{T}}=\frac{1}{C_{C W}}+\frac{1}{C_{L}}
$$

Nos pulmões, a complacência total determina a variação de volume para cada unidade de variação de pressão.

A complacência varia com a posição do corpo, a idade e várias patologias, como a fibrose e o enfisema. Na fibrose, os pulmões tornam-se "duros" e pressões altas são necessárias para manter volumes moderados; nesse caso, os pulmões têm baixa complacência. No enfisema, os pulmões tornam-se "moles" devido ao rompimento das paredes de muitos alvéolos; nesse caso, pequenas pressões são suficientes para manter grandes volumes e, portanto, os pulmões têm alta complacência.

$\mathrm{Na}$ respiração espontânea, o valor normal para a complacência é de aproximadamente $0,1 \frac{\ell}{c m H_{2} \mathrm{O}}$. Na respiração assistida, esse valor é de $0,05 \frac{\ell}{c m H_{2} \mathrm{O}}-$ Dupuis [6].

A complacência pulmonar será modelada através da adequação dos valores da pressão e da resistência associadas às fontes e aos sorvedouros, bem como pela atribuição de valores variáveis no tempo à elasticidade da fronteira imersa. Então, dependendo dos valores atribuídos à elasticidade, à pressão e à resistência, pode-se simular expansões com menor ou maior variação de área, imitando o que ocorre com pulmões sadios ou afetados por alguma doença. 


\subsection{Surfactante - Tensão superficial}

Nos alvéolos existem dois tipos de células epiteliais: as células do tipo I e do tipo II. As células do tipo I têm longas extensões citoplasmáticas e compreendem o delgado epitélio alveolar; as células do tipo II são mais compactas e produzem o surfactante.

O surfactante é uma substância, tal como um detergente, adicionada a um líquido para aumentar as propriedades difusivas deste pela redução da tensão superficial.

A tensão superficial, medida em $\frac{N}{m}$ ou $\frac{d y n}{c m}\left(1 N=10^{5} d y n\right)$, é resultante de forças intermoleculares e faz com que a superfície de um líquido se comporte como uma membrana elástica esticada. No interior de um líquido, uma molécula sofre forças de atração das moléculas ao seu redor, enquanto que na superfície uma molécula é atraída somente pelas moléculas abaixo dela. Quando uma molécula da superfície é ligeiramente elevada, as ligações moleculares entre ela e as moléculas vizinhas são alongadas e surge então uma força restauradora que tende a colocar a molécula deslocada novamente na superfície. É essa força que, por exemplo, suporta uma agulha quando esta é colocada cuidadosamente na superfície da água - Sears [35].

A tensão superficial da película de surfactante presente nos alvéolos pulmonares varia geralmente entre 25 e $30 \frac{d y n}{c m}$ - Smith [36], isto quando o corpo está em repouso.

Nos pulmões, alvéolos e bronquíolos menores são revestidos internamente por uma fina camada de surfactante, sem a qual alvéolos maiores tenderiam a aumentar e pequenos alvéolos colapsariam. A Figura 1.4 ilustra comparativamente alvéolos revestidos e não revestidos internamente por uma camada de surfactante, destacando no canto superior direito as células responsáveis pela produção dessa substância. O surfactante reduz a tensão superficial por todo pulmão, contribuindo para sua complacência total e estabilizando o alvéolo.

O valor da tensão superficial influencia na pressão exercida por um gás contido em uma estrutura esférica. A lei de Laplace diz que a pressão dentro de uma estrutura esférica com tensão superficial é inversamente proporcional ao raio da esfera. Assim, para uma esfera com duas interfaces líquido-gás, como uma bolha de sabão, a pressão é dada por

$$
P=\frac{4 \gamma \Delta r}{r}
$$




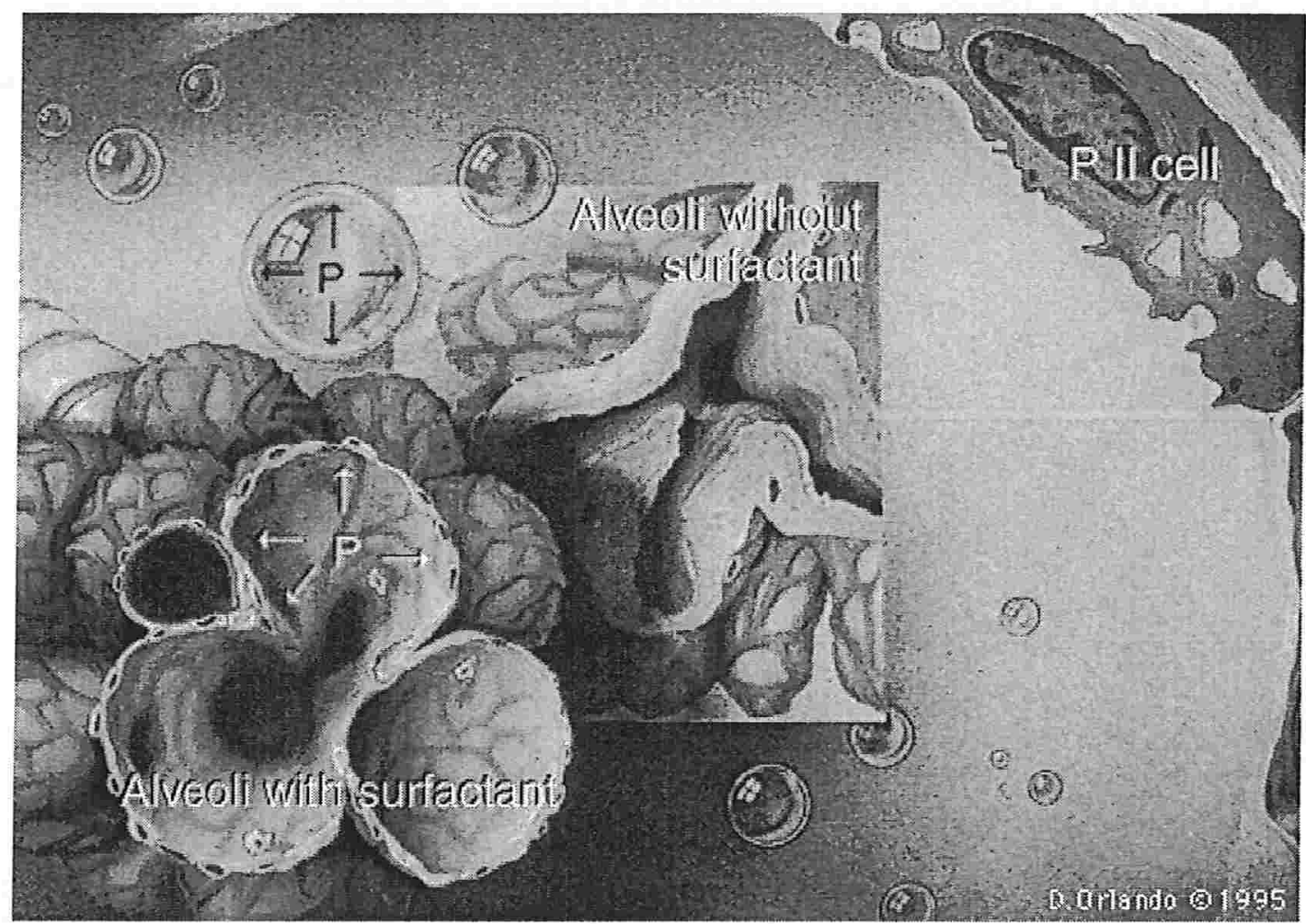

Figura 1.4: Contribuição do surfactante à estabilidade do alvéolo - Hopkins [17]

onde

$r$ : raio da esfera

$\gamma:$ tensão superficial

$\Delta r:$ espessura da parede da esfera

Já para uma esfera com uma interface líquido-gás, como um alvéolo pulmonar, a pressão é fornecida por

$$
P=\frac{2 \gamma \Delta r}{r}
$$

Pela equação (1.7), pequenos alvéolos têm, a uma tensão superficial constante, pressões internas maiores do que grandes alvéolos. Então, pequenos alvéolos deveriam se "esvaziar" dentro de alvéolos maiores quando o volume pulmonar diminui e o pulmão seria visto como um grande e expandido alvéolo acompanhado de inúmeros pequenos alvéolos colapsados. Porém isto não ocorre porque o surfactante reduz diferencialmente a tensão superficial, mais em pequenos volumes e menos em volumes maiores, estabilizando o alvéolo e evitando seu colapsamento. 
No modelo, a presença do surfactante também é definida pela variação temporal da elasticidade da fronteira imersa, uma vez que o mesmo altera a complacência pulmonar. 


\section{Modelo matemático}

O modelo matemático a ser empregado para o alvéolo pulmonar consiste na expansão de uma bolha na extremidade de um capilar.

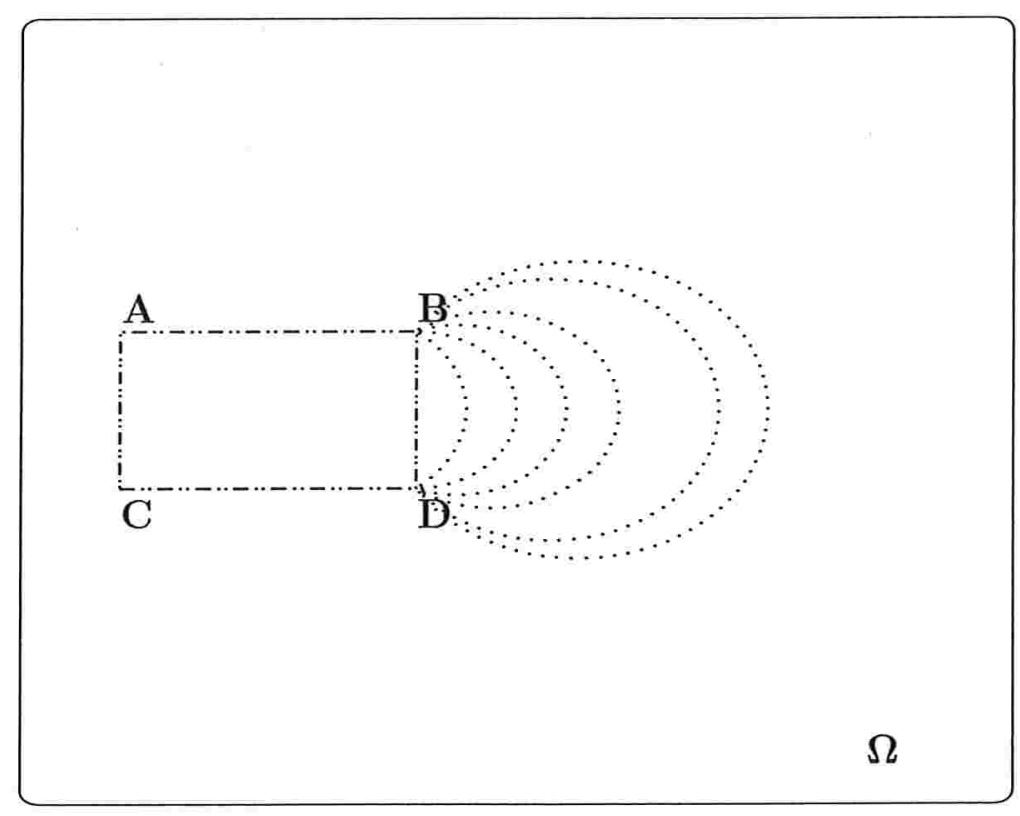

Figura 2.1: Modelo matemático para o alvéolo pulmonar

No modelo matemático bidimensional adotado para a expansão de um alvéolo - Figura 2.1, o bronquíolo respiratório e o alvéolo pulmonar são representados por uma fibra fechada completamente imersa em um fluido, nesse caso o ar. Essa fibra tem configuração inicial dada pelo retângulo $A B C D$, sendo que este representa a secção transversal de um tubo cilíndrico que simboliza o bronquíolo respiratório, enquanto que a expansão do segmento 
$\overline{B D}$ representa a secção transversal de uma esfera que simboliza o alvéolo pulmonar. Além de reduzir o aparato respiratório a um único compartimento, o modelo matemático apresenta outras simplificações, entre as quais o fato de considerar o alvéolo esférico e as paredes do bronquíolo respiratório rígidas, assim como empregar um único valor para a densidade e a viscosidade do fluido em todo o domínio de integração das equações que regem o escoamento.

Em um domínio computacional $\Omega=[0,1] \mathrm{X}[0,1]$, considerou-se por uma questão de escala um tubo de $3 \mathrm{~mm}$ de comprimento e $1 \mathrm{~mm}$ de raio. Estas medidas são um pouco elevadas em relação ao modelo real, porém permitem uma melhor vizualização das simulações. Para os vértices do retângulo tomou-se $A(0.1,0.6), B(0.4,0.6), C(0.1,0.4)$ e $D(0.4,0.4)$, o que deixa espaço suficiente no domínio computacional para a expansão do segmento $\overline{B D}$. A geometria inicial adotada é específica para o que se pretende simular. Translações ou rotações da mesma não são consideradas, uma vez que podem alterar significativamente a estrutura do modelo e os resultados esperados.

O fluxo do fluido e o comportamento dos pontos que caracterizam a fibra imersa são definidos no método da fronteira imersa pela introdução de fontes $F$ e sorvedouros $S$ no domínio computacional - Figura 2.2, e pela variação temporal da elasticidade associada aos pontos do segmento $\overline{B D}$.

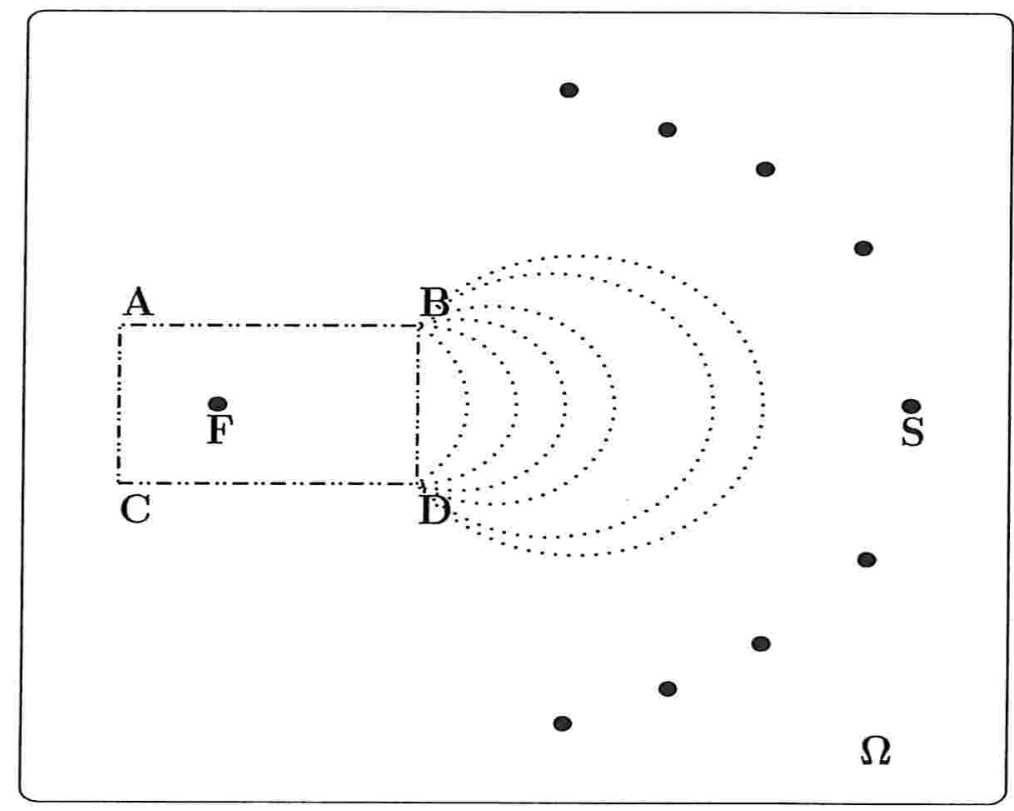

Figura 2.2: Modelo matemático com fontes e sorvedouros 
Como o modelo adotado é bidimensional, far-se-á o controle da distensão do segmento $\overline{B D}$ pela variação de área que a configuração inicial da fronteira imersa sofre ao longo do tempo. Assim, é necessário conhecer a variação de volume do alvéolo pulmonar durante a inspiração para poder estimar a variação de área.

\subsection{Variação volumétrica do alvéolo na inspiração}

O movimento de inspiração em um indivíduo sadio dura cerca de $1.5 \mathrm{~s}$ quando o corpo está em repouso. Com relação a variação volumétrica do alvéolo durante esse movimento sabe-se que - Mead [20]:

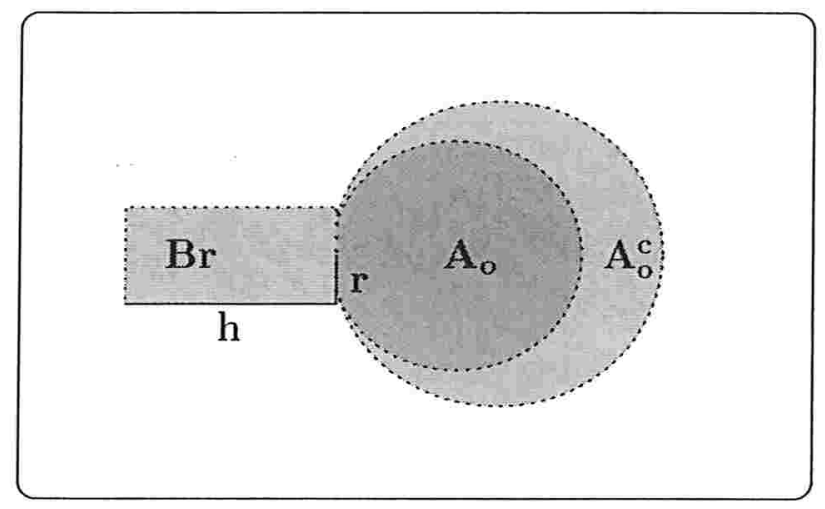

Figura 2.3: Volumes associados ao modelo

$$
\begin{aligned}
& V_{T}=V_{A}+V_{B r} \\
& V_{A}=V_{A_{\circ}}+V_{A_{o}^{c}} \\
& V_{A}=0.7 V_{T}=\frac{7}{3} V_{B r} \\
& V_{B r}=0.3 V_{T} \\
& V_{A_{o}}=0.7 V_{A}=0.7\left(0.7 V_{T}\right)=0.49 V_{T}=\frac{49}{30} V_{B r} \\
& V_{A_{o}^{c}}=0.3 V_{A}=0.3\left(0.7 V_{T}\right)=0.21 V_{T}
\end{aligned}
$$

onde

$V_{A}$ : volume do alvéolo

$V_{B r}$ : volume do bronquíolo respiratório

$V_{T}$ : volume total 
$V_{A_{o}}:$ volume da abertura alveolar - "explosão" inicial - após $\mathrm{t}=1.0 \mathrm{~s}$

$V_{A_{o}^{c}}$ : volume alveolar complementar - após $\mathrm{t}=1.5 \mathrm{~s}\left(0.5 \mathrm{~s}\right.$ após $V_{A_{\mathrm{o}}}$ ter sido atingido)

No primeiro estágio da inspiração $\left(V_{A_{o}}\right)$, o alvéolo se expande quase que imediatamente (pop up) quando a pressão alveolar atinge um valor crítico, o qual depende das condições fisiológicas do alvéolo. No segundo estágio $\left(V_{A}=V_{A_{o}}+V_{A_{o}^{c}}\right)$, durante a fase final da inspiração, o alvéolo continua a ser preenchido de ar até atingir o volume máximo, o que acontece quando a pressão alveolar se iguala a pressão nas vias aéreas.

No modelo são conhecidos o comprimento $h$ e o raio $r$ do tubo que representa o bronquíolo respiratório. Assim, o raio do alvéolo expandido nos dois estágios da inspiração é expresso em função das medidas $h$ e $r$ como:

$$
\begin{aligned}
& r_{a l v A_{o}}=\sqrt[3]{\frac{49}{40} r^{2} h} \\
& r_{a l v A}=\sqrt[3]{\frac{7}{4} r^{2} h}
\end{aligned}
$$

Considerando as medidas adotadas no modelo, isto é, $h=3 \mathrm{~mm}$ e $r=1 \mathrm{~mm}$, pode-se determinar a variação de área $\Delta A$ ocorrida nos dois estágios. Assim:

1ํㅡㄹ estágio

$$
\begin{aligned}
& A_{B_{r}}-100 \% \\
& A_{A_{o}}-x
\end{aligned}
$$

$$
\Delta A \approx 124.69 \%
$$

$2^{\text {o }}$ estágio

$$
\begin{gathered}
A_{B_{r}}-100 \% \\
A_{A}-x \\
\Delta A \approx 158.16 \%
\end{gathered}
$$

Como o alvéolo não é esférico, os cálculos anteriores constituem apenas uma estimativa a ser empregada no método da fronteira imersa para controlar a variação de área provocada pela expansão do segmento $\overline{B D}$. 


\section{$2.2 \quad \mathrm{O}$ método da fronteira imersa}

O método da fronteira imersa soluciona as equações de Navier-Stokes para um fluido incompressível de densidade e viscosidade constantes, com um termo forçante $F$ proveniente da fronteira acrescentado à equação de conservação de momento. A fronteira imersa exerce sobre as partículas do fluido vizinhas a ela uma força de densidade grande e o movimento dessas partículas induz o movimento dos pontos que representam a fronteira, acoplando assim fluido e fronteira. A integração das equações do fluido é executada em uma malha computacional Euleriana que discretiza um domínio espacial periódico. Os pontos da fronteira imersa são "perseguidos" em seu movimento juntamente com o fluido pelo emprego de uma representação Lagrangeana. A cada passo no tempo, o método utiliza a configuração da fronteira para computar as forças na fronteira, que são aplicadas aos pontos do fluido próximos à mesma. Como o fluido é incompressível, ele espalha imediatamente os efeitos da força exercida pela fronteira a todo domínio do fluxo através da ação do campo de pressões. O novo campo de velocidades do fluido é então usado para atualizar a posição dos pontos da fronteira, completando assim o acoplamento.

O método da fronteira imersa tem sido empregado na modelagem de inúmeros problemas complexos. Peskin [23] [24] [25] empregou-o no estudo do fluxo sangüíneo em torno das válvulas cardíacas, especificamente a válvula mitral, a qual controla a passagem do sangue do átrio esquerdo para o ventrículo esquerdo; Arthurs [1] no fluxo sangüíneo em uma arteríola e o respectivo transporte de massa; Fogelson [12] na agregação de plaquetas durante o processo de coagulação sangüínea; Fauci [9] [10] [11] na locomoção de animais aquáticos e na motilidade do espermatozóide; Rosar [33] no escoamento de um fluido incompressível em tubos tridimensionais colapsáveis; Ming-Chih [21] na simulação de um fluido incompressível escoando por um arranjo de cilindros circulares.

Apesar da aplicabilidade, o método da fronteira imersa tem limitações quanto à estabilidade, relacionadas principalmente à "rigidez" e à complexidade da geometria da fronteira imersa e ao emprego de números de Reynolds (Apêndice B) fisiológicos.

As limitações relativas à estabilidade podem ser contornadas com a utilização de um esquema implícito na resolução das equações de Navier-Stokes. Peskin [39] mostrou que esse esquema é incondicionalmente estável, porém muito caro computacionalmente. O uso de um esquema explícito, como o aqui empregado, exige a adoção de um passo temporal 
$\Delta t$ muito pequeno se a fronteira imersa for pouco elástica.

Neste trabalho, a geometria inicial utilizada para a fronteira imersa é relativamente simples. Entretanto, a fronteira é modelada para assumir diferentes formas no decorrer do tempo, o que se obtém através da alteração do grau de rigidez da mesma. Isto pode gerar instabilidade para certas escolhas dos parâmetros presentes no método.

Na resolução das equações de Navier-Stokes para números de Reynolds fisiológicos, a instabilidade pode ser evitada através do emprego de malhas computacionais mais refinadas, isto é, malhas com passo espacial $h$ pequeno. Porém, o uso de tais malhas aumenta consideravelmente o custo computacional. Isto pode ser amenizado pela utilização de malhas adaptativas, como a estruturada por Roma [31], as quais refinam apenas regiões de interesse no domínio computacional.

\subsubsection{Equações}

As equações de Navier-Stokes constituem um sistema acoplado de equações diferenciais parciais não-lineares que modelam o escoamento de fluidos compressíveis ou incompressíveis, laminares ou turbulentos. Formuladas com base na mecânica clássica e na termodinâmica, satisfazem os princípios de conservação de quantidade de momento, massa e energia. No método da fronteira imersa, essas equações simulam o escoamento nãoestacionário de um fluido viscoso, incompressível e de densidade constante, no qual não são considerados os efeitos da gravidade, e têm a forma

$$
\begin{aligned}
& \rho\left(\frac{\partial u}{\partial t}+u . \nabla u\right)=-\nabla p+\mu \Delta u+F \\
& \nabla . u=0
\end{aligned}
$$

onde

$\mathrm{u}=u(x, t)$ : função vetorial que representa o campo de velocidades do fluido, sendo $\mathrm{x}$ a posição espacial e t o tempo

$\mathrm{p}=p(x, t)$ : função escalar que representa o campo de pressões

$\rho$ : densidade de massa do fluido, constante no espaço e no tempo

$\mu$ : coeficiente de viscosidade do fluido, constante no espaço e no tempo

$\nabla p$ : gradiente do campo de pressões 
$\nabla u$ : gradiente do campo de velocidades

$\Delta u=\nabla^{2} u=\frac{\partial^{2} u(x, t)}{\partial x^{2}}+\frac{\partial^{2} u(x, t)}{\partial y^{2}}$ : Laplaciano do campo de velocidades

$\nabla . u$ : divergente do campo de velocidades

$\frac{\partial u}{\partial t}(x, t)+u(x, t) \cdot \nabla u(x, t)$ : derivada material, a qual fornece o valor da derivada da velocidade $\mathrm{u}$ no instante $\mathrm{t}$ ao longo da trajetória de uma partícula que nesse instante ocupa a posição $x \in \Omega$

$F$ : força singular ou a força exercida pela fronteira imersa. Esta força é descontínua, diferindo de zero somente nos pontos que representam a fronteira imersa.

A equação (2.3) representa a conservação do momento enquanto que a equação (2.4) é a equação de continuidade. O divergente do campo de velocidades nulo significa que o fluido que escoa é incompressivel. Às equações (2.3) e (2.4) são adicionadas condições de fronteira e condições iniciais para o campo de velocidades $u(x, t)$. Adota-se aqui o domínio $\Omega=[0,1] X[0,1]$ e condições periódicas para a velocidade na fronteira. Dessa forma

$$
\begin{array}{ll}
u((0, y), t)=u((1, y), t) & \forall t \geq 0 \\
u((x, 0), t)=u((x, 1), t) & \forall t \geq 0
\end{array}
$$

sendo $u(x, 0)=u_{o}(x)$, com $x \in \Omega$ e $u_{o}(x)$ fornecido, a condição inicial para a velocidade.

A função que descreve a posição dos pontos que constituem a fronteira imersa é dada por

$$
X(s, t)=\left(X_{1}(s, t), X_{2}(s, t)\right)
$$

com $s \in S=[a, b]$, onde $S$ corresponde ao comprimento total da fibra imersa.

No método da fronteira imersa, as variáveis têm uma representação mista, como ilustra a Figura 2.4. As variáveis relacionadas ao fluido - velocidade $\mathrm{u}$, pressão $\mathrm{p}$, densidade de força $\mathbf{F}$ - são descritas na forma Euleriana, pois no decorrer do tempo o comportamento das mesmas é analisado em cada ponto do domínio periódico $\Omega \subset \Re^{2}$. A posição $\mathbf{X}$ dos pontos da fibra é descrita na forma Lagrangeana, isto é, cada ponto material da fibra é identificado por um parâmetro s que controla a posição do mesmo ao longo do tempo.

Os dois componentes do esquema Euleriano-Lagrangeano são articulados por uma versão suave do delta de Dirac, o qual é usado para interpolar forças e velocidades entre partículas (pontos) do fluido e da fibra elástica imersa. O delta de Dirac permite distribuir a força exercida pela fibra às partículas do fluido e também calcular a velocidade 


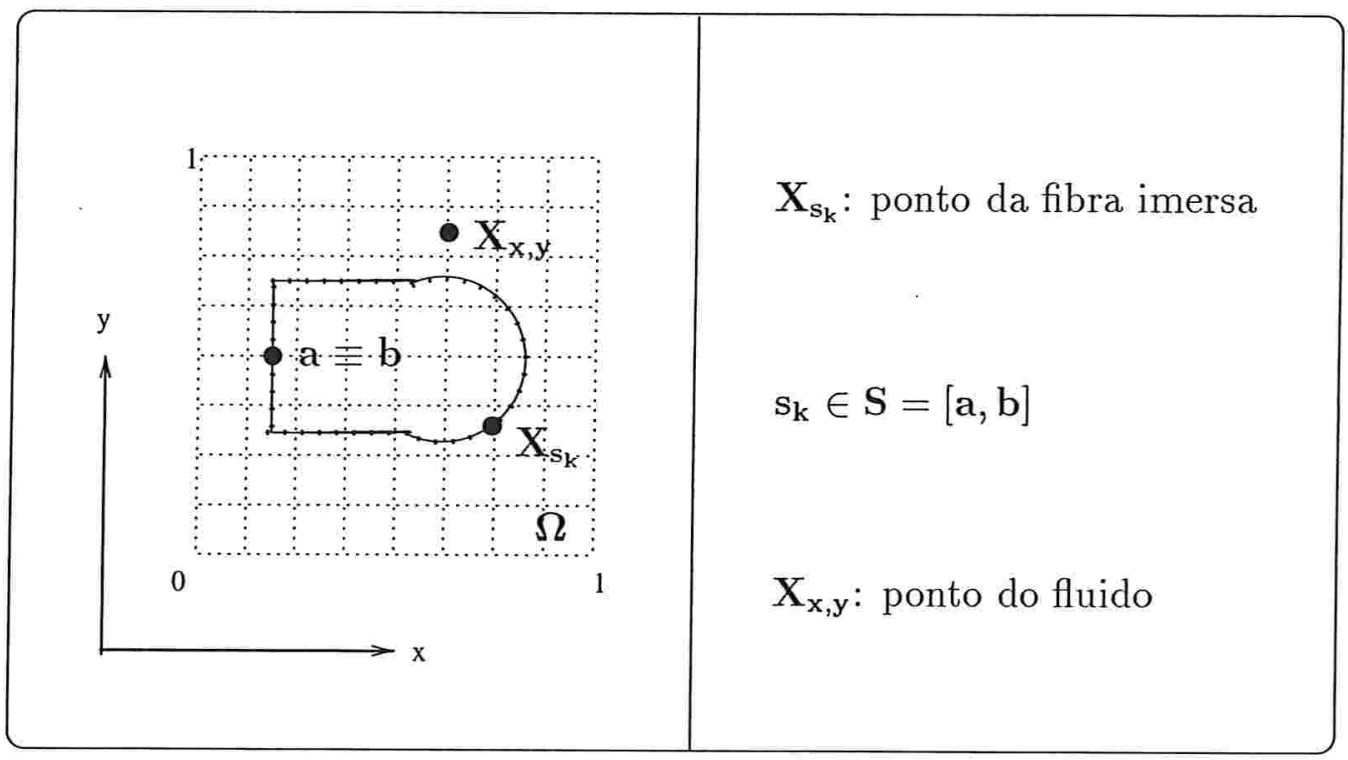

Figura 2.4: Formulação Euleriana-Lagrangeana do método da fronteira imersa

de deslocamento das partículas da fibra a partir da velocidade das partículas do fluido vizinhas a elas.

Nesse sistema, nenhuma massa é introduzida porque a fibra não ocupa volume, isto devido à suposição de que a mesma é suficientemente fina e de massa desprezível. Assim, o sistema fluido-fibra representado esquematicamente na figura acima pode ser considerado simplesmente como sendo um fluido viscoso e incompressível no qual age uma força proveniente dos pontos que representam a presença da fronteira imersa.

Considere-se agora $f(s, t)$ como sendo a densidade de força elástica exercida pela fibra. A Figura 2.5 mostra quais são as forças que atuam em um trecho arbitrário da fibra imersa.

Aplicando-se a segunda lei de Newton ao trecho arbitrário da fibra definido em $s \in$ $\left[s_{1}, s_{2}\right]$ obtém-se

$$
\frac{d}{d t} \int_{s_{1}}^{s_{2}} m(s) \frac{\partial X(s, t)}{\partial s} d s=\left.T \tau(s, t)\right|_{s_{1}} ^{s_{2}}+\int_{s_{1}}^{s_{2}}(-f(s, t)) d s
$$

onde $m(s)$ é a densidade de massa unidimensional, $T$ é a tensão na fibra, $\tau$ é o vetor tangente unitário e $(-f)$ é a força de reação do fluido à ação da fibra sobre ele.

A tensão na fibra é fornecida pela lei generalizada de Hooke

$$
T(s, t)=T_{o}\left\|\frac{\partial X}{\partial s}(s, t)\right\|
$$




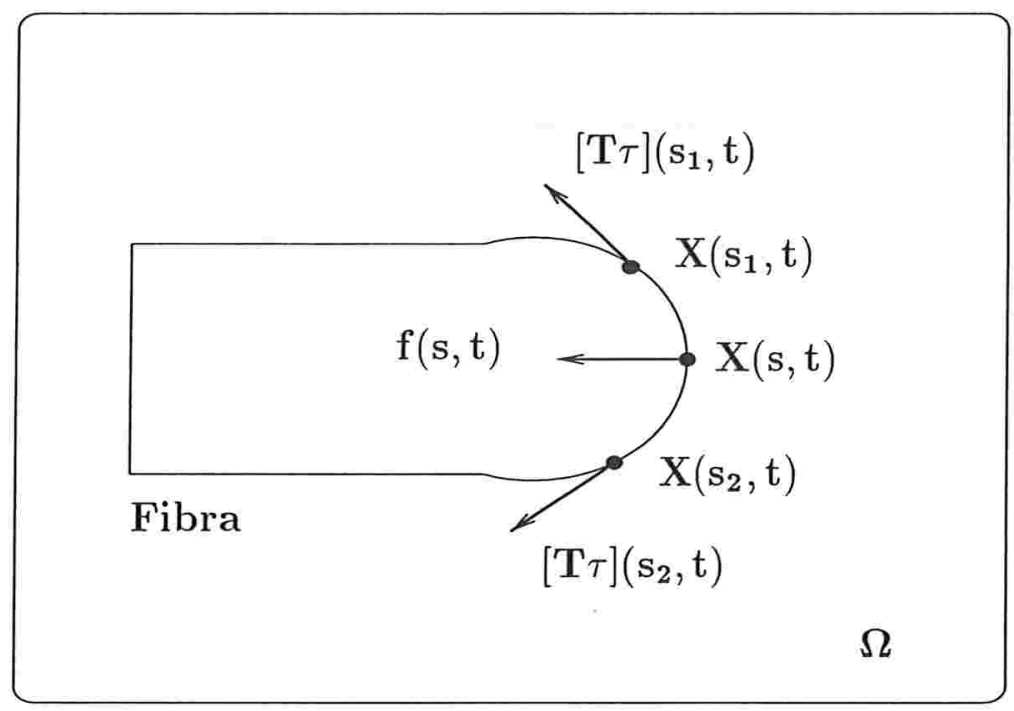

Figura 2.5: Forças atuantes em um trecho arbitrário da fibra

onde $T_{o}$ é uma constante e o vetor tangente unitário $\tau$ é dado por

$$
\tau(s, t)=\frac{\partial X(s, t) / \partial s}{\|\partial X(s, t) / \partial s\|}
$$

Como a fibra tem massa desprezível

$$
\frac{d}{d t} \int_{s_{1}}^{s_{2}} m(s) \frac{\partial X(s, t)}{\partial s} d s=0
$$

e a equação (2.5) pode ser então escrita da seguinte forma:

$$
\int_{s_{1}}^{s_{2}} f(s, t) d s=\left.T \tau(s, t)\right|_{s_{1}} ^{s_{2}}
$$

Aplicando-se o teorema fundamental do cálculo à equação (2.7), tem-se que

$$
\begin{aligned}
& \int_{s_{1}}^{s_{2}} f(s, t) d s=\int_{s_{1}}^{s_{2}} \frac{\partial T \tau}{\partial s}(s, t) d s \\
& \int_{s_{1}}^{s_{2}}\left[f-\frac{\partial T \tau}{\partial s}\right](s, t) d s=0
\end{aligned}
$$

Uma vez que $s_{1}$ e $s_{2}$ são arbitrários, pode-se concluir que a densidade de força elástica é dada por

$$
f(s, t)=\frac{\partial T \tau}{\partial s}(s, t)
$$


Desenvolvendo-se a derivada do produto na equação (2.8) chega-se a

$$
\begin{aligned}
f(s, t) & =\frac{\partial T}{\partial s} \tau+T \frac{\partial \tau}{\partial s} \\
f(s, t) & =\frac{\partial T}{\partial s} \tau+T\left\|\frac{\partial X}{\partial s}\right\| K n
\end{aligned}
$$

onde $K=\left\|\frac{\partial \tau}{\partial s}\right\| /\left\|\frac{\partial X}{\partial s}\right\|$ é a curvatura da fibra e $n=\frac{\partial \tau}{\partial s} /\left\|\frac{\partial \tau}{\partial s}\right\|$ é o vetor normal à fibra.

A densidade de força elástica representada pela equação (2.8) está expressa em função do parâmetro Lagrangeano s. Contudo, a equação do fluido (2.3) está escrita em coordenadas Eulerianas. Torna-se então necessário passar de uma formulação à outra. Para tal, considere-se uma região arbitrária $R$ do domínio $\Omega$, como mostra a Figura 2.6.

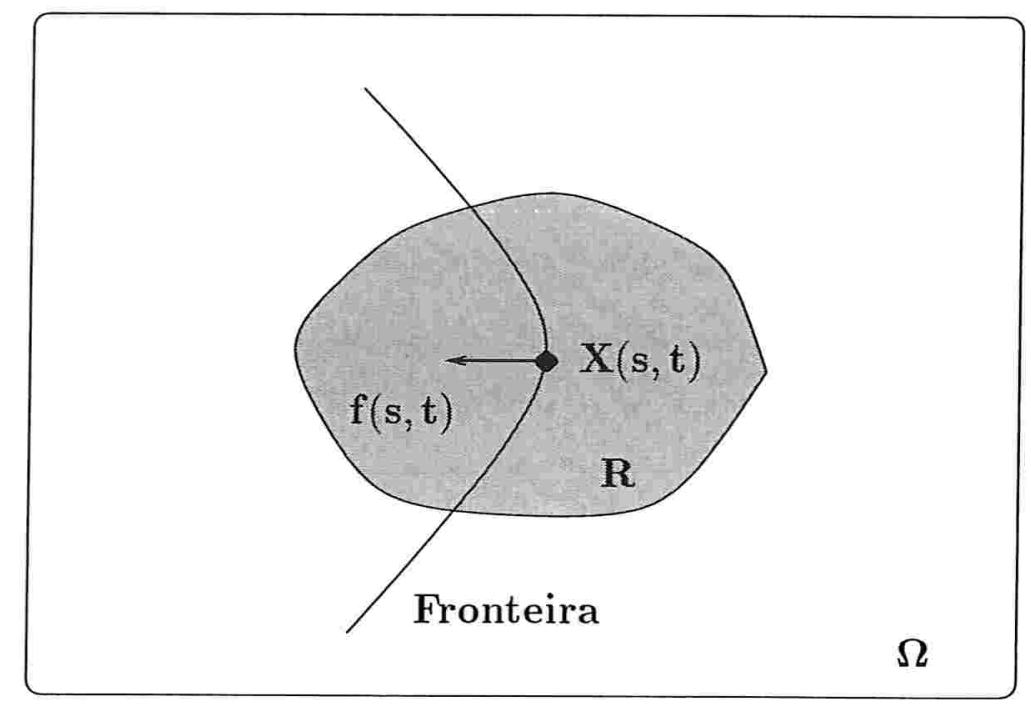

Figura 2.6: Força elástica em uma região arbitrária $\mathrm{R}$

A força total na região $R$ é fornecida por:

$$
\begin{aligned}
& \int_{R} F(x, t) d x=\int_{\{s \in S: X(s, t) \in R\}} f(s, t) d s \\
& \int_{R} F(x, t) d x=\int_{S} f(s, t) W_{R}(X(s, t)) d s \\
& \int_{R} F(x, t) d x=\int_{S} f(s, t)\left(\int_{R} \delta(x-X(s, t)) d x\right) d s \\
& \int_{R} F(x, t) d x=\int_{R}\left(\int_{S} f(s, t) \delta(x-X(s, t)) d s\right) d x
\end{aligned}
$$

onde $W_{R}(x)=\left\{\begin{array}{ll}1, & \text { se } x \in R \\ 0, & \text { caso contrário }\end{array}\right.$ e $\delta$ é o delta de Dirac bidimensional. 
Como $R$ é arbitrária, então

$$
F(x, t)=\int_{S} f(s, t) \delta(x-X(s, t)) d s
$$

A equação (2.9) expressa, em coordenadas Eulerianas, a densidade de força aplicada ao fluido pela fronteira elástica imersa.

Devido à viscosidade, os pontos da fibra movimentam-se com a velocidade local do fluido. Esta condição, conhecida como condição no-slip, pode ser matematicamente expressa em termos do campo de velocidades do fluido. Assim:

$$
\frac{\partial X}{\partial t}(s, t)=u(X(s, t), t)
$$

Novamente uma correlação entre as formulações Euleriana e Lagrangeana faz-se necessária. Empregando-se as propriedades do delta de Dirac, pode-se expressar o lado direito da equação (2.10) em função do campo de velocidades do fluido, ou seja

$$
u(X(s, t), t)=\int_{\Omega} u(x, t) \delta(x-X(s, t)) d x
$$

Substituindo (2.11) em (2.10), obtém-se

$$
\frac{\partial X}{\partial t}(s, t)=\int_{\Omega} u(x, t) \delta(x-X(s, t)) d x
$$

A equação (2.12) fornece a velocidade para os pontos da fibra a partir do campo de velocidades do fluido, permitindo a atualização da posição dos mesmos e completando assim um passo no tempo.

Com isso pode-se sintetizar o método da fronteira imersa em um conjunto de cinco equações:

$$
\begin{aligned}
& \frac{\partial u}{\partial t}+\frac{1}{\rho} \nabla p=\frac{\mu}{\rho} \Delta u-u \cdot \nabla u+\frac{1}{\rho} F \\
& \nabla \cdot u=0
\end{aligned}
$$

onde

$$
\begin{aligned}
& F(x, t)=\int_{S} f(s, t) \delta(x-X(s, t)) d s \\
& f(s, t)=\frac{\partial T \tau}{\partial s}(s, t)
\end{aligned}
$$


com

$$
\frac{\partial X}{\partial t}(s, t)=\int_{\Omega} u(x, t) \delta(x-X(s, t)) d x
$$

As incógnitas são o campo de velocidades $u=(u, v)$ e a posição $X$ da fibra. As equações (2.13) e (2.14) são as equações de Navier-Stokes para um fluido viscoso, incompressível, de densidade constante, afetado pela força exercida por um fibra elástica nele imersa. As equações (2.15) e (2.17) descrevem a interação existente entre o fluido e a fibra empregando o delta de Dirac para passar da formulação Lagrangeana para a Euleriana e vice-versa. A equação (2.16) é a equação da fronteira, a qual estabelece uma relação entre a densidade de força elástica e a tensão que atua na fronteira imersa.

A entrada e a saída de fluido em uma região do domínio computacional é modelada no método da fronteira imersa através da introdução de fontes e sorvedouros. A cada fonte é associado um sorvedouro, o qual remove o fluido fornecido pela fonte. A vazão uniforme na fonte e no sorvedouro garante a conservação de massa do fluido e a não violação da condição de incompressibilidade no domínio de integração.

Peskin [25] introduziu uma fonte e um sorvedouro em seu modelo para simular o fluxo do sangue no lado esquerdo do coração durante a diástole ventricular e o início da sístole. Arthurs [1] introduziu fontes e sorvedouros em um modelo para o fluxo de sangue e transporte de massa em uma arteríola. Remigio [29] avaliou a introdução de uma fonte e de um sorvedouro em escoamentos incompressíveis bidimensionais.

Neste trabalho, fontes e sorvedouros são introduzidos no domínio computacional com o intuito de provocar a expansão requerida para a fronteira imersa. Faz-se então uma análise detalhada do posicionamento e do número total de fontes e sorvedouros necessários à modelagem da expansão da parede alveolar.

\subsubsection{Introdução de fontes e sorvedouros}

Ao se introduzir $k$ fontes e $k$ sorvedouros no método da fronteira imersa, as equações que regem o escoamento do fluido (2.3) e (2.4) passam a ser escritas da seguinte forma:

$$
\begin{aligned}
& \rho\left(\frac{\partial u}{\partial t}+u . \nabla u\right)=-\nabla p+\mu \Delta u+F \\
& \nabla . u=q(x, t)
\end{aligned}
$$


Na equação (2.19), $q(x, t)$ é uma função escalar que modela a ação das fontes e dos sorvedouros. Adota-se para essa função a mesma forma empregada por Peskin [25] na simulação do fluxo sangüíneo no lado esquerdo do coração. Assim:

$$
q(x, t)=\sum_{m=1}^{k} Q_{m}(t) \Psi_{m}(x)
$$

onde $Q_{m}(t)$ é a vazão do escoamento associada à m-ésima fonte e ao m-ésimo sorvedouro no instante t e $\Psi_{m}(x)$ é uma função peso que especifica a distribuição espacial da m-ésima fonte e do m-ésimo sorvedouro.

A função peso $\Psi_{m}(x)$ é dada por

$$
\Psi_{m}(x)=W_{F_{m}}(x)-W_{S_{m}}(x)
$$

sendo $W_{F_{m}}$ e $W_{S_{m}}$ funções não negativas, com suportes compactos e integrais iguais a um. Os suportes compactos $V_{P_{F_{m}}}$ da m-ésima fonte e $V_{P_{S_{m}}}$ do m-ésimo sorvedouro são quadrados de lado $4 h_{o}$, centrados respectivamente em $P_{F_{m}}$ e $P_{S_{m}}$, centros da m-ésima fonte e do m-ésimo sorvedouro. Dessa forma, pode-se reescrever a expressão (2.20) para a função $\Psi_{m}(x)$ como:

$$
\Psi_{m}(x)= \begin{cases}W_{F_{m}}\left(x-P_{F_{m}}\right), & \forall x \in V_{P_{F_{m}}} \\ W_{S_{m}}\left(x-P_{S_{m}}\right), & \forall x \in V_{P_{S_{m}}} \\ 0, & \text { caso contrário }\end{cases}
$$

Para as funções $W_{F_{m}}(x)$ e $W_{S_{m}}(x)$ emprega-se

$$
W_{F_{m}}(x)=W_{S_{m}}(x)=\delta_{h_{o}}(x)
$$

onde $\delta_{h_{o}}(x)$ é uma aproximação do delta de Dirac e $h_{o}$ é a medida empregada no lado do quadrado suporte das funções $W_{F_{m}}(x)$ e $W_{S_{m}}(x)$.

A Figura 2.7 ilustra a ação da m-ésima fonte e do m-ésimo sorvedouro no escoamento bem como identifica os suportes das funções $W_{F_{m}}(x)$ e $W_{S_{m}}(x)$.

Para a vazão $Q_{m}(t)$ adota-se o modelo de resistência linear proveniente da lei de Hagen-Poiseuille - equação (A.1):

$$
Q=\frac{p_{1}-p_{2}}{R}=\frac{\Delta p}{R}
$$




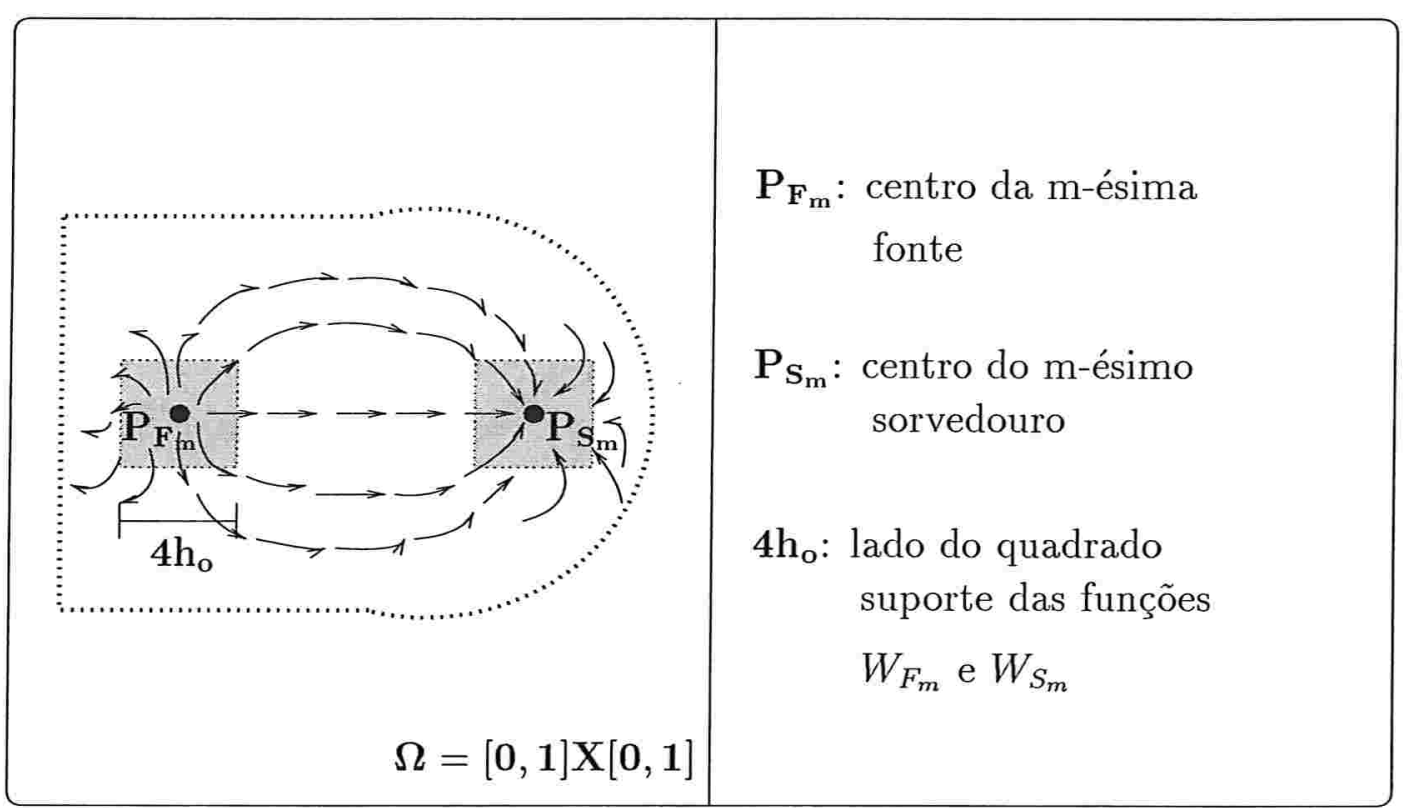

Figura 2.7: Ação da m-ésima fonte e do m-ésimo sorvedouro

$\operatorname{com} \Delta p=p_{1}-p_{2}$ e $R=\frac{8 \mu L}{\pi r^{4}}$.

Assim:

$$
Q_{m}(t)=\frac{\bar{P}_{m}-P_{m}(t)}{R_{m}}
$$

onde $\bar{P}_{m}$ e $R_{m}$ são constantes relacionadas ao problema que se pretende modelar.

Em relação à pressão $P_{m}(t)$ presente na equação (2.22), sabe-se que esta é dada pela diferença entre a pressão média na m-ésima fonte e no m-ésimo sorvedouro. Portanto:

$$
P_{m}(t)=P_{F_{m}}(t)-P_{S_{m}}(t)
$$

Como $W_{F}$ e $W_{S}$ são funções peso, então as pressões na m-ésima fonte e no m-ésimo sorvedouro são naturalmente definidas como:

$$
\begin{aligned}
& P_{F_{m}}(t)=\int_{\Omega} p(x, t) W_{F_{m}}(x) d x \\
& P_{S_{m}}(t)=\int_{\Omega} p(x, t) W_{S_{m}}(x) d x
\end{aligned}
$$

com $p(x, t)$ representando a pressão no fluido.

Logo, com o emprego de (2.24) e (2.25), a expressão (2.23) pode ser reescrita da 
seguinte forma:

$$
\begin{aligned}
& P_{m}(t)=P_{F_{m}}(x)-P_{S_{m}}(x) \\
& P_{m}(t)=\int_{\Omega} p(x, t) W_{F_{m}}(x) d x-\int_{\Omega} p(x, t) W_{S_{m}}(x) d x \\
& \quad P_{m}(t)=\int_{\Omega} p(x, t)\left[W_{F_{m}}(x)-W_{S_{m}}(x)\right] d x
\end{aligned}
$$

Substituindo a expressão (2.20) em (2.26) chega-se a:

$$
P_{m}(t)=\int_{\Omega} p(x, t) \Psi_{m}(x) d x
$$

Da expressão (2.22) tem-se que:

$$
\begin{gathered}
Q_{m}(t)=\frac{\bar{P}_{m}-P_{m}(t)}{R_{m}} \\
P_{m}(t)=\bar{P}_{m}-R_{m} Q_{m}(t)
\end{gathered}
$$

Comparando-se as expressões (2.27) e (2.28) conclui-se que:

$$
P_{m}(t)=\int_{\Omega} p(x, t) \Psi_{m}(x) d x=\bar{P}_{m}-R_{m} Q_{m}(t)
$$

Dessa maneira, conhecidas a pressão $p$ no fluido, as constantes $\bar{P}_{m}$ e $R_{m}$ e a função $\Psi_{m}$ associadas a cada fonte e sorvedouro, pode-se calcular a vazão $Q_{m}$ e, conseqüentemente, a função $q(x, t)$ que modela a ação das fontes e dos sorvedouros.

Resta ainda discutir o primeiro passo na aplicação do método da fronteira imersa, o qual consiste no cálculo da força exercida pelos pontos que representam a fronteira elástica - equaçao (2.16).

\subsubsection{Força elástica}

A fronteira pode ser vista como um conjunto de molas conectadas, que se expande com a velocidade das partículas do fluido na qual se encontra imersa dependendo dos valores empregados para a elasticidade. 
Para conferir rigidez à fronteira, utiliza-se classicamente um tipo de força restauradora para a densidade de força elástica $f$ dada por

$$
f(s, t)=-\mathrm{k}\left(X(s, t)-X_{o}(s)\right)
$$

Na expressão (2.30), $X(s, t)$ é a posição da fronteira em cada instante de tempo, $X_{o}(s)$ é a posição de equilíbrio que se deseja que a fronteira mantenha e k é uma constante de proporcionalidade. Esse tipo de força obedece a lei de Hooke, segundo a qual o termo "força" deve ser interpretado de uma maneira ampla, como torque, pressão ou qualquer fator que provoque distorção. Se a força se limita a "puxões" ou empurrões", onde a deformação é simplesmente o deslocamento do ponto de aplicação da força, esta e o deslocamento são relacionados por

$$
F=\mathrm{k} x
$$

onde k é uma constante de proporcionalidade chamada constante de força e $x$ é o deslocamento a partir da posição de equilíbrio - Sears [35]. Na equação anterior, $F$ representa a força que deve ser exercida sobre o corpo elástico para causar o deslocamento $x$. A força com a qual o corpo elástico puxa um objeto a ele preso é chamada força restauradora. Esta força equivale a

$$
F_{\text {rest }}=-\mathrm{k} x
$$

Assim, o emprego de uma força como a descrita pela equação (2.30) faz com que um ponto que se encontre fora da posição de equilíbrio retorne para a posição anterior.

Ao utilizar a expressão (2.30) com $\mathrm{k}=10^{6} \frac{d y n}{\mathrm{~cm}}$, Remigio [29] modelou a rigidez da fronteira imersa, ou seja, os pontos que representam a presença da fronteira mantiveram sua posição inicial no decorrer do tempo, não sendo afetados pela velocidade das partículas do fluido, ou seja, não sendo arrastados pelo fluxo.

Para o problema que se pretende simular, a rigidez é desejada apenas para uma parte da fronteira - segmentos $\overline{A B}, \overline{A C}$ e $\overline{C D}$, justamente aquela que representa o bronquíolo respiratório. Em relação à porção da fronteira que representa a superfície alveolar segmento $\overline{B D}$, espera-se uma certa mobilidade dos pontos que a representam.

$\mathrm{Na}$ modelagem da expansão alveolar emprega-se uma força do tipo restauradora para conferir rigidez à porção da fronteira imersa que representa o bronquíolo respiratório, e 
diferentes funções para alterar a elasticidade dos pontos do segmento $\overline{B D}$ ao longo do tempo, o que possibilita simular a expansão de um alvéolo ou de um saco alveolar. 


\section{Discretização das equações do modelo}

As equações apresentadas no segundo capítulo precisam ser discretizadas para compor o método computacional a ser empregado na simulação da expansão da fibra elástica. A solução das equações (2.13) a (2.17) é aproximada pelo emprego de diferenças finitas em uma malha computacional Euleriana-Lagrangeana de tamanho $N \times N$.

No domínio periódico $\Omega=[0,1] X[0,1]$ considera-se uma malha uniforme de passo espacial $h=\Delta x=\Delta y=\frac{1}{N}$, esquematizada na Figura 3.1. Cada ponto $x_{i, j}$ da malha Euleriana é dado por

$$
x_{i, j}=\left(x_{i}, y_{j}\right)=(i . h, j . h)
$$

$\operatorname{com} i, j=0,1, \ldots, N-1$.

A configuração inicial da fronteira, de comprimento total $S=[a, b]$, é discretizada em um conjunto de $N_{B}$ pontos. Contudo, a fronteira possui apenas $N_{B}-1$ pontos, uma vez que suas extremidades coincidem pelo fato dela ser fechada. Então

$$
X\left(s_{o}, t\right)=X\left(s_{N_{B}}, t\right), \forall t \geq 0
$$

sendo $s \in S$ o parâmetro Lagrangeano que permite verificar a posição $X_{l}$ dos pontos da fronteira no decorrer do tempo dado por

$$
s_{l}=a+l \cdot \Delta s
$$

onde $\Delta s$ é a distância entre os pontos da fronteira imersa, $l=0,1, \ldots, N_{B}-1, s_{o}=a$ e $s_{N_{B}}=b$. 


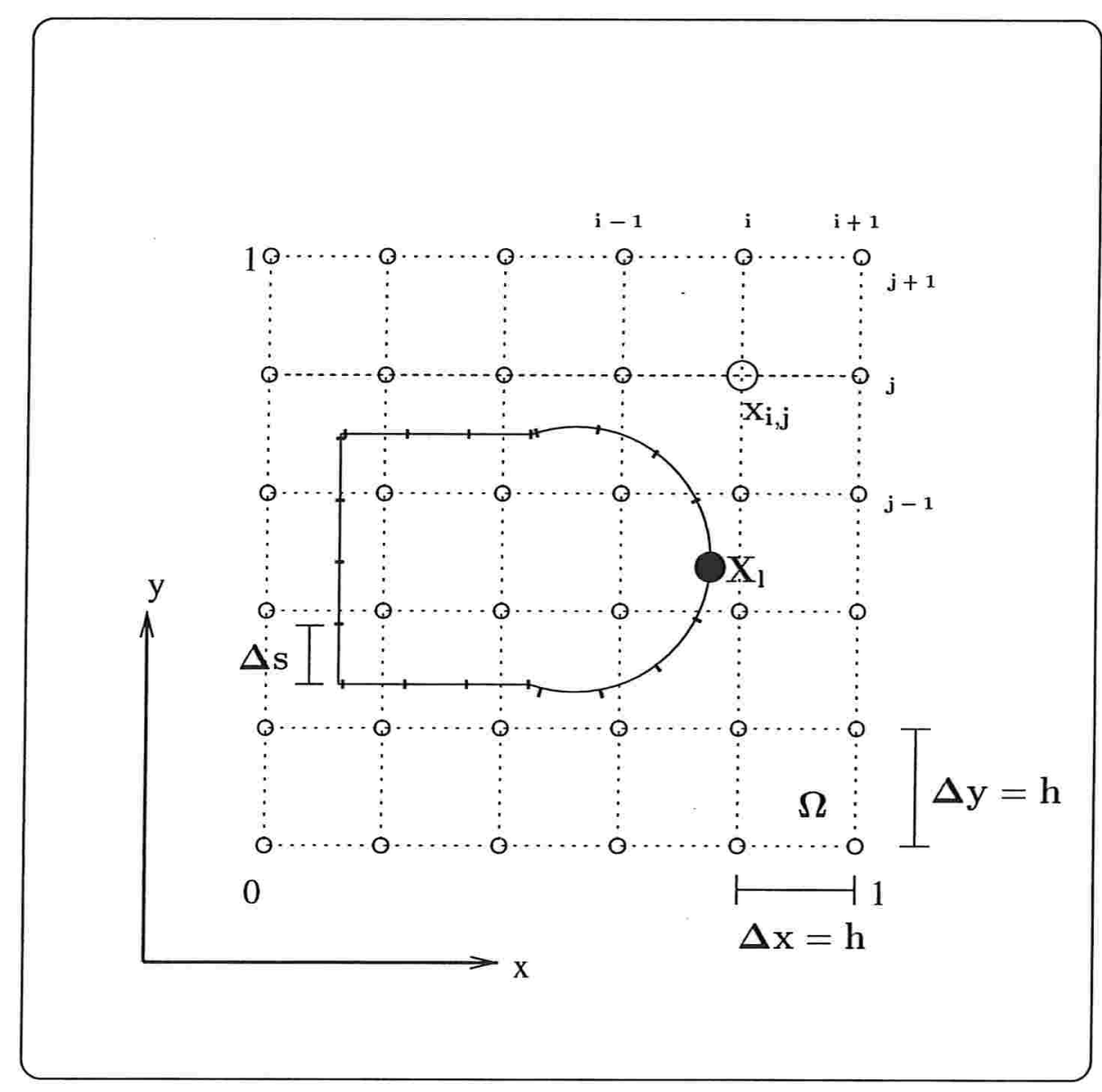

Figura 3.1: Malha computacional para $\mathrm{n}=5 \mathrm{e} \mathrm{h}=0.2$

A distância $\Delta s$ é dada por

$$
\Delta s=\frac{b-a}{N_{B}-1}
$$

e no método da fronteira imersa toma-se um número de pontos $N_{B}$ para a fronteira tal que

$$
\Delta s \leq \frac{h}{2}
$$

Dessa maneira, empregou-se nas silumações efetuadas $\Delta s=\frac{h}{2}=\frac{1}{2 N}$, sendo que este $\Delta s$ representa a distância entre dois pontos consecutivos na configuração inicial da fronteira (retângulo $A B C D$ ). Como a posição dos pontos do segmento $\overline{B D}$ varia no decorrer do tempo, a medida $\Delta s$ não corresponde à distância entre os pontos dessa parte da fronteira a cada instante de tempo. Poder-se-ia então prever o comprimento $S=[a, b]$ da fronteira imersa no instante final $t=1.5 \mathrm{~s}$, o que permitiria inserir um número de pontos suficiente para garantir a condição $\Delta s \leq \frac{h}{2}$ ao longo de toda fronteira a cada passo no tempo. 
A velocidade e a pressão, periódicas na fronteira do domínio, ou seja, nos pontos

$$
\begin{aligned}
& \left(x_{o}, y_{j}\right) \text { e }\left(x_{N}, y_{j}\right) \\
& \left(x_{i}, y_{o}\right) \text { e }\left(x_{i}, y_{N}\right)
\end{aligned}
$$

são calculadas nos tempos

$$
t_{n}=n . \Delta t
$$

onde $\Delta t$ é o passo no tempo e $t_{n}$ é o tempo computado em n passos.

O passo temporal $\Delta t$ é dado por

$$
\Delta t=\min \left\{\frac{h^{2}}{2}, \sqrt{\frac{8 . h}{3 . K_{\text {elast }}}}\right\}
$$

onde

$$
\Delta t=\frac{h^{2}}{2}
$$

garante estabilidade uma vez que $\Delta t \leq(\Delta x)^{2}=h^{2}$ é a condição CFL $^{1}$ para o esquema explícito e

$$
\Delta t \leq \sqrt{\frac{8 \cdot \Delta x}{3 \cdot \mathrm{K}_{\text {elast }}}}=\sqrt{\frac{8 \cdot h}{3 \cdot \mathrm{K}_{\text {elast }}}}
$$

é a condição de estabilidade determinada por Ming-Chih [21] para o esquema explícito do método da fronteira imersa em uma dimensão. Neste trabalho essa condição também foi adotada para duas dimensões e $\mathrm{K}_{\text {elast }}=\max \left\{\mathrm{k}_{1}, \mathrm{k}_{2}, \ldots, \mathrm{k}_{n}\right\}$, uma vez que são atribuídos $\mathrm{n}$ valores distintos para a elasticidade na modelagem da rigidez e da flexibilidade da fronteira imersa.

No método da fronteira imersa, a configuração inicial da fronteira é empregada para calcular o campo de pressões. Este permite o cálculo do campo de velocidades, o qual por sua vez possibilita a atualização dos pontos da fronteira imersa. Assim, o método computacional deve calcular o campo de pressões, o campo de velocidades e a posição da fronteira imersa. Estas variáveis, bem como outras grandezes envolvidas nas equações (2.13) a (2.17) são aproximadas computacionalmente empregando-se as notações presentes na Tabela 3.1.

\footnotetext{
${ }^{1}$ Courant, Friedrichs e Lewy
} 


\begin{tabular}{|l|l|l|}
\hline Variáveis/Grandezas & Notação & Variação \\
\hline Velocidade $u_{i, j}^{n}$ & $u\left(x_{i}, y_{j}, t_{n}\right)$ & $i, j=0,1, \ldots, N-1$ \\
\hline Pressão $p_{i, j}^{n}$ & $p\left(x_{i}, y_{j}, t_{n}\right)$ & $i, j=0,1, \ldots, N-1$ \\
\hline Força exercida pela fibra $F_{i, j}^{n}$ & $F\left(x_{i}, y_{j}, t_{n}\right)$ & $i, j=0,1, \ldots, N-1$ \\
\hline Posição de um ponto da fibra $X_{l}$ & $X\left(s_{l}, t_{n}\right)$ & $l=0,1, \ldots, N_{B}-1$ e $n=0,1, \ldots$ \\
\hline Densidade de força elástica $f_{l}^{n}$ & $f\left(s_{l}, t_{n}\right)$ & $l=0,1, \ldots, N_{B}-1$ e $n=0,1, \ldots$ \\
\hline
\end{tabular}

Tabela 3.1: Notação empregada

Os operadores de diferenças finitas são discretizados da forma clássica:

Primeira derivada centrada

$$
\begin{aligned}
D_{x}^{o} \phi_{i, j} & =\frac{\phi_{i+1, j}-\phi_{i-1, j}}{2 h} \\
D_{y}^{o} \phi_{i, j} & =\frac{\phi_{i, j+1}-\phi_{i, j-1}}{2 h}
\end{aligned}
$$

Primeira derivada progressiva

$$
\begin{aligned}
D_{x}^{+} \phi_{i, j} & =\frac{\phi_{i+1, j}-\phi_{i, j}}{h} \\
D_{y}^{+} \phi_{i, j} & =\frac{\phi_{i, j+1}-\phi_{i, j}}{h} \\
D_{s}^{+} \Psi_{l} & =\frac{\Psi_{l+1}-\Psi_{l}}{\Delta s}
\end{aligned}
$$

Primeira derivada regressiva

$$
\begin{aligned}
D_{x}^{-} \phi_{i, j} & =\frac{\phi_{i, j}-\phi_{i-1, j}}{h} \\
D_{y}^{-} \phi_{i, j} & =\frac{\phi_{i, j}-\phi_{i, j-1}}{h} \\
D_{s}^{-} \Psi_{l} & =\frac{\Psi_{l}-\Psi_{l-1}}{\Delta s}
\end{aligned}
$$

Segunda derivada

$$
\begin{aligned}
& D_{x}^{+} D_{x}^{-} \phi_{i, j}=D_{x}^{+}\left[\frac{\phi_{i, j}-\phi_{i-1, j}}{h}\right]=\frac{\phi_{i+1, j}+\phi_{i-1, j}-2 \phi_{i, j}}{h^{2}} \\
& D_{y}^{+} D_{y}^{-} \phi_{i, j}=D_{y}^{+}\left[\frac{\phi_{i, j}-\phi_{i, j-1}}{h}\right]=\frac{\phi_{i, j+1}+\phi_{i, j-1}-2 \phi_{i, j}}{h^{2}}
\end{aligned}
$$




\section{Gradiente}

$$
(G \phi)_{i, j}=\left(D_{x}^{o}, D_{y}^{o}\right) \phi_{i, j}
$$

Divergente

$$
D \cdot u_{i, j}=D_{x}^{o} u_{i, j}+D_{y}^{o} v_{i, j}
$$

\section{Laplaciano}

$$
\Delta_{h} \phi_{i, j}=\left(D_{x}^{+} D_{x}^{-}+D_{y}^{+} D_{y}^{-}\right) \phi_{i, j}
$$

As aproximações dadas por (3.4) a (3.9) são de primeira ordem, enquanto que aquelas fornecidas por (3.2) e (3.3) são de segunda ordem. As aproximações para os operadores diferenciais gradiente (3.12), divergente (3.13) e Laplaciano (3.14) são de segunda ordem.

A plicando-se o operador divergente (3.13) ao operador gradiente (3.12) e utilizando as discretizações dadas por (3.2) e (3.3), obtém-se:

$$
\begin{aligned}
D . G \phi_{i, j}= & D \cdot\left(D_{x}^{o}, D_{y}^{o}\right) \phi_{i, j} \\
D . G \phi_{i, j}= & D_{x}^{o}\left(D_{x}^{o} \phi_{i, j}\right)+D_{y}^{o}\left(D_{y}^{o} \phi_{i, j}\right) \\
D . G \phi_{i, j}= & D_{x}^{o}\left(\frac{\phi_{i+1, j}-\phi_{i-1, j}}{2 h}\right)+D_{y}^{o}\left(\frac{\phi_{i, j+1}-\phi_{i, j-1}}{2 h}\right) \\
D . G \phi_{i, j}= & \frac{1}{2 h}\left(\frac{\phi_{i+1+1, j}-\phi_{i-1+1, j}}{2 h}-\frac{\phi_{i+1-1, j}-\phi_{i-1-1, j}}{2 h}\right)+ \\
& +\frac{1}{2 h}\left(\frac{\phi_{i, j+1+1}-\phi_{i, j-1+1}}{2 h}-\frac{\phi_{i, j+1-1}-\phi_{i, j-1-1}}{2 h}\right) \\
D . G \phi_{i, j}= & \frac{1}{2 h}\left(\frac{\phi_{i+2, j}-\phi_{i, j}-\phi_{i, j}+\phi_{i-2, j}+\phi_{i, j+2}-\phi_{i, j}-\phi_{i, j}+\phi_{i, j-2}}{2 h}\right) \\
& D . G \phi_{i, j}=\frac{\phi_{i+2, j}+\phi_{i-2, j}+\phi_{i, j+2}+\phi_{i, j-2}-4 \phi_{i, j}}{4 h^{2}}
\end{aligned}
$$

A expressão (3.15) fornece um operador de diferenças finitas para o Laplaciano semelhante à regra dos cinco pontos.

As equações de interação fluido-fibra (2.15) e (2.17) exigem, respectivamente, a conversão da formulação Lagrangeana para a Euleriana e vice-versa. Para isso, emprega-se o delta de Dirac $\delta$ bidimensional, o qual é aproximado por um delta discreto $\delta_{h_{\circ}}$ equivalente ao produto de duas funções discretas unidimensionais, ou seja

$$
\delta_{h_{o}}\left(x_{i}, y_{j}\right)=d_{h_{o}}\left(x_{i}\right) \cdot d_{h_{o}}\left(y_{j}\right)
$$


Adota-se aqui

$$
d_{h_{o}}(x)= \begin{cases}\frac{1}{4 h_{o}}\left(1+\cos \left(\frac{\pi x}{2 h_{\circ}}\right)\right), & \text { se }|x|<2 h_{o} \\ 0, & \text { se }|x| \geq 2 h_{o}\end{cases}
$$

devido ao conjunto de propriedades de compatibilidade discretas descritas por Peskin $[23]^{2}$.

A Figura 3.2 ilustra o delta de Dirac unidimensional (3.17) e bidimensional (3.16) para $n=64, h=\frac{1}{64}$ e suporte $h_{o}=\frac{1}{32}$.

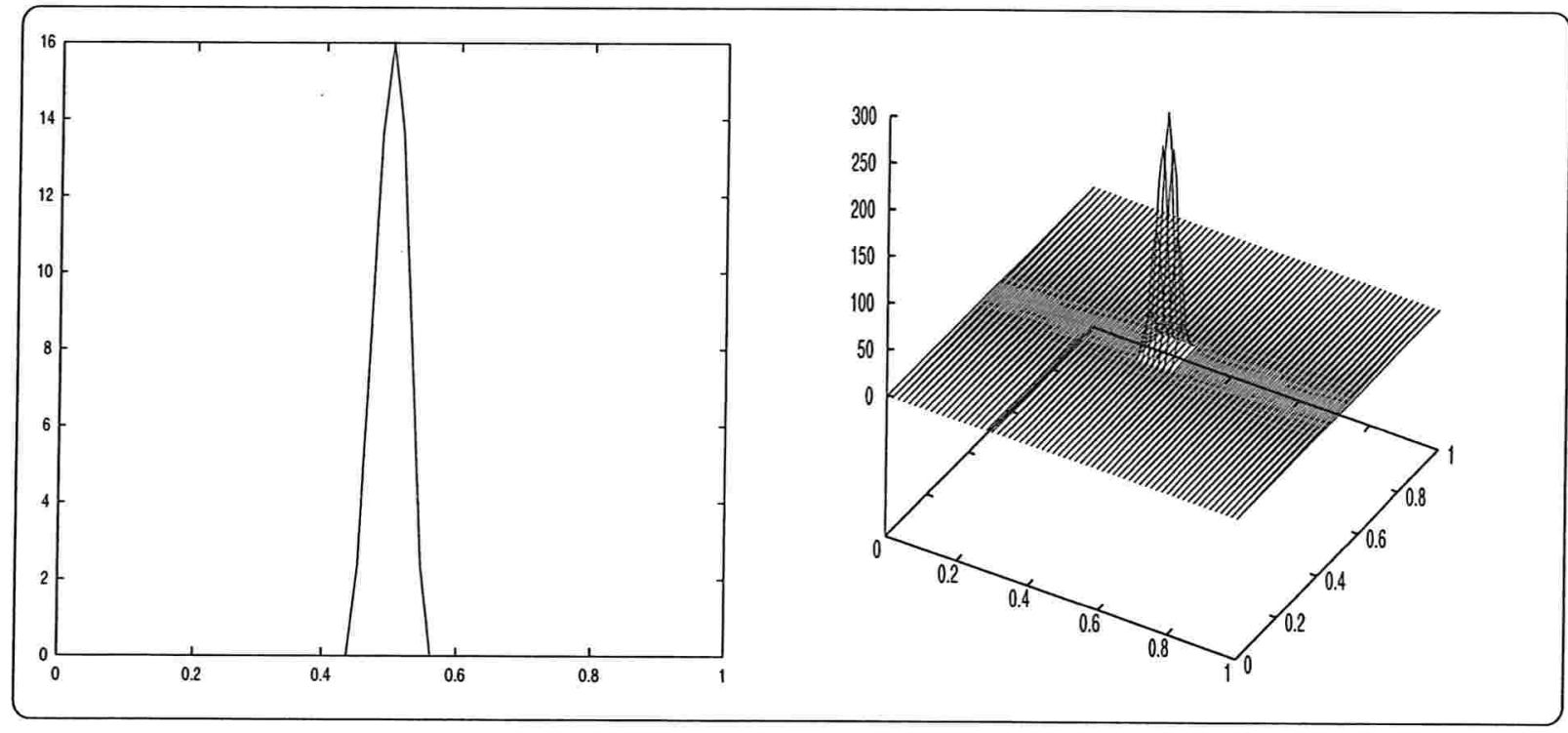

Figura 3.2: Delta de Dirac unidimensional com centro em $x=0.5$ e bidimensional com centro em $(x=0.5, y=0.5)$

O delta discreto (3.16) permite interpolar forças e velocidades entre pontos da fibra e do fluido em uma vizinhança de tamanho $4 h_{o} \mathrm{x} 4 h_{o}$, uma vez que a função discreta unidimensional (3.17) tem suporte $\left[-2 h_{o}, 2 h_{o}\right]$. Assim, a força $f_{l}$ exercida por um ponto $X_{l}$ qualquer da fibra é difundida aos pontos $x_{i, j}$ do fluido que estão dentro do quadrado de lado $4 h_{o}$ e cujo centro é o ponto da fibra. Ao mesmo tempo, as velocidades $u_{i, j}$ desses pontos do fluido são utilizadas para interpolar a velocidade no ponto da fibra. A Figura 3.3 detalha as interpolações efetuadas com o uso do delta de Dirac.

$$
{ }^{2} h_{o}=h=\frac{1}{N}
$$




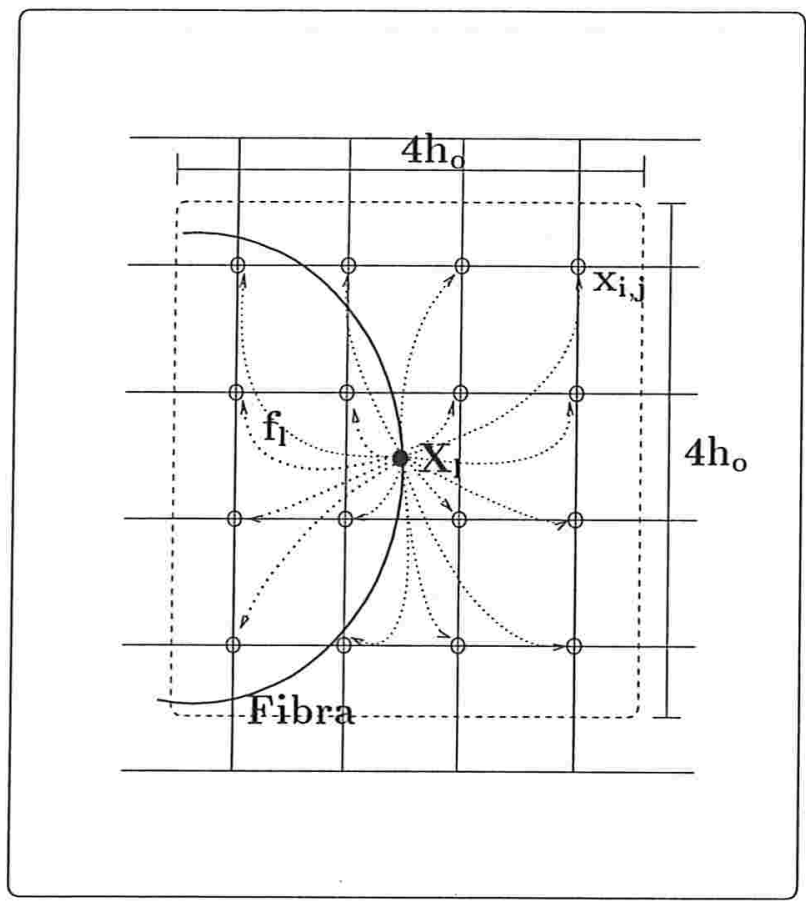

A

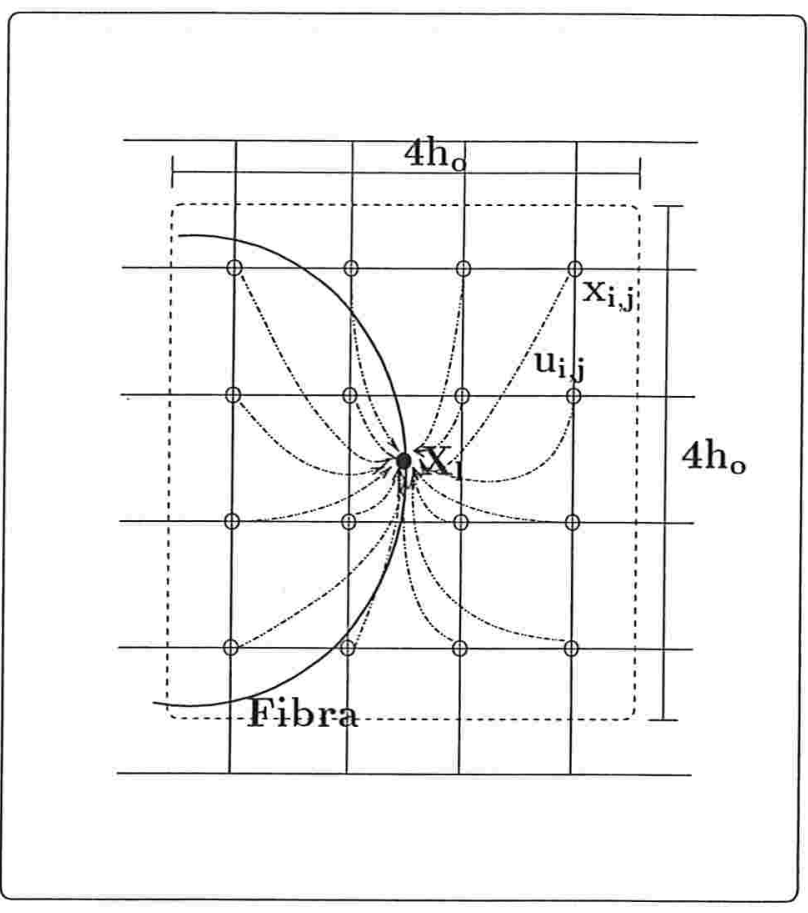

$\mathrm{B}$

Figura 3.3: Interpolações efetuadas com o emprego do delta de Dirac: (A) distribuição da densidade de força elástica $f_{l}$ exercida pelo ponto $X_{l}$ da fibra para os pontos $x_{i, j}$ do fluido pertencentes à região interna ao quadrado de lado $4 h_{o}$ do qual $X_{l}$ é o centro; (B) cálculo da velocidade do ponto $X_{l}$ da fibra a partir da velocidade $u_{i, j}$ dos pontos $x_{i, j}$ do fluido pertencentes à região interna ao quadrado de lado $4 h_{o}$ do qual $X_{l}$ é o centro

\subsection{Equações do fluido}

As equações do fluido (2.13) e (2.14) são resolvidas empregando-se um método de projeção que é uma variação do método de projeção de Chorin. O método de Chorin [4] [27] é um método explícito de passo fracionário, de primeira ordem no tempo e segunda ordem no espaço, cuja característica principal consiste na supressão do gradiente de pressão no primeiro passo e a inclusão do mesmo para correção no segundo passo. Em cada instante de tempo, o campo deve ser livre de divergência.

As equações do fluido são então solucionadas em duas etapas.

Na primeira etapa, calcula-se o campo de velocidades auxiliar $u^{n+1,2}$ empregando o 
algoritmo de Printz [26]:

$$
\begin{aligned}
& \rho\left(\frac{u^{n+1,0}-u^{n}}{\Delta t}\right)=F^{n} \\
& \rho\left(\frac{u^{n+1,1}-u^{n+1,0}}{\Delta t}+u^{n} D_{x}^{o} u^{n+1,1}\right)=\mu D_{x}^{+} D_{x}^{-} u^{n+1,1} \\
& \rho\left(\frac{u^{n+1,2}-u^{n+1,1}}{\Delta t}+v^{n} D_{y}^{o} u^{n+1,2}\right)=\mu D_{y}^{+} D_{y}^{-} u^{n+1,2}
\end{aligned}
$$

$\operatorname{com} \mathrm{u}^{m}=\left(u^{m}, v^{m}\right)$.

Na segunta etapa, o campo de velocidades auxiliar $u^{n+1,2}$ é decomposto em um campo de velocidades $u^{n+1}$ com divergente nulo e no gradiente do campo escalar $p^{n+1}$ :

$$
\begin{aligned}
& G p^{n+1}=\frac{\rho}{\Delta t}\left(u^{n+1,2}-u^{n+1}\right) \\
& D \cdot u^{n+1}=0
\end{aligned}
$$

Desenvolvendo os membros da equação (3.19) e empregando-se as discretizações dadas por (3.2), (3.4) e (3.7), chega-se a:

$$
\begin{aligned}
& \rho\left[\frac{u_{i, j}^{n+1,1}-u_{i, j}^{n+1,0}}{\Delta t}+u^{n}\left(\frac{u_{i+1, j}^{n+1,1}-u_{i-1, j}^{n+1,1}}{2 h}\right)\right]=\mu D_{x}^{+}\left(\frac{u_{i, j}^{n+1,1}-u_{i-1, j}^{n+1,1}}{h}\right) \\
& \frac{\rho}{\Delta t} u_{i, j}^{n+1,1}-\frac{\rho}{\Delta t} u_{i, j}^{n+1,0}+\frac{\rho u^{n}}{2 h} u_{i+1, j}^{n+1,1}-\frac{\rho u^{n}}{2 h} u_{i-1, j}^{n+1,1}=\mu\left(\frac{u_{i+1, j}^{n+1,1}-u_{i-1+1, j}^{n+1,1}}{h^{2}}-\frac{u_{i, j}^{n+1,1}-u_{i-1, j}^{n+1,1}}{h^{2}}\right) \\
& \frac{\rho}{\Delta t} u_{i, j}^{n+1,1}-\frac{\rho}{\Delta t} u_{i, j}^{n+1,0}+\frac{\rho u^{n}}{2 h} u_{i+1, j}^{n+1,1}-\frac{\rho u^{n}}{2 h} u_{i-1, j}^{n+1,1}=\frac{\mu}{h^{2}} u_{i+1, j}^{n+1,1}-\frac{\mu}{h^{2}} u_{i, j}^{n+1,1}-\frac{\mu}{h^{2}} u_{i, j}^{n+1,1}+\frac{\mu}{h^{2}} u_{i-1, j}^{n+1,1} \\
& -\left(\frac{\mu}{h^{2}}+\frac{\rho u^{n}}{2 h}\right) u_{i-1, j}^{n+1,1}+\left(\frac{\rho}{\Delta t}+\frac{2 \mu}{h^{2}}\right) u_{i, j}^{n+1,1}-\left(\frac{\mu}{h^{2}}-\frac{\rho u^{n}}{2 h}\right) u_{i+1, j}^{n+1,1}=\frac{\rho}{\Delta t} u_{i, j}^{n+1,0}
\end{aligned}
$$

Multiplicando-se ambos os lados da igualdade acima por $\frac{\Delta t}{\rho}$ :

$$
-\left(\frac{\Delta t \mu}{\rho h^{2}}+\frac{\Delta t u^{n}}{2 h}\right) u_{i-1, j}^{n+1,1}+\left(1+\frac{2 \Delta t \mu}{\rho h^{2}}\right) u_{i, j}^{n+1,1}-\left(\frac{\Delta t \mu}{\rho h^{2}}-\frac{\Delta t u^{n}}{2 h}\right) u_{i+1, j}^{n+1,1}=u_{i, j}^{n+1,0}
$$


Considerando-se as igualdades:

$$
\begin{aligned}
A[i] & =\frac{\Delta t \mu}{\rho h^{2}}+\frac{\Delta t u^{n}}{2 h} \\
B[i] & =1+\frac{2 \Delta t \mu}{\rho h^{2}} \\
C[i] & =\frac{\Delta t \mu}{\rho h^{2}}-\frac{\Delta t u^{n}}{2 h} \\
D[i] & =u_{i, j}^{n+1,0}
\end{aligned}
$$

a expressão (3.23) pode ser reescrita como:

$$
-A[i] u_{i-1, j}^{n+1,1}+B[i] u_{i, j}^{n+1,1}-C[i] u_{i+1, j}^{n+1,1}=D[i]
$$

Substituindo-se de forma análoga as discretizações fornecidas por (3.3), (3.5) e (3.8) na equação (3.20), obtém-se:

$$
-A^{\prime}[i] u_{i, j-1}^{n+1,2}+B^{\prime}[i] u_{i, j}^{n+1,2}-C^{\prime}[i] u_{i, j+1}^{n+1,2}=D^{\prime}[i]
$$

As expressões (3.24) e (3.25) caracterizam sistemas tridiagonais periódicos, os quais serão solucionados empregando-se o algoritmo de Thomas (Apêndice C).

Aplicando-se o operador divergente (3.13) à expressão (3.21) e usando (3.22), chega-se $\grave{a}$

$$
\begin{gathered}
D . G p^{n+1}=\frac{\rho}{\Delta t}\left(D \cdot u^{n+1,2}-D \cdot u^{n+1}\right) \\
\text { D.G } p^{n+1}=\frac{\rho}{\Delta t} D \cdot u^{n+1,2}
\end{gathered}
$$

a qual constitui uma equação de Poisson para a pressão $\left(\frac{\partial^{2} p}{\partial x^{2}}(x, t)+\frac{\partial^{2} p}{\partial y^{2}}(x, t)=F(x, y, t) \neq\right.$ 0 ). Esta será solucionada através do emprego da FFT ou transformada rápida de Fourier (http://theory.lcs.mit.edu/fftw) devido ao fato do domínio ser periódico e o passo espacial $h$ ser o mesmo nas direções $x$ e $y$.

Assim, as duas etapas de resolução das equações de Navier-Stokes permitem atualizar o campo de pressões e o campo de velocidades. 


\subsection{Equações de interação fluido-fibra}

A equação (2.15)

$$
F(x, t)=\int_{S} f(s, t) \delta(x-X(s, t)) d s
$$

é aproximada por

$$
F_{i, j}^{n}=\sum_{l} f_{l}^{n} \delta_{h_{o}}\left(x_{i, j}-X_{l}^{n}\right) \Delta s
$$

Já a equação (2.17)

$$
\frac{\partial X}{\partial t}(s, t)=\int_{\Omega} u(x, t) \delta(x-X(s, t)) d x
$$

é discretizada da seguinte maneira:

$$
\begin{gathered}
D_{t}^{+} X_{l}^{n+1}=\sum_{i, j} u_{i, j}^{n+1} \delta_{h_{o}}\left(x_{i, j}-X_{l}^{n}\right) h^{2} \\
\frac{X_{l}^{n+1}-X_{l}^{n}}{\Delta t}=\sum_{i, j} u_{i, j}^{n+1} \delta_{h_{o}}\left(x_{i, j}-X_{l}^{n}\right) h^{2} \\
X_{l}^{n+1}=X_{l}^{n}+\Delta t \sum_{i, j} u_{i, j}^{n+1} \delta_{h_{o}}\left(x_{i, j}-X_{l}^{n}\right) h^{2}
\end{gathered}
$$

O delta de Dirac não precisa ser discretizada da mesma forma nas equações (3.27) e (3.28). Contudo, quando isso ocorre a parcela de potência proveniente da ação da força elástica é integralmente transferida ao fluido.

Até aqui, as equações do método da fronteira imersa foram discretizadas sem considerar as alterações provenientes da inserção de fontes e sorvedouros no domínio computacional. Os passos a serem seguidos na execução do método estão indicados no algoritmo que segue.

\subsection{Algoritmo}

O algoritmo estruturado a seguir ilustra visualmente os passos a serem adotados na aplicação do método da fronteira imersa. 


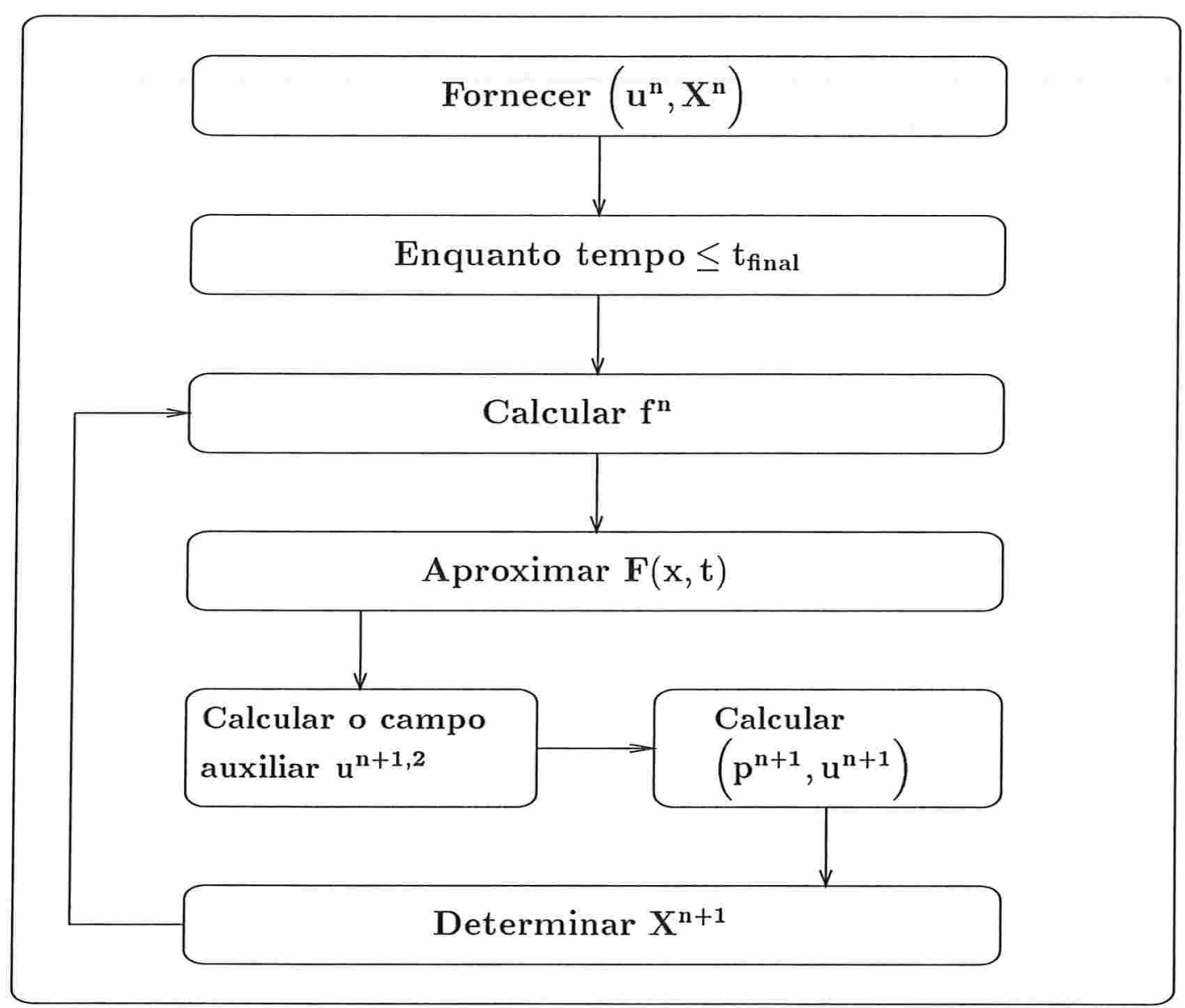

Figura 3.4: Algoritmo estruturado do método da fronteira imersa

A cada passo no tempo tem-se que atualizar o campo de velocidades $u$ e a posição $X$ dos pontos da fibra imersa. Logo, dado

$$
\left(u^{n}, X^{n}\right)
$$

deve-se calcular

$$
\left(u^{n+1}, X^{n+1}\right)
$$

Isto é feito em quatro passos.

1으 passo: Calcular a densidade de força elástica $f^{n}$. A modelagem dessa força depende do problema que se pretende simular. 
$2^{\text {o }}$ passo: Distribuir a densidade de força elástica exercida pela fronteira imersa aos pontos do fluido por intermédio da equação (3.27).

3o- passo: Solucionar as equações de Navier-Stokes empregando uma variação do método de projeção de Chorin.

1a etapa: Calcular o campo de velocidades auxiliar $u^{n+1,2}$ através das equações (3.18), (3.19) e (3.20). As equações (3.19) e (3.20) geram sistemas tridiagonais periódicos, os quais são solucionados através do algoritmo de Thomas.

$2^{\text {a }}$ etapa: Decompor o campo de velocidades auxiliar $u^{n+1,2}$ em um campo de velocidades $u^{n+1}$ com divergente nulo e no gradiente do campo escalar $p^{n+1}$ utilizando as equações (3.21) e (3.22). Estas equações originam uma Poisson - equação (3.26) - para a pressão, a qual é solucionada com o uso da transformada rápida de Fourier (FFT).

4º passo: Atualizar a posição dos pontos da fronteira imersa usando a equação (3.28).

\subsection{Introdução de fontes e sorvedouros}

A introdução de fontes e sorvedouros no modelo modifica o $3^{\circ}$ passo do algoritmo descrito anteriormente, ou seja, a resolução das equações de Navier-Stokes.

Na segunda etapa desse passo, as equações (3.21) e (3.22) passam a ser escritas como:

$$
\begin{aligned}
& G p^{n+1}=\frac{\rho}{\Delta t}\left(u^{n+1,2}-u^{n+1}\right) \\
& D . u^{n+1}=\sum_{m=1}^{k} Q_{m}^{n+1} \Psi_{m}
\end{aligned}
$$

onde $u^{n+1,2}$ é o campo de velocidades auxiliar calculado na primeira etapa do passo e $q(x, t)=\sum_{m=1}^{k} Q_{m}^{n+1} \Psi_{m}$ é a função que modela a ação das fontes e dos sorvedouros.

As equações (3.29) e (3.30) são resolvidas empregando-se o método utilizado por Arthurs [1], o qual considera o campo de velocidades $u^{n+1}$ e o campo de pressões $p^{n+1}$ 
como uma superposição de soluções, ou seja

$$
\begin{aligned}
& u^{n+1}=\bar{u}^{n+1}+\sum_{m=1}^{k} b_{m} Q_{m}^{n+1} \\
& p^{n+1}=\bar{p}^{n+1}+\sum_{m=1}^{k} r_{m} Q_{m}^{n+1}
\end{aligned}
$$

onde

$$
\left(\bar{p}^{n+1}, \bar{u}^{n+1}\right)
$$

é solução das equações

$$
\begin{aligned}
& G \bar{p}^{n+1}=\frac{\rho}{\Delta t}\left(u^{n+1,2}-\bar{u}^{n+1}\right) \\
& D \cdot \bar{u}^{n+1}=0
\end{aligned}
$$

e $b_{m}$ e $r_{m}$ são funções, vetorial e escalar respectivamente, definidas na malha e que devem ser calculadas.

Aplicando-se o operador divergente (3.13) à equação (3.33) e empregando-se a equação (3.34), obtém-se:

$$
\begin{gathered}
D . G \bar{p}^{n+1}=\frac{\rho}{\Delta t}\left(D \cdot u^{n+1,2}-D \cdot \bar{u}^{n+1}\right) \\
D \cdot G \bar{p}^{n+1}=\frac{\rho}{\Delta t} D \cdot u^{n+1,2}
\end{gathered}
$$

A equação (3.35) caracteriza uma equação de Poisson para o campo escalar $\bar{p}^{n+1}$, a qual será resolvida, assim como a equação (3.26), através da transformada rápida de Fourier (FFT). Calculado o campo $\bar{p}^{n+1}$, pode-se retornar à equação (3.33) para calcular o campo de velocidades $\bar{u}^{n+1}$.

Apesar de se ter calculado $\left(\bar{p}^{n+1}, \bar{u}^{n+1}\right)$, ainda não se pode determinar $u^{n+1}$, necessário à atualização da posição dos pontos que representam a fronteira imersa. Para tanto, precisa-se calcular as funções $b_{m}$ e $r_{m}$ e a vazão $Q_{m}$. 
Substituindo-se (3.31) e (3.32) em (3.29):

$$
\begin{aligned}
& G p^{n+1}=\frac{\rho}{\Delta t}\left(u^{n+1,2}-u^{n+1}\right) \\
& G\left(\bar{p}^{n+1}+\sum_{m=1}^{k} r_{m} Q_{m}^{n+1}\right)=\frac{\rho}{\Delta t}\left(u^{n+1,2}-\bar{u}^{n+1}-\sum_{m=1}^{k} b_{m} Q_{m}^{n+1}\right) \\
& G \bar{p}^{n+1}+\sum_{m=1}^{k} G r_{m} Q_{m}^{n+1}=\frac{\rho}{\Delta t} u^{n+1,2}-\frac{\rho}{\Delta t} \bar{u}^{n+1}-\frac{\rho}{\Delta t} \sum_{m=1}^{k} b_{m} Q_{m}^{n+1} \\
& \frac{\rho}{\Delta t} \bar{u}^{n+1}+\frac{\rho}{\Delta t} \sum_{m=1}^{k} b_{m} Q_{m}^{n+1}-\frac{\rho}{\Delta t} u^{n+1,2}=-G \bar{p}^{n+1}-\sum_{m=1}^{k} G r_{m} Q_{m}^{n+1}
\end{aligned}
$$

Aplicando-se (3.33) à equação (3.36):

$$
\begin{gathered}
\frac{\rho}{\Delta t} \bar{u}^{n+1}+\frac{\rho}{\Delta t} \sum_{m=1}^{k} b_{m} Q_{m}^{n+1}-\frac{\rho}{\Delta t} u^{n+1,2}=-\frac{\rho}{\Delta t} u^{n+1,2}+\frac{\rho}{\Delta t} \bar{u}^{n+1}-\sum_{m=1}^{k} G r_{m} Q_{m}^{n+1} \\
\frac{\rho}{\Delta t} \sum_{m=1}^{k} b_{m} Q_{m}^{n+1}+\sum_{m=1}^{k} G r_{m} Q_{m}^{n+1}=0 \\
\sum_{m=1}^{k}\left(\frac{\rho}{\Delta t} b_{m}+G r_{m}\right) Q_{m}^{n+1}=0
\end{gathered}
$$

Como a vazão $Q$ não é nula para cada $m$, uma vez que vazão nula implicaria em não considerar a m-ésima fonte e o m-ésimo sorvedouro a ela associados, então conclui-se da equação (3.37) que:

$$
\begin{gathered}
\frac{\rho}{\Delta t} b_{m}+G r_{m}=0 \quad \forall m \\
\frac{\rho}{\Delta t} b_{m}=-G r_{m}
\end{gathered}
$$

A equação (3.38) relaciona a função vetorial $b_{m}$ e a função escalar $r_{m}$. Então, uma vez calculada uma delas, pode-se calcular a outra.

Substituindo-se a equação (3.31) na equação (3.30):

$$
\begin{aligned}
& D . u^{n+1}=\sum_{m=1}^{k} Q_{m}^{n+1} \Psi_{m} \\
& D .\left(\bar{u}^{n+1}+\sum_{m=1}^{k} b_{m} Q_{m}^{n+1}\right)=\sum_{m=1}^{k} Q_{m}^{n+1} \Psi_{m} \\
& D . \bar{u}^{n+1}+\sum_{m=1}^{k} D \cdot\left(b_{m} Q_{m}^{n+1}\right)=\sum_{m=1}^{k} Q_{m}^{n+1} \Psi_{m}
\end{aligned}
$$


Empregando-se a equação (3.34) chega-se a:

$$
\begin{gathered}
\sum_{m=1}^{k} D \cdot\left(b_{m} Q_{m}^{n+1}\right)=\sum_{m=1}^{k} Q_{m}^{n+1} \Psi_{m} \\
\sum_{m=1}^{k}\left(D \cdot b_{m}-\Psi_{m}\right) Q_{m}^{n+1}=0
\end{gathered}
$$

Novamente, a equação (3.39) é independente da vazão. Assim:

$$
\begin{gathered}
D . b_{m}-\Psi_{m}=0 \quad \forall m \\
D . b_{m}=\Psi_{m}
\end{gathered}
$$

Na equação (3.40), $\Psi_{m}$ denota a distribuição espacial da m-ésima fonte e do m-ésimo sorvedouro e é conhecida. A Figura 3.5 ilustra a função $\Psi_{m}(x)$ para $k=3$ e $h_{o}=\frac{1}{32}$. As fontes têm centro em $(0.3,0.2),(0.3,0.5)$ e $(0.3,0.8)$, e os sorvedouros em $(0.7,0.2)$, $(0.7,0.5)$ e $(0.7,0.8)$.

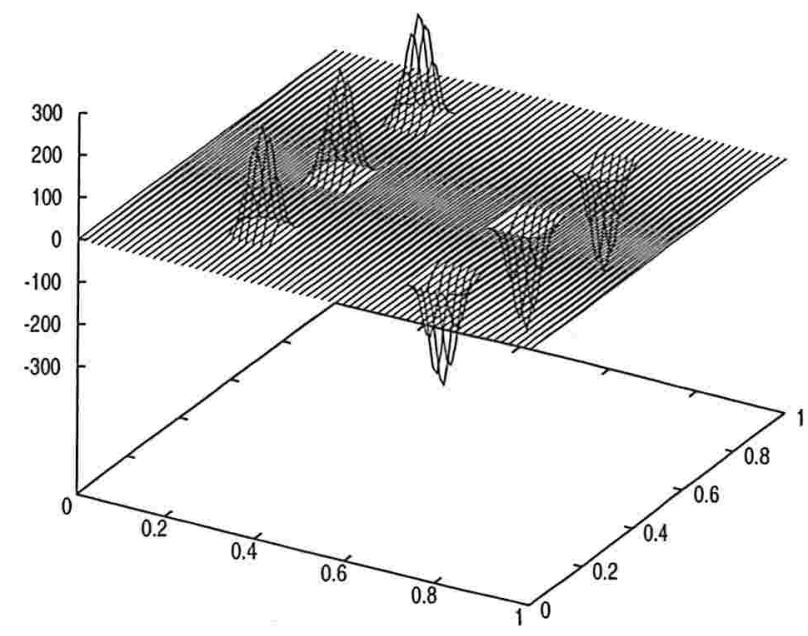

Figura 3.5: Função $\Psi_{m}(x)$ para três fontes e três sorvedouros 
Aplicando-se agora o operador divergente (3.13) à equação (3.38):

$$
\begin{aligned}
& \frac{\rho}{\Delta t} D \cdot b_{m}+D \cdot G r_{m}=0 \\
& D \cdot G r_{m}=-\frac{\rho}{\Delta t} D \cdot b_{m}
\end{aligned}
$$

Substituindo-se a expressão (3.40) na equação acima:

$$
D . G r_{m}=-\frac{\rho}{\Delta t} \Psi_{m}
$$

A equação (3.41) constitui uma equação de Poisson para o campo escalar $r_{m}$, a qual novamente é solucionada com o emprego da FFT.

Determinando o campo escalar $r_{m}$, pode-se então voltar à equação (3.38) para calcular o campo vetorial $b_{m}$.

Diferentemente da solução $\left(\bar{p}^{n+1}, \bar{u}^{n+1}\right)$, que dever ser encontrada por intermédio de uma equação de Poisson a cada passo no tempo, as funções $b_{m}$ e $r_{m}$ são calculadas através de uma equação de Poisson uma única vez, pois são independentes do tempo.

Para determinar $u^{n+1}$ resta ainda calcular a vazão $Q_{m}$ associada a cada par de fonte e sorvedouro.

Discretizando-se a expressão (2.29) e empregando (3.32) tem-se que:

$$
\begin{aligned}
& \sum_{l, s}\left(\bar{p}^{n+1}+\sum_{i=1}^{k} r_{i} Q_{i}^{n+1}\right) \Psi_{m} h^{2}=\bar{P}_{m}-R_{m} Q_{m}^{n+1} \\
& \sum_{l, s} \bar{p}^{n+1} \Psi_{m} h^{2}+\sum_{i=1}^{k}\left(\sum_{\Omega_{h}} r_{i} \Psi_{m} h^{2}\right) Q_{i}^{n+1}=\bar{P}_{m}-R_{m} Q_{m}^{n+1} \\
& \left\langle\bar{p}^{n+1}, \Psi_{m}\right\rangle_{h}+\sum_{i=1}^{k}\left\langle r_{i}(x), \Psi_{m}(x)\right\rangle_{h} Q_{i}^{n+1}=\bar{P}_{m}-R_{m} Q_{m}^{n+1} \\
& \sum_{i=1}^{k}\left\langle r_{i}(x), \Psi_{m}(x)\right\rangle_{h} Q_{i}^{n+1}+R_{m} Q_{m}^{n+1}=\bar{P}_{m}-\left\langle\bar{p}^{n+1}, \Psi_{m}\right\rangle_{h}
\end{aligned}
$$

Como $m=1, \ldots, k$ e $\left\langle r_{i}, \Psi_{m}\right\rangle_{h} \approx\left\langle r_{i}, \Psi_{m}\right\rangle=\int_{\Omega} r_{i}(x) \Psi_{m}(x) d x$, então para: 
$m=1$

$$
\begin{aligned}
& \sum_{i=1}^{k}\left\langle r_{i}, \Psi_{1}\right\rangle_{h} Q_{i}^{n+1}+R_{1} Q_{1}^{n+1}=\bar{P}_{1}-\left\langle\bar{p}^{n+1}, \Psi_{1}\right\rangle_{h} \\
& \left\langle r_{1}, \Psi_{1}\right\rangle_{h} Q_{1}^{n+1}+\left\langle r_{2}, \Psi_{1}\right\rangle_{h} Q_{2}^{n+1}+\ldots+\left\langle r_{k}, \Psi_{1}\right\rangle_{h} Q_{k}^{n+1}+R_{1} Q_{1}^{n+1}=\bar{P}_{1}-\left\langle\bar{p}^{n+1}, \Psi_{1}\right\rangle_{h} \\
& \left(\left\langle r_{1}, \Psi_{1}\right\rangle_{h}+R_{1}\right) Q_{1}^{n+1}+\left\langle r_{2}, \Psi_{1}\right\rangle_{h} Q_{2}^{n+1}+\ldots+\left\langle r_{k}, \Psi_{1}\right\rangle_{h} Q_{k}^{n+1}=\bar{P}_{1}-\left\langle\bar{p}^{n+1}, \Psi_{1}\right\rangle_{h}
\end{aligned}
$$

$m=2$

$$
\begin{aligned}
& \sum_{i=1}^{k}\left\langle r_{i}, \Psi_{2}\right\rangle_{h} Q_{i}^{n+1}+R_{2} Q_{2}^{n+1}=\bar{P}_{2}-\left\langle\bar{p}^{n+1}, \Psi_{2}\right\rangle_{h} \\
& \left\langle r_{1}, \Psi_{2}\right\rangle_{h} Q_{1}^{n+1}+\left\langle r_{2}, \Psi_{2}\right\rangle_{h} Q_{2}^{n+1}+\ldots+\left\langle r_{k}, \Psi_{2}\right\rangle_{h} Q_{k}^{n+1}+R_{2} Q_{2}^{n+1}=\bar{P}_{2}-\left\langle\bar{p}^{n+1}, \Psi_{2}\right\rangle_{h} \\
& \left\langle r_{1}, \Psi_{2}\right\rangle_{h} Q_{1}^{n+1}+\left(\left\langle r_{2}, \Psi_{2}\right\rangle_{h}+R_{2}\right) Q_{2}^{n+1}+\ldots+\left\langle r_{k}, \Psi_{2}\right\rangle_{h} Q_{k}^{n+1}=\bar{P}_{2}-\left\langle\bar{p}^{n+1}, \Psi_{2}\right\rangle_{h}
\end{aligned}
$$

$m=k$

$$
\begin{aligned}
& \sum_{i=1}^{k}\left\langle r_{i}, \Psi_{k}\right\rangle_{h} Q_{i}^{n+1}+R_{k} Q_{k}^{n+1}=\bar{P}_{k}-\left\langle\bar{p}^{n+1}, \Psi_{k}\right\rangle_{h} \\
& \left\langle r_{1}, \Psi_{k}\right\rangle_{h} Q_{1}^{n+1}+\left\langle r_{2}, \Psi_{k}\right\rangle_{h} Q_{2}^{n+1}+\ldots+\left\langle r_{k}, \Psi_{k}\right\rangle_{h} Q_{k}^{n+1}+R_{k} Q_{k}^{n+1}=\bar{P}_{k}-\left\langle\bar{p}^{n+1}, \Psi_{k}\right\rangle_{h} \\
& \left\langle r_{1}, \Psi_{k}\right\rangle_{h} Q_{1}^{n+1}+\left\langle r_{2}, \Psi_{k}\right\rangle_{h} Q_{2}^{n+1}+\ldots+\left(\left\langle r_{k}, \Psi_{k}\right\rangle_{h}+R_{k}\right) Q_{k}^{n+1}=\bar{P}_{k}-\left\langle\bar{p}^{n+1}, \Psi_{k}\right\rangle_{h}
\end{aligned}
$$

Logo, para determinar a vazão $Q$ associada a cada par de fonte e sorvedouro precisa-se solucionar, a cada passo no tempo, o sistema de equações lineares 


$$
A Q^{n+1}=B^{n+1}
$$

com

$$
A=\left[\begin{array}{lllll}
\left\langle r_{1}, \Psi_{1}\right\rangle_{h}+R_{1} & \left\langle r_{2}, \Psi_{1}\right\rangle_{h} & \cdots & \left\langle r_{k-1}, \Psi_{1}\right\rangle_{h} & \left\langle r_{k}, \Psi_{1}\right\rangle_{h} \\
\left\langle r_{1}, \Psi_{2}\right\rangle_{h} & \left\langle r_{2}, \Psi_{2}\right\rangle_{h}+R_{2} & \cdots & \left\langle r_{k-1}, \Psi_{2}\right\rangle_{h} & \left\langle r_{k}, \Psi_{2}\right\rangle_{h} \\
\vdots & \vdots & \vdots & \vdots & \vdots \\
\left\langle r_{1}, \Psi_{k-1}\right\rangle_{h} & \left\langle r_{2}, \Psi_{k-1}\right\rangle_{h} & \cdots & \left\langle r_{k-1}, \Psi_{k-1}\right\rangle_{h}+R_{k-1} & \left\langle r_{k}, \Psi_{k-1}\right\rangle_{h} \\
\left\langle r_{1}, \Psi_{k}\right\rangle_{h} & \left\langle r_{2}, \Psi_{k}\right\rangle_{h} & \cdots & \left\langle r_{k-1}, \Psi_{k}\right\rangle_{h} & \left\langle r_{k}, \Psi_{k}\right\rangle_{h}+R_{k}
\end{array}\right]
$$

$$
\begin{gathered}
Q^{n+1}=\left[\begin{array}{c}
Q_{1}^{n+1} \\
Q_{2}^{n+1} \\
\vdots \\
Q_{k-1}^{n+1} \\
Q_{k}^{n+1}
\end{array}\right] \\
B^{n+1}=\left[\begin{array}{l}
\bar{P}_{1}-\left\langle\bar{p}^{n+1}, \Psi_{1}\right\rangle_{h} \\
\bar{P}_{2}-\left\langle\bar{p}^{n+1}, \Psi_{2}\right\rangle_{h} \\
\vdots \\
\bar{P}_{k-1}-\left\langle\bar{p}^{n+1}, \Psi_{k-1}\right\rangle_{h} \\
\bar{P}_{k}-\left\langle\bar{p}^{n+1}, \Psi_{k}\right\rangle_{h}
\end{array}\right]
\end{gathered}
$$

A matriz A é calculada uma única vez, pois independe do tempo. Já as matrizes Q e $\mathrm{B}$ são atualizadas a cada passo no tempo.

Tomando-se $\bar{a}_{i, j}=\left\langle r_{j}, \Psi_{i}\right\rangle_{h}$, com $i, j=1, \ldots, k$, a matriz A (3.43) pode ser reescrita 
como:

$$
A=\left[\begin{array}{lllll}
\bar{a}_{1,1}+R_{1} & \bar{a}_{1,2} & \cdots & \bar{a}_{1, k-1} & \bar{a}_{1, k} \\
\bar{a}_{2,1} & \bar{a}_{2,2}+R_{2} & \cdots & \bar{a}_{2, k-1} & \bar{a}_{2, k} \\
\vdots & \vdots & \vdots & \vdots & \vdots \\
\bar{a}_{k-1,1} & \bar{a}_{k-1,2} & \cdots & \bar{a}_{k-1, k-1}+R_{k-1} & \bar{a}_{k-1, k} \\
\bar{a}_{k, 1} & \bar{a}_{k, 2} & \cdots & \bar{a}_{k, k-1} & \bar{a}_{k, k}+R_{k}
\end{array}\right]
$$

Pode-se mostrar que a matriz $A(3.43)$ é simétrica. Os elementos da mesma são dados por

$$
\begin{array}{ll}
a_{i j}=\left\langle r_{j}, \Psi_{k}\right\rangle_{h} & \text { se } i \neq j \\
a_{i j}=\left\langle r_{j}, \Psi_{k}\right\rangle_{h}+\delta_{j k} R_{k} & \text { se } i=j
\end{array}
$$

$\operatorname{com} \delta_{j k}= \begin{cases}1, & \text { se } j=k \\ 0, & \text { se } j \neq k\end{cases}$

Aplicando-se o operador divergente à equação (3.38)

$$
\frac{\rho}{\Delta t} b_{m}=-G r_{m}
$$

tem-se que:

$$
\frac{\rho}{\Delta t} D \cdot b_{m}=-D \cdot G r_{m}
$$

Substituindo-se na equação anterior a equaçao (3.40)

$$
D \cdot b_{m}=\Psi_{m}
$$

chega-se a:

$$
\begin{aligned}
& \frac{\rho}{\Delta t} \Psi_{m}=-D \cdot G r_{m} \\
& \Psi_{m}=-\frac{\Delta t}{\rho} D \cdot G r_{m}
\end{aligned}
$$


Empregando-se a equivalência anterior no produto interno $\left\langle r_{j}, \Psi_{k}\right\rangle_{h}$ que define os elementos não pertencentes à diagonal principal da matriz $A$, conclui-se que:

$$
\begin{aligned}
\left\langle r_{j}, \Psi_{k}\right\rangle_{h} & = \\
\left\langle r_{j},-\frac{\Delta t}{\rho} D \cdot G r_{k}\right\rangle_{h} & = \\
-\frac{\Delta t}{\rho}\left\langle r_{j}, D \cdot G r_{k}\right\rangle_{h} & = \\
-\frac{\Delta t}{\rho}\left\langle D \cdot G r_{j}, r_{k}\right\rangle_{h} & =(*) \\
-\frac{\Delta t}{\rho}\left\langle r_{k}, D \cdot G r_{j}\right\rangle_{h} & = \\
\left\langle r_{k},-\frac{\Delta t}{\rho} D \cdot G r_{j}\right\rangle_{h} & = \\
\left\langle r_{k}, \Psi_{j}\right\rangle_{h} &
\end{aligned}
$$

$\left({ }^{*}\right)$ Peskin [25]

Como a resolução do sistema linear $A Q^{n+1}=B^{n+1}$ fornece a vazão associada a toda fonte e sorvedouro em cada instante de tempo, pode-se agora retornar à equação (3.31) e determinar a velocidade associada a cada ponto do fluido, a qual será posteriormente empregada na equação (3.28) para atualizar a posição dos pontos da fibra imersa, completando assim um passo no tempo.

A introdução de fontes e sorvedouros modifica o algoritmo anteriormente apresentado para o método da fronteira imersa. Estrutura-se a seguir um algoritmo para o método que incorpora essas alterações.

\subsection{Algoritmo para introdução de fontes e sorvedou- ros}

O algoritmo estruturado a seguir possibilita uma melhor compreensão das etapas a serem seguidas na execuçao do método da fronteira imersa com a inclusão de $k$ fontes e $k$ sorvedouros. 


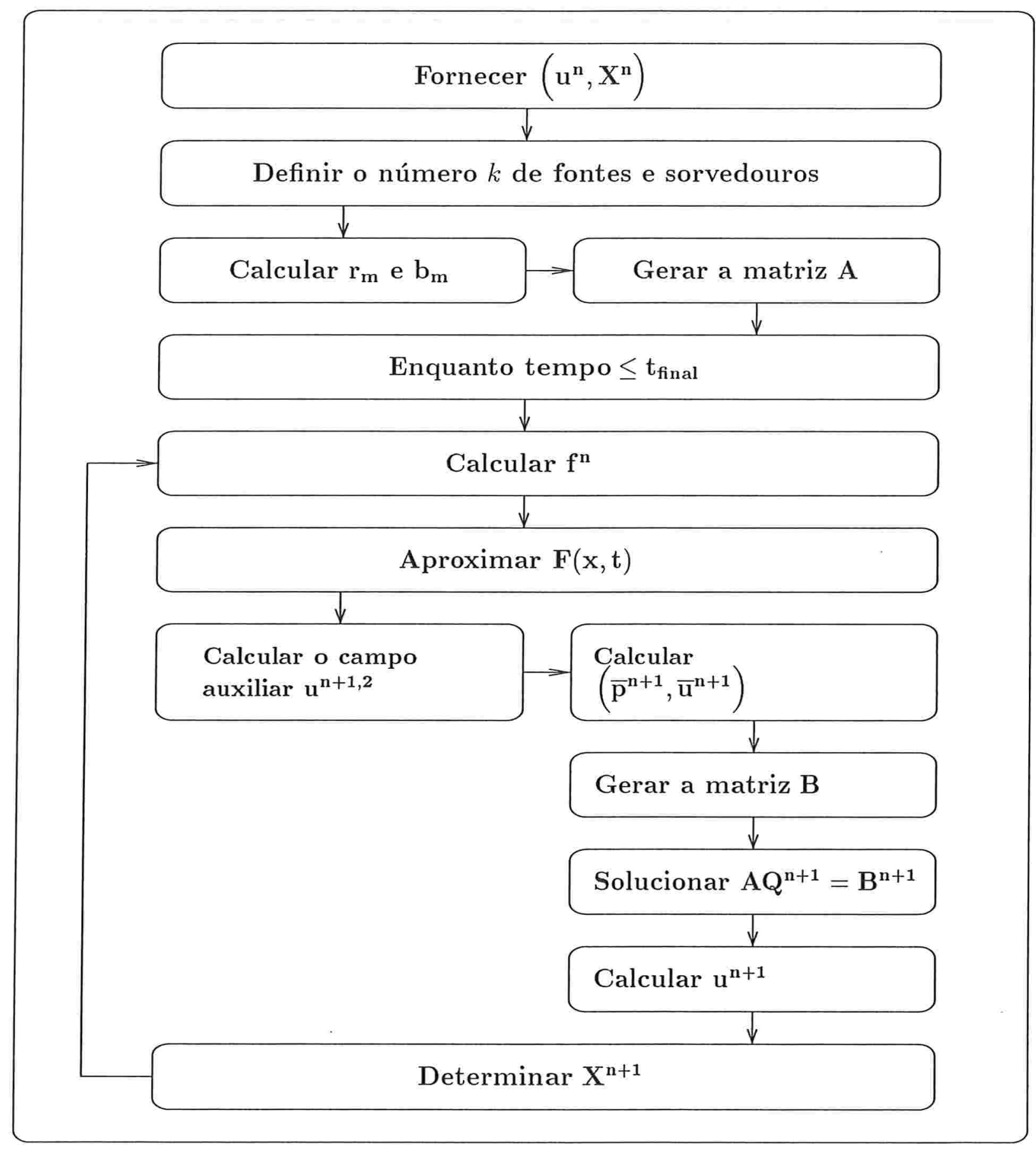

Figura 3.6: Algoritmo estruturado do método da fronteira imersa com a inclusão de $k$ fontes e $k$ sorvedouros 
Objetiva-se calcular $\left(u^{n+1}, X^{n+1}\right)$ a partir de $\left(u^{n}, X^{n}\right)$. Isto é feito da seguinte maneira:

1ㅇ passo:

- Calcular o campo escalar $r_{m}$ e o campo vetorial $b_{m}$ por intermédio das equações (3.41) e (3.38), respectivamente;

- Gerar a matriz A (3.43).

$2^{\text {o }}$ passo: Calcular a densidade de força elástica $f^{n}$.

3ำ passo: Distribuir a densidade de força elástica exercida pela fronteira imersa aos pontos do fluido por intermédio da equação (3.27).

4 passo: Solucionar as equações de Navier-Stokes empregando uma variação do método de projeção de Chorin.

1. etapa: Calcular o campo de velocidades auxiliar $u^{n+1,2}$ através das equações (3.18), (3.19) e (3.20). As equações (3.19) e (3.20) geram sistemas tridiagonais periódicos, os quais são solucionados através do algoritmo de Thomas.

$2^{\text {a }}$ etapa:

- Calcular $\left(\bar{p}^{n+1}, \bar{u}^{n+1}\right)$, solução das equações (3.33) e (3.34). Estas equações geram uma Poisson - equação (3.35) - para o campo escalar $\bar{p}^{n+1}$;

- Gerar a matriz B (3.44);

- Determinar as vazões associadas a cada fonte e sorvedouro solucionando o sistema de equações lineares (3.42);

- Calcular $u^{n+1}$ através da equação (3.31).

5o passo: Atualizar a posição dos pontos da fronteira imersa usando a equação (3.28). 


\subsection{Força exercida pela fronteira}

No modelo matemático bidimensional adotado para um alvéolo - Figura 3.7, empregouse para os pontos dos segmentos $\overline{A B}, \overline{A C}$ e $\overline{C D}$ uma força do tipo restauradora - equação (2.30). Utilizou-se $\mathrm{k}=10^{6} \frac{\mathrm{dyn}}{\mathrm{cm}}$ com o intuito de manter essas partes da fronteira rígidas.

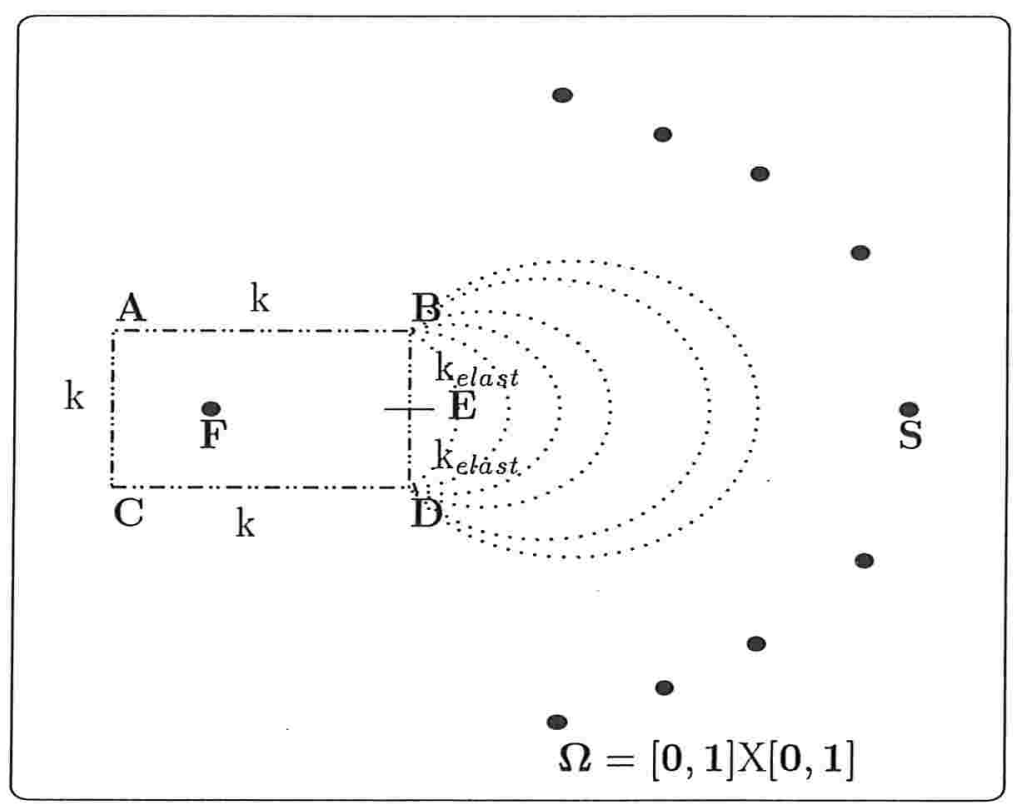

Figura 3.7: Modelo para um alvéolo pulmonar

No segmento $\overline{B D}$, com $y_{E}=\frac{y_{B}+y_{D}}{2}$, adotou-se o modelo usado por Roma [42] para a densidade de força elástica, isto é

$$
f(i)=\frac{\mathrm{k}_{\text {elast }}}{\Delta s^{2}}[X(i+1)+X(i-1)-2 . X(i)]
$$

onde $X$ representa a posição dos pontos da fronteira imersa e $\Delta s$ a distância entre dois pontos consecutivos da mesma.

A expressão (3.46) provém da discretização da lei generalizada de Hooke - equação (2.6)

$$
T(s, t)=T_{o}\left\|\frac{\partial X}{\partial s}(s, t)\right\|
$$

a qual fornece a tensão na fibra.

A equação (3.47) indica que a tensão é proporcional ao valor da constante $T_{0}$. Substituindose a expressão (3.47) na equação (2.8), conclui-se que a força exercida pela fronteira é 
modelada por

$$
f(s, t)=T_{o} \frac{\partial^{2} X}{\partial s^{2}}(s, t)
$$

Como se emprega $D_{x}^{+} D_{x}^{-}$para aproximar o operador $\frac{\partial^{2}}{\partial x^{2}}$ (derivada segunda), então a densidade de força elástica $f(s, t)$ dada pela equação (3.48) é discretizada por

$$
f_{l}^{n}=T_{o} D_{s}^{+} D_{s}^{-} X_{l}^{n}
$$

Aplicando-se as aproximações para a primeira derivada regressiva - equação (3.9) - e a primeira derivada progressiva - equação (3.6) - chega-se a:

$$
\begin{aligned}
f_{l}^{n} & =T_{o} D_{s}^{+}\left[\frac{X_{l}^{n}-X_{l-1}^{n}}{\Delta s}\right] \\
f_{l}^{n} & =\frac{T_{o}}{\Delta s}\left[\frac{X_{l+1}^{n}-X_{l}^{n}}{\Delta s}-\frac{X_{l}^{n}-X_{l-1}^{n}}{\Delta s}\right] \\
f_{l}^{n} & =\frac{T_{o}}{\Delta s^{2}}\left[X_{l+1}^{n}+X_{l-1}^{n}-2 \cdot X_{l}^{n}\right]
\end{aligned}
$$

onde $T_{o}=\mathrm{k}_{\text {elast }} \geq 0$.

A discretização dada para a densidade de força elástica pela equação (3.49) permite modelar uma fibra flexível. Segundo Peskin [24], uma membrana "infinitamente" flexível é modelada através da articulação de pontos sucessivos da mesma. Já uma estrutura "quase" rígida é modelada pela articulação dos pontos de sua fronteira com pontos fixos no domínio espacial. Neste caso, a rigidez será conferida pelo emprego de grandes valores para a constante de proporcionalidade na equação (2.30).

O uso de uma função que modifica a "elasticidade" $\mathrm{k}_{\text {elast }}=T_{0}$ ao longo do segmento $\overline{B D}$ no decorrer do tempo, permite modelar a variação de parâmetros fisiológicos tais como o surfactante, o qual altera a elasticidade da parede alveolar durante a inspiração. $\mathrm{O}$ mesmo não acontece quando se atribui um valor constante à elasticidade associada aos pontos do segmento $\overline{B D}$.

Dessa forma, atribui-se aos pontos do segmento $\overline{B E}$

$$
k[i]=X(i)
$$

onde $k[i]=\mathrm{k}_{\text {elast }}=T_{o}$ é o valor da elasticidade associada a cada ponto do segmento e $X(i)$ é a ordenada do ponto na configuração inicial da fronteira $(t=0 s)$. Para se obter 
uma expansão simétrica dos segmentos $\overline{B E}$ e $\overline{D E}$, o valor da elasticidade associada a cada ponto do segmento $\overline{D E}$ foi calculada por

$$
k[i]=1-X(i)
$$

Além de se associar um valor distinto à elasticidade em cada ponto do segmento que simula a membrana alveolar, fez-se também esse valor variar no decorrer do tempo. A primeira função pensada para tal foi

$$
k^{*}[i]=e^{k[i] . n \Delta t}
$$

com $k[i]$ representando o valor da elasticidade associada a cada ponto na configuração inicial da fronteira e $n \Delta t$ o passo temporal computado em $n$ passos.

Com a adoção dessa função para a variação temporal, associa-se aos pontos mais extremos do segmento $\overline{B D}$ valores mais elevados para a elasticidade, o que induz uma menor expansão destes pontos, e valores mais baixos para a elasticidade nos pontos mais centrais do segmento, o que deixa esses pontos "mais soltos" e permite uma maior expansão dos mesmos.

Os valores iniciais atribuídos à elasticidade dos pontos $P$ do segmento $\overline{B D}$ podem ser alterados. Por exemplo, ao se adotar

$$
\begin{array}{ll}
k[i]=2 . X(i) & \text { se } P \in \overline{B E} \\
k[i]=2 .(1-X(i)) & \text { se } P \in \overline{D E}
\end{array}
$$

atribui-se valores iniciais mais elevados à elasticidade, o que implica em menor expansão da fronteira elástica. 


\section{Modelagem da fronteira elástica}

Para modelar a expansão da parede alveolar considerou-se:

- a inserção de um número adequado de fontes e sorvedouros, o posicionamento destes no domínio computacional e a pressão e a resistência associadas aos mesmos;

- a variação temporal dos valores atribuídos à elasticidade nos pontos da porção da fronteira imersa que avança com o fluido no decorrer do tempo. Esses valores são utilizados no cálculo da força exercida pela fronteira sobre as partículas do fluido próximas a ela.

A vazão $Q_{m}$ relacionada à m-ésima fonte e ao m-ésimo sorvedouro introduzidos no domínio de estudo da interação fluido-fronteira é necessária à resolução das equações que regem o escoamento do fluido.

$$
\begin{aligned}
& \rho\left(\frac{\partial u}{\partial t}+u . \nabla u\right)=-\nabla p+\mu \Delta u+F \\
& \nabla . u=q(x, t)=\sum_{m=1}^{k} Q_{m}(t) \Psi_{m}(x)
\end{aligned}
$$

A discretização dessas equações gera um sistema de equações lineares para o cálculo da vazão $Q$ presente na equação (4.2).

A força $F$ exercida pela fronteira imersa consta na equação (4.1) e a sua discretização é dada por

$$
F_{i, j}^{n}=\sum_{l} f_{l}^{n} \delta_{h_{o}}\left(x_{i, j}-X_{l}^{n}\right) \Delta s
$$

Na equação (4.3), a densidade de força elástica $f$ é discretizada por

$$
f_{l}^{n}=\frac{T_{o}}{\Delta s^{2}}\left[X_{l+1}^{n}+X_{l-1}^{n}-2 . X_{l}^{n}\right]
$$


onde $T_{o}=\mathrm{k}_{\text {elast }}$ é uma constante elástica. Diferentemente de outros trabalhos, não se considera neste $T_{o}=\mathrm{k}_{\text {elast }}$ constante. Uma função é estruturada para atribuir diferentes valores a ela no decorrer do tempo. Assim, a densidade de força elástica $f_{l}$ associada a um ponto $X_{l}$ da fronteira não varia tão somente com sua posição e a dos vizinhos $X_{l+1} \mathrm{e}$ $X_{l-1}$. A elasticidade associada a esse ponto também passa a determinar a força exercida por ele.

\subsection{Fontes e sorvedouros}

A inserção de fontes e sorvedouros modela o fluxo de um fluido no domínio computacional. A velocidade das partículas do fluido injetado é controlada pelos valores atribuídos à pressão $\bar{P}_{m}$ e à resistência $R_{m}$ associadas à m-ésima fonte e ao m-ésimo sorvedouro.

Implementou-se assim um código computacional em linguagem $\mathrm{C}$ que associa a cada fonte um sorvedouro e que permite a inserção de um número de fontes e sorvedouros $k \geq 2$. Para $k=1$ a função $q(x, t)$ - equação (4.2) - que modela a ação das fontes e dos sorvedouros tem outra estrutura. A pressão $P$ e a resistência $R$ associadas a cada fonte e sorvedouro foram mantidas constantes, isto é, $\bar{P}_{1}=\bar{P}_{2}=\ldots=\bar{P}_{m}$ e $R_{1}=R_{2}=\ldots=R_{m}$.

Para a expansão do alvéolo pulmonar durante a inspiração, os valores fisiológicos da pressão $\bar{P}_{m}$ e da resistência $R_{m}$ não são conhecidos. Recorreu-se então a parâmetros adotados em outros modelos para estabelecer valores iniciais às mesmas.

Empregou-se

$$
\begin{aligned}
& \bar{P}_{m}=10^{3} \frac{\mathrm{g}}{\mathrm{cm} \cdot \mathrm{s}^{2}} \\
& R_{m}=10^{5} \frac{\mathrm{g}}{\mathrm{cm}^{3} \cdot \mathrm{s}}
\end{aligned}
$$

nas $k$ fontes e sorvedouros introduzidos no modelo. Para se chegar a esses valores, partiuse do trabalho desenvolvido por Peskin [25], o qual empregou para a vazão sangüínea no lado esquerdo do coração o modelo de resistência linear dado por

$$
Q(t)=Q_{s}-\alpha_{s} P_{\text {atrium }}(t)
$$

sendo $Q_{s}$ e $\alpha_{s}$ constantes e $P_{\text {atrium }}=P_{a}-P_{e}$, com $P_{a}=$ pressão na fonte e $P_{e}=$ pressão no sorvedouro. 
A vazão dada pela expressão (4.5) pode ser reescrita como

$$
Q(t)=Q_{s}-\alpha_{s} P_{F, S}(t)
$$

onde $P_{F, S}(t)$ é a pressão fornecida pela diferença entre a pressão na fonte e a pressão no sorvedouro.

A equação (4.6) é vista como uma forma alternativa de se escrever o modelo de resitência linear fornecido pela equação (2.21):

$$
Q=\frac{p_{1}-p_{2}}{R}=\frac{\Delta p}{R}
$$

Logo, comparando-se as equações

$$
\begin{aligned}
& Q(t)=Q_{s}-\alpha_{s} P_{F, S}(t) \\
& Q(t)=\frac{p_{1}-p_{2}}{R}=\frac{p_{1}}{R}-\frac{1}{R} p_{2}
\end{aligned}
$$

conclui-se que:

$$
\begin{gathered}
\alpha_{s}=\frac{1}{R} \\
Q_{s}=\frac{p_{1}}{R} \\
p_{2}=P_{F, S}(t)
\end{gathered}
$$

Remigio [29] empregou

$$
\begin{aligned}
& \alpha_{s}=10^{-5} \frac{\mathrm{cm}^{3} \cdot s}{\mathrm{~g}} \\
& Q_{s}=10^{-2} \frac{\mathrm{cm}^{2}}{\mathrm{~s}}
\end{aligned}
$$

ao analisar o comportamento do método da fronteira imersa em escoamentos incompressíveis bidimensionais com a introdução de uma fonte e de um sorvedouro.

Substituindo-se esses valores nas expressões (4.7) e (4.8), chega-se a:

$$
\begin{aligned}
& \alpha_{s}=\frac{1}{R} \Longrightarrow 10^{-5} \frac{\mathrm{cm}^{3} . s}{g}=\frac{1}{R} \\
& R=10^{5} \frac{\mathrm{g}}{\mathrm{cm}^{3} \cdot \mathrm{s}}
\end{aligned}
$$




$$
\begin{aligned}
& Q_{s}=\frac{p_{1}}{R} \Longrightarrow 10^{-2} \frac{\mathrm{cm}^{2}}{\mathrm{~s}}=\frac{p_{1}}{10^{5} \frac{\mathrm{g}}{\mathrm{cm}^{3} \cdot \mathrm{s}}} \\
& p_{1}=10^{-2} \frac{\mathrm{cm}^{2}}{\mathrm{~s}} \cdot 10^{5} \frac{\mathrm{g}}{\mathrm{cm}^{3} \cdot \mathrm{s}} \\
& p_{1}=10^{3} \frac{\mathrm{g}}{\mathrm{cm} \cdot \mathrm{s}^{2}}
\end{aligned}
$$

Os valores da resistência $R_{m}$ e da pressão $\bar{P}_{m}$ ajudam a compor o sistema de equações lineares

$$
A Q^{n+1}=B^{n+1}
$$

para o cálculo da vazão $Q$ associada às $k$ fontes e sorvedouros introduzidos no modelo a cada instante de tempo $\Delta t$. Nesse sistema.

$$
\begin{aligned}
A= & {\left[\begin{array}{lllll}
\left\langle r_{1}, \Psi_{1}\right\rangle_{h}+R_{1} & \left\langle r_{2}, \Psi_{1}\right\rangle_{h} & \cdots & \left\langle r_{k-1}, \Psi_{1}\right\rangle_{h} & \left\langle r_{k}, \Psi_{1}\right\rangle_{h} \\
\left\langle r_{1}, \Psi_{2}\right\rangle_{h} & \left\langle r_{2}, \Psi_{2}\right\rangle_{h}+R_{2} & \cdots & \left\langle r_{k-1}, \Psi_{2}\right\rangle_{h} & \left\langle r_{k}, \Psi_{2}\right\rangle_{h} \\
\vdots & \vdots & \vdots & \vdots & \vdots \\
\left\langle r_{1}, \Psi_{k-1}\right\rangle_{h} & \left\langle r_{2}, \Psi_{k-1}\right\rangle_{h} & \cdots & \left\langle r_{k-1}, \Psi_{k-1}\right\rangle_{h}+R_{k-1} & \left\langle r_{k}, \Psi_{k-1}\right\rangle_{h} \\
\left\langle r_{1}, \Psi_{k}\right\rangle_{h} & \left\langle r_{2}, \Psi_{k}\right\rangle_{h} & \cdots & \left\langle r_{k-1}, \Psi_{k}\right\rangle_{h} & \left\langle r_{k}, \Psi_{k}\right\rangle_{h}+R_{k}
\end{array}\right] } \\
B^{n+1}= & {\left[\begin{array}{l}
\bar{P}_{1}-\left\langle\bar{p}^{n+1}, \Psi_{1}\right\rangle_{h} \\
\bar{P}_{2}-\left\langle\bar{p}^{n+1}, \Psi_{2}\right\rangle_{h} \\
\vdots \\
\bar{P}_{k-1}-\left\langle\bar{p}^{n+1}, \Psi_{k-1}\right\rangle_{h} \\
\bar{P}_{k}-\left\langle\bar{p}^{n+1}, \Psi_{k}\right\rangle_{h}
\end{array}\right] }
\end{aligned}
$$

Como os elementos da matriz $A$ são dados pelo produto interno entre a função escalar $r_{m}$ e a função $\Psi_{m}$, o seu determinante varia com:

- o número $k$ de fontes e sorvedouros empregado no modelo. Cada par fonte/sorvedouro acrescentado ao mesmo aumenta em um a ordem da matriz $A$;

- o centro das fontes e sorvedouros e o suporte $h_{o}$ adotado para as funções $W_{F_{m}}$ e $W_{S_{m}}$ equação (2.20);

- o maior valor $\mathrm{k}$ atribuído à elasticidade da fronteira. Esse valor é usado no cálculo do passo temporal $\Delta t$ - equação (3.1); 
- o valor empregado para a constante $R_{m}$;

- a densidade $\rho$ do fluido $\left(\left\langle r_{j}, \Psi_{i}\right\rangle=\left\langle r_{j},-\frac{\Delta t}{\rho} D . G r_{i}\right\rangle=-\frac{\Delta t}{\rho}\left\langle r_{j}, D . G r_{i}\right\rangle\right)$;

- o passo espacial $h=\Delta x=\Delta y$.

Dessa forma, determinou-se inicialmente os coeficientes e o determinante da matriz $A$ para $h=\frac{1}{32}, \mathrm{k}_{\max }=10^{6} \frac{\mathrm{dyn}}{\mathrm{cm}}, \Delta t=\frac{\sqrt{3}}{6000} \mathrm{~s}, \rho=1 \frac{\mathrm{g}}{\mathrm{cm}^{3}}, h_{o}=\frac{1}{32}, R_{m}=10^{5} \frac{\mathrm{g}}{\mathrm{cm}^{3} \cdot \mathrm{s}} \mathrm{e}$ $k=2,3,5,7$ e 9 . A resistência $R_{m}$ foi mantida constante para as $k$ fontes e sorvedouros inseridos no domínio computacional. As fontes foram todas centradas no ponto $(0.2,0.5)$, introduzindo assim um fluxo contínuo no interior do tubo que simula o bronquíolo. Para $k=2$, os sorvedouros foram centrados em $(0.85,0.3)$ e $(0.85,0.7)$; para $k=3$ em $(0.85,0.3),(0.9,0.5)$ e $(0.85,0.7)$; para $k=5 \mathrm{em}(0.75,0.2),(0.85,0.3),(0.9,0.5),(0.85,0.7)$ e $(0.75,0.8) ;$ para $k=7 \mathrm{em}(0.65,0.15),(0.75,0.2),(0.85,0.3),(0.9,0.5),(0.85,0.7)$, $(0.75,0.8)$ e $(0.65,0.85)$; para $k=9$ em $(0.55,0.1),(0.65,0.15),(0.75,0.2),(0.85,0.3)$, $(0.9,0.5),(0.85,0.7),(0.75,0.8),(0.65,0.85)$ e $(0.55,0.9)$. Adotou-se estas posições para os sorvedouros pensando-se em simular uma expansão "menos alongada" da porção da fronteira imersa que simula o alvéolo pulmonar. Escolheu-se um número $k$ ímpar por este permitir uma distribuição simétrica dos sorvedouros em relação à reta $y=0.5$, eixo de simetria da configuração inicial utilizada para a fronteira imersa, incluindo um sorvedouro exatamente sobre ela.

Para os parâmetros escolhidos anteriormente, a matriz $A$ é estritamente diagonal dominante, devido ao alto valor empregado para a resistência $R_{m}$ presente em sua diagonal principal, e simétrica positiva definida. Logo, a matriz $A$ é não singular, fato que garante a existência e unicidade de solução do sistema linear

$$
A Q^{n+1}=B^{n+1}
$$

Constatou-se também que a inserção de um número $k$ crescente de fontes e sorvedouros possibilita maior expansão da parte elástica da fronteira imersa, como ilustra a Figura 4.1. Nas simulações presentes nesta figura, empregou-se

$$
k[i]=1.0
$$

para todos os pontos do segmento $\overline{B D}$, ou seja, a elasticidade $\mathrm{k}_{\text {elast }}=T_{o}$ associada a esses pontos foi mantida constante no decorrer do tempo, $\bar{P}_{m}=10^{3} \frac{\mathrm{g}}{\mathrm{cm} \cdot \mathrm{s}^{2}}$ e $\mu=10^{-2} \frac{\mathrm{g}}{\mathrm{cm} . \mathrm{s}}$. $\mathrm{O}$ primeiro quadro mostra a geometria inicial utilizada e os cinco demais representam, respectivamente as simulações para $k=2, k=3, k=5, k=7$ e $k=9$. 

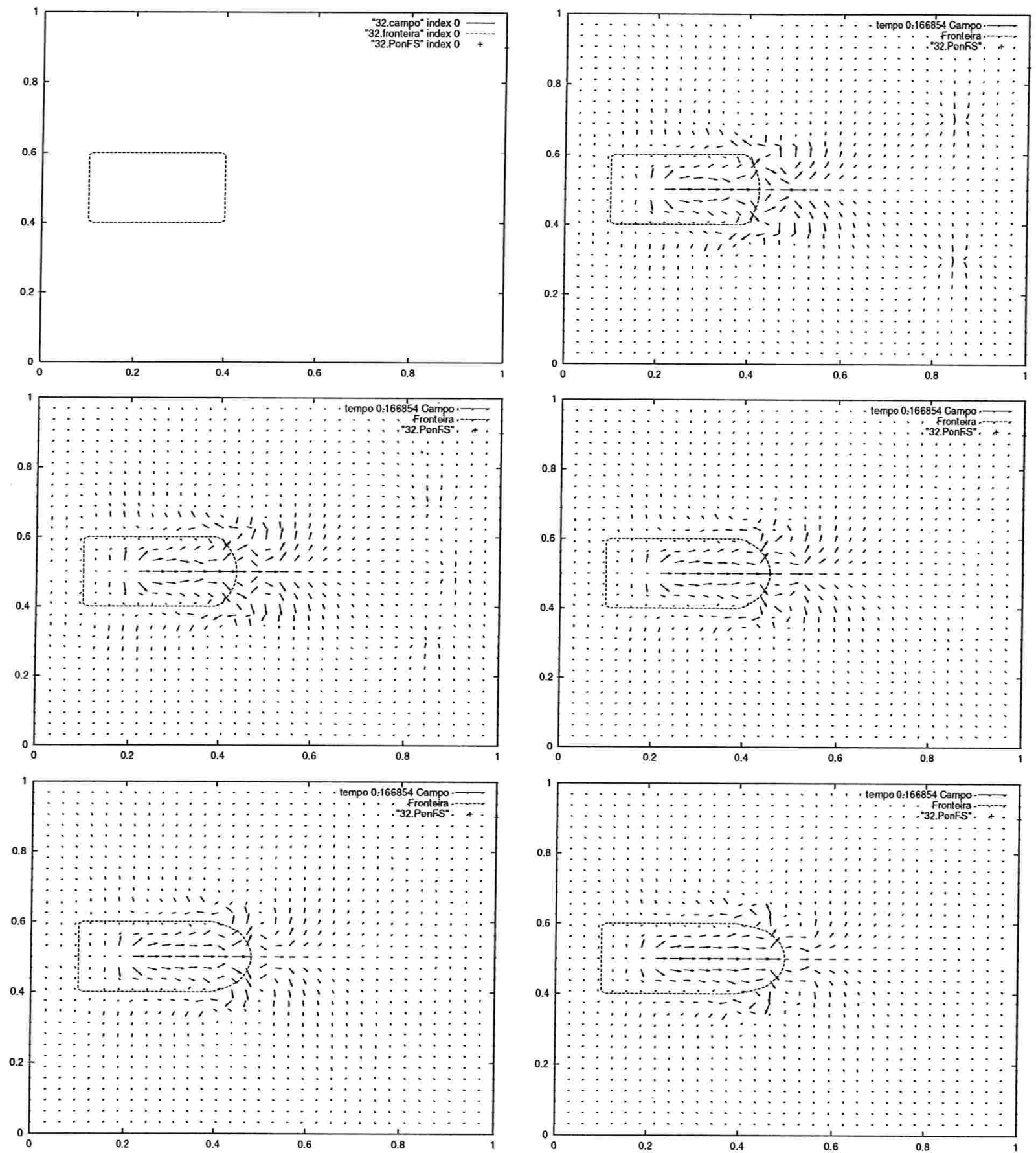

Figura 4.1: Comportamento da geometria inicial da fronteira imersa para $k=2,3,5,7$ e 9 após $t \approx 0.17 \mathrm{~s}$ 
A variação do parâmetro $k$ permite menor ou maior expansão da fibra elástica. Porém, os valores da pressão $\bar{P}_{m}$ e da resistência $R_{m}$ na m-ésima fonte e no m-ésimo sorvedouro, do suporte $\left[-2 h_{o}, 2 h_{o}\right]$ das funções $W_{F_{m}}$ e $W_{S_{m}}$, da densidade $\rho$ do fluido e da constante elástica $\mathrm{k}_{\text {elast }}=T_{o}$, também influenciam nessa expansão, assim como os demais parâmetros associados às matrizes $A$ e $B$ no sistema linear $A Q^{n+1}=B^{n+1}$, o campo inicial de velocidades $u^{n}$ e a viscosidade $\mu$ do fluido.

Assim, efetuou-se uma série de simulações para testar a correlação entre a pressão $\bar{P}_{m}$, a resistência $R_{m}$ e o suporte $h_{o}$ na expansão da fronteira imersa para um $k$ específico.

As tabelas presentes no Apêndice D trazem os resultados das simulações efetuadas para testar a associação da pressão $\bar{P}_{m}\left(\frac{g}{c m \cdot s^{2}}\right)$, da resistência $R_{m}\left(\frac{g}{\mathrm{~cm}^{3} \cdot s}\right)$ e do suporte $h_{o}$ na expansão da porção elástica da fronteira imersa com a introdução de cinco fontes e cinco sorvedouros no domínio computacional. Nesses testes se manteve o maior valor k da elasticidade igual a $10^{6} \frac{\mathrm{dyn}}{\mathrm{cm}}, \rho=1 \frac{\mathrm{g}}{\mathrm{cm}^{3}}, \mu=10^{-2} \frac{\mathrm{g}}{\mathrm{cm} . \mathrm{s}}, t_{\text {final }}=1.5 \mathrm{~s}$ e a geometria relatada no segundo capítulo. Para a elasticidade dos pontos do segmento $\overline{B D}$ empregou-se

$$
k[i]=1.0
$$

valor mantido constante no decorrer do tempo. Dessa forma, não se analisa ainda a influência da evolução temporal dos valores de $k_{\text {elast }}=T_{o}$ na expansão da fronteira imersa. Os testes foram realizados em uma malha computacional $32 \times 32$ e as fontes e os sorvedouros, associados um a um, foram centrados em $(0.2,0.5)$ e $(0.75,0.2),(0.2,0.5)$ e $(0.85,0.3)$, $(0.2,0.5)$ e $(0.9,0.5),(0.2,0.5)$ e $(0.85,0.7),(0.2,0.5)$ e $(0.75,0.8)$.

A partir desses testes, pode-se estabelecer uma relação entre as constantes $R_{m}$ e $\bar{P}_{m}$ para $k=5$ e $\mathrm{k}_{\text {elast }}=T_{\mathrm{o}}=1.0$.

Uma expansão uniforme e controlada da fronteira foi obtida para $R_{m}=10^{5} \frac{\mathrm{g}}{\mathrm{cm}^{3} \cdot \mathrm{s}}$ e $\bar{P}_{m}=10^{3} \frac{g}{c m . s^{2}}$, exatamente os valores calculados a partir do modelo de resistência linear para a vazão fornecido pela lei de Hagen-Poiseuille - equação (A.1). Essa diferença adimensional de cerca de $10^{5}$ entre as constantes $R_{m}$ e $\bar{P}_{m}$, com $R_{m}>\bar{P}_{m}$, mantém geralmente os resultados satisfatórios para todos os suportes testados quando se considera

$$
10^{2} \leq \bar{P}_{m} \leq 10^{4}
$$

A figura 4.2 ilustra a expansão homogênea da fronteira verificada quando essas condições são respeitadas. 

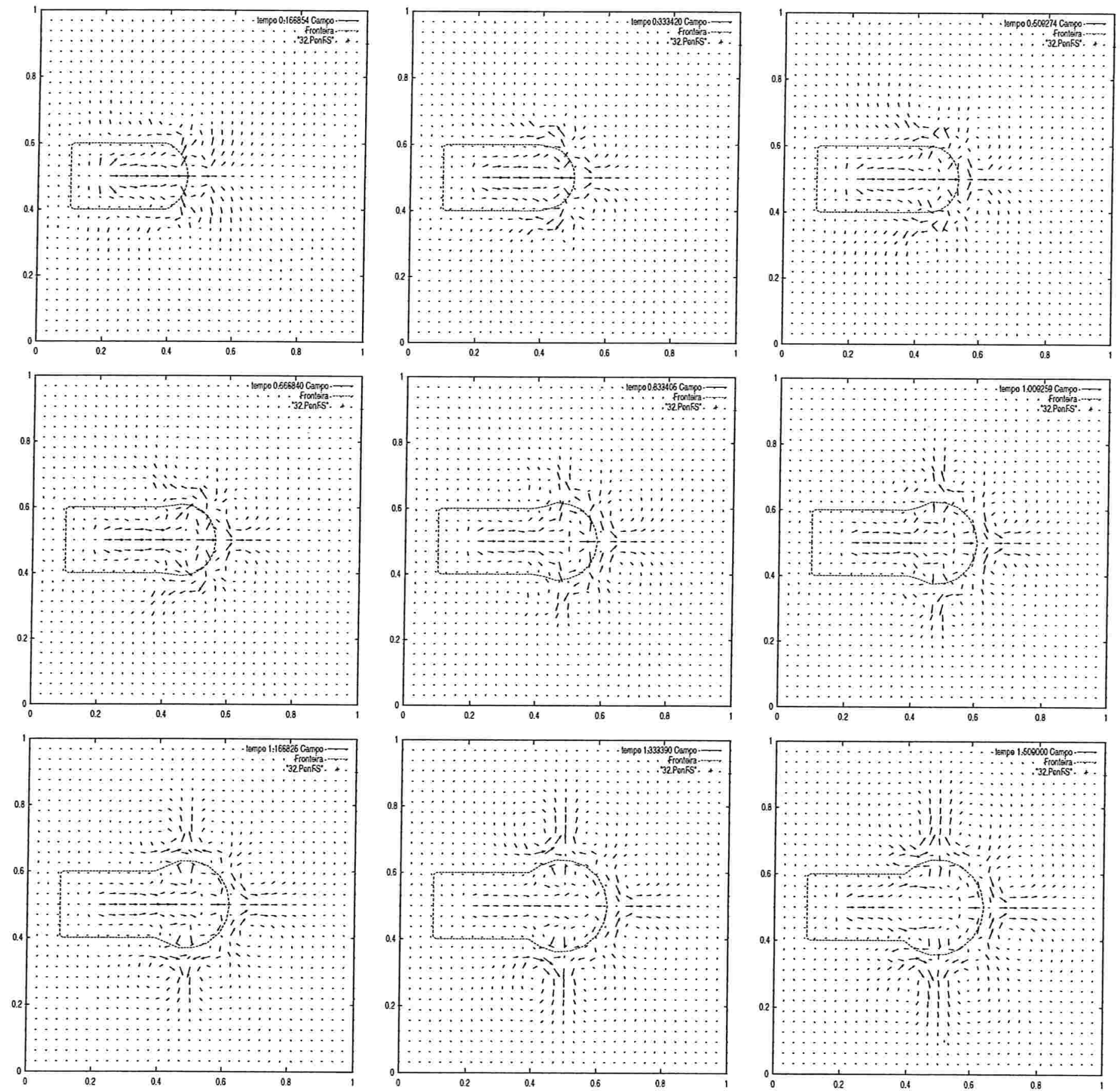

Figura 4.2: $R_{m}=10^{5} \frac{g}{\mathrm{~cm}^{3} . s}, \bar{P}_{m}=10^{3} \frac{\mathrm{g}}{\mathrm{cm} \cdot \mathrm{s}^{2}}, h_{o}=\frac{1}{32} \mathrm{e}_{\text {max }}=10^{6} \frac{d y n}{\mathrm{~cm}}$ 
Uma diferença adimensional superior a $10^{5}$ entre a resistência e a pressão associadas a cada fonte e sorvedouro, com $R_{m}>\bar{P}_{m}$, "retém" a expansão da fronteira dependendo dos valores atribuídos à pressão. Isto ocorre geralmente para

$$
\bar{P}_{m} \geq 10
$$

As Figuras 4.3 e 4.4 evidenciam essa situação. 

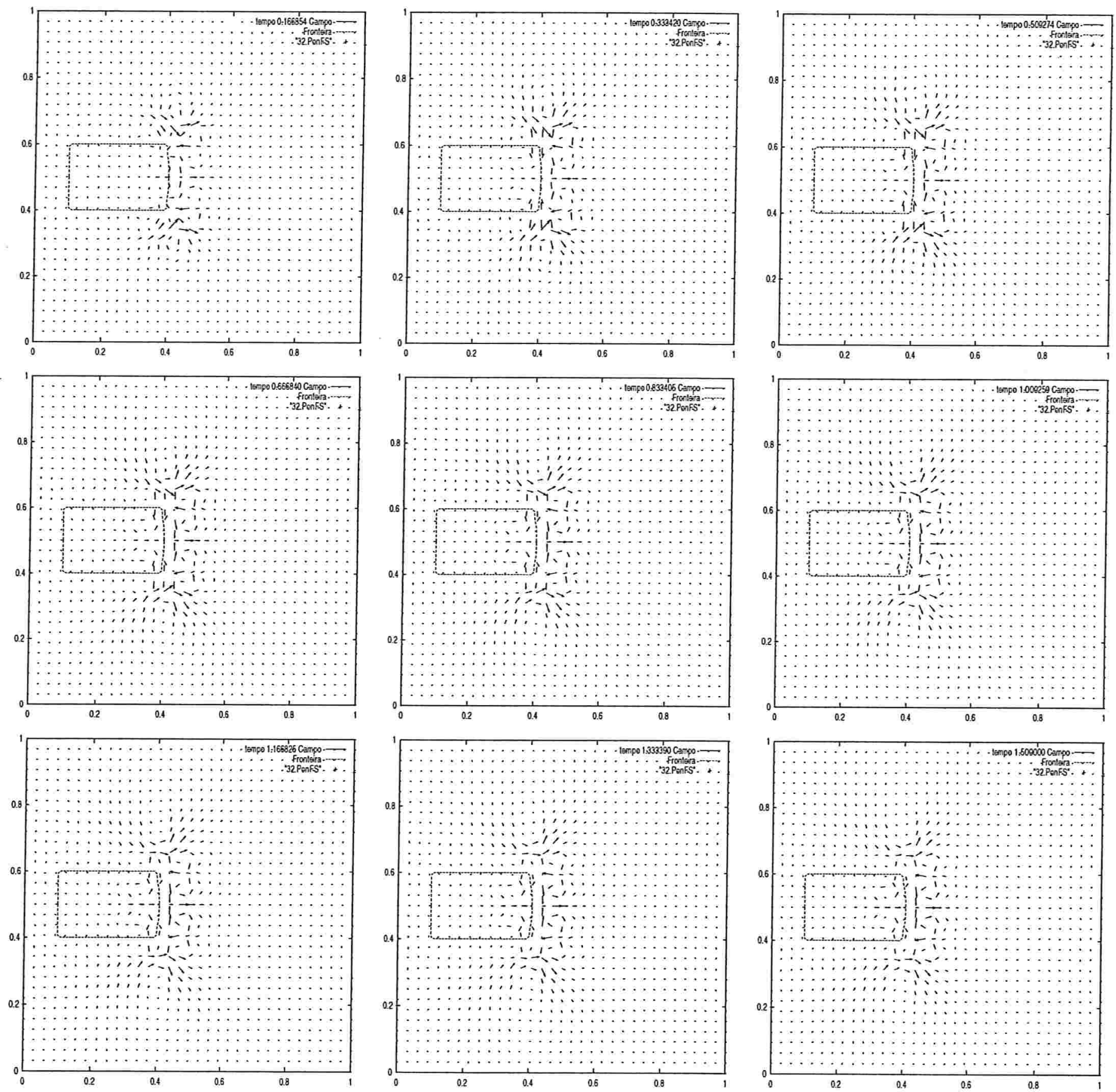

Figura 4.3: $R_{m}=10^{7} \frac{\mathrm{g}}{\mathrm{cm}^{3} \cdot \mathrm{s}}, \bar{P}_{m}=10^{3} \frac{\mathrm{g}}{\mathrm{cm} \cdot \mathrm{s}^{2}}, h_{o}=\frac{1}{32}$ e $\mathrm{k}_{\text {max }}=10^{6} \frac{\mathrm{dyn}}{\mathrm{cm}}$ 

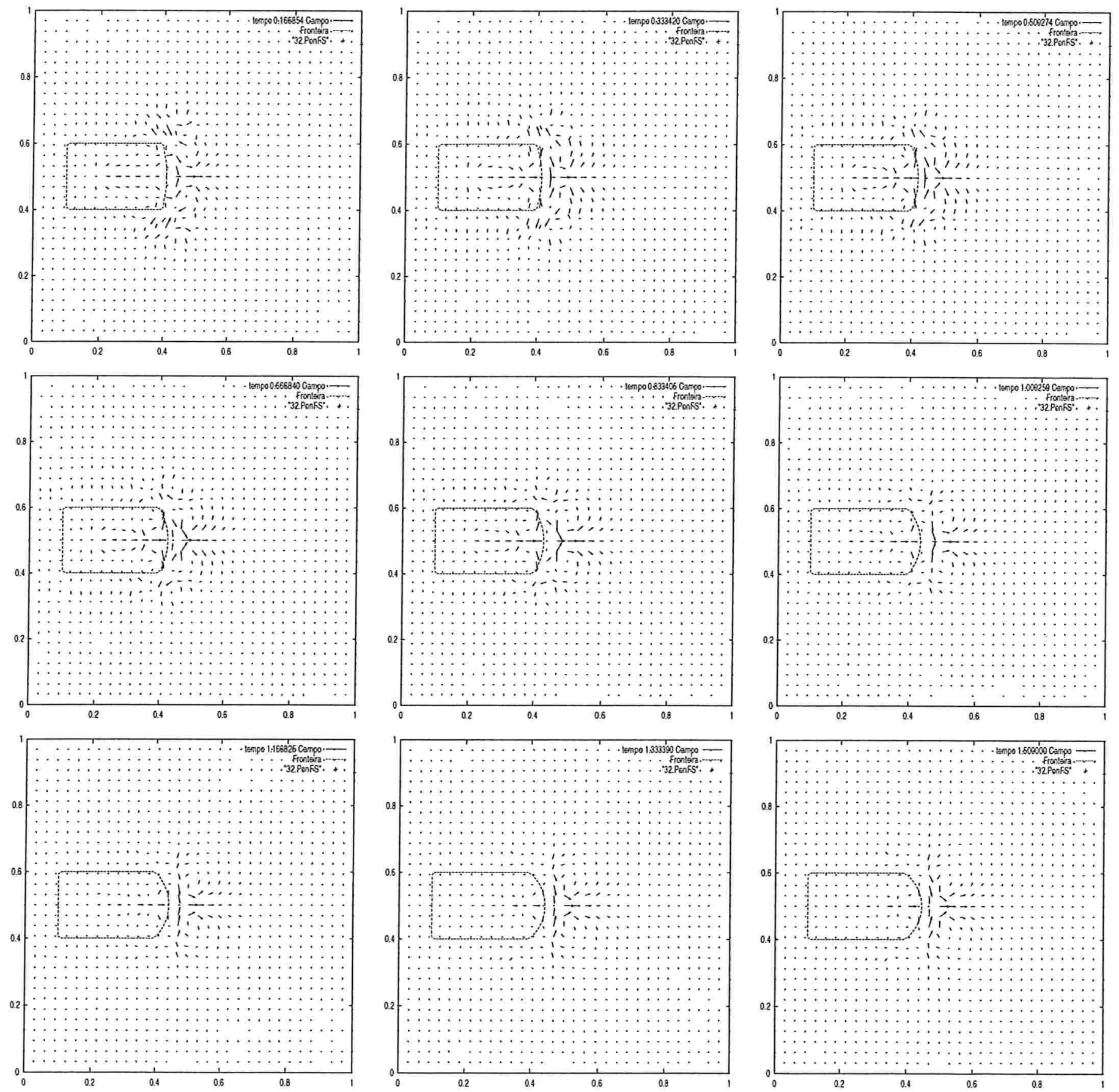

Figura 4.4: $R_{m}=10^{6} \frac{g}{\mathrm{~cm}^{3} \cdot s}, \bar{P}_{m}=10^{3} \frac{g}{\mathrm{~cm} \cdot \mathrm{s}^{2}}, h_{o}=\frac{1}{32} \mathrm{e} \mathrm{k}_{\max }=10^{6} \frac{\mathrm{dyn}}{\mathrm{cm}}$ 
Pode-se obter uma grande variação de área já no primeiro quadro da simulação $(t \approx$ $0.17 \mathrm{~s}$ ), o que é particularmente interessante à modelagem da expansão do alvéolo. As Figuras 4.5 e 4.6 ilustram testes nos quais uma diferença adimensional inferior a $10^{5}$ entre os valores da resistência e da pressão associadas a cada fonte e sorvedouro, com $R_{m}>\bar{P}_{m}$, produz essa variação. A assimetria verificada na simulação ilustrada pela Figura 4.6 se deve aos valores atribuídos à resistência, à pressão e ao tamanho do suporte. 

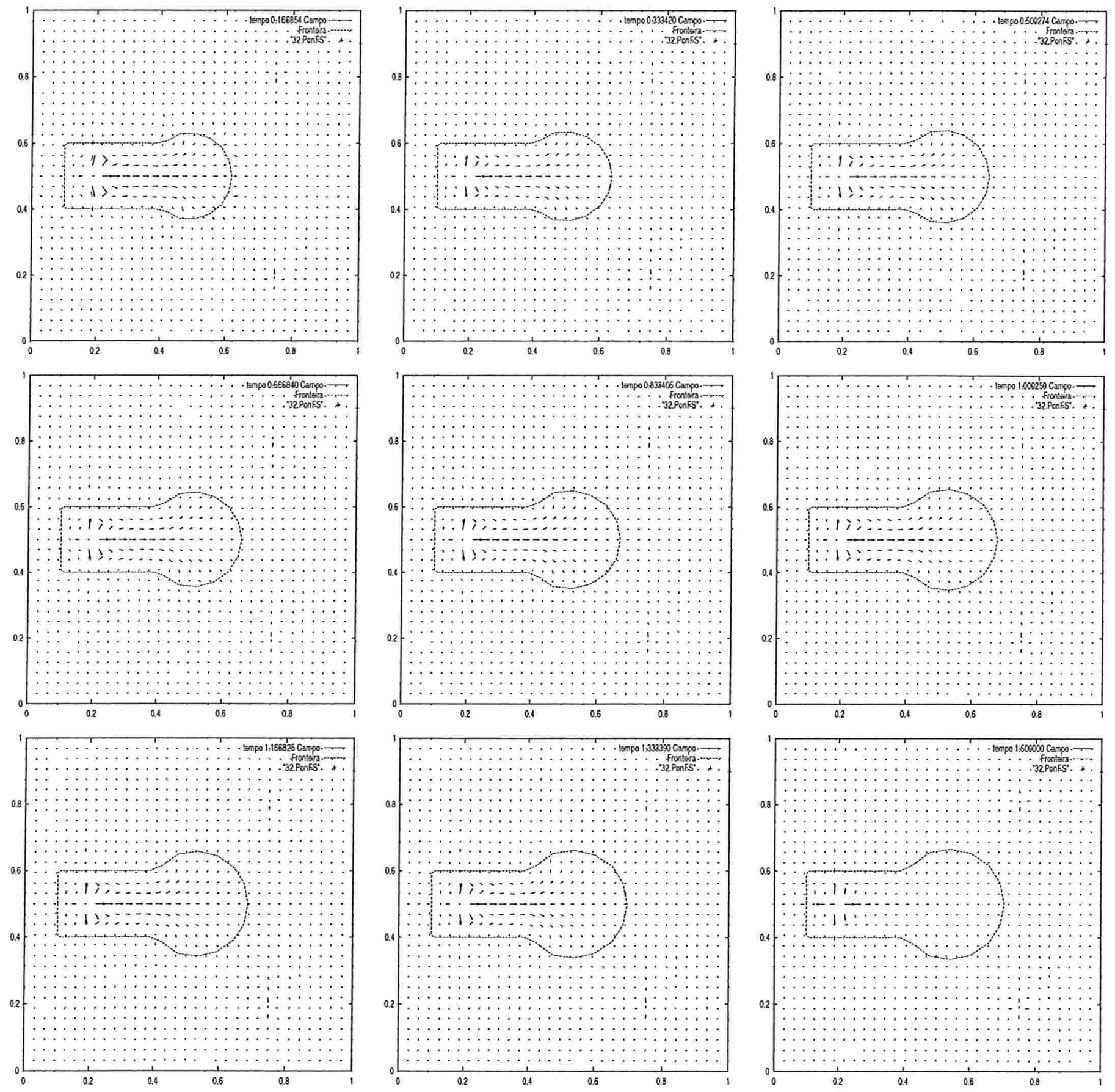

Figura 4.5: $R_{m}=10^{4} \frac{\mathrm{g}}{\mathrm{cm}^{3} \cdot \mathrm{s}}, \bar{P}_{m}=10^{2} \frac{\mathrm{g}}{\mathrm{cm} \cdot \mathrm{s}^{2}}, h_{o}=\frac{1}{64}$ e $\mathrm{k}_{\max }=10^{6} \frac{\mathrm{dyn}}{\mathrm{cm}}$ 

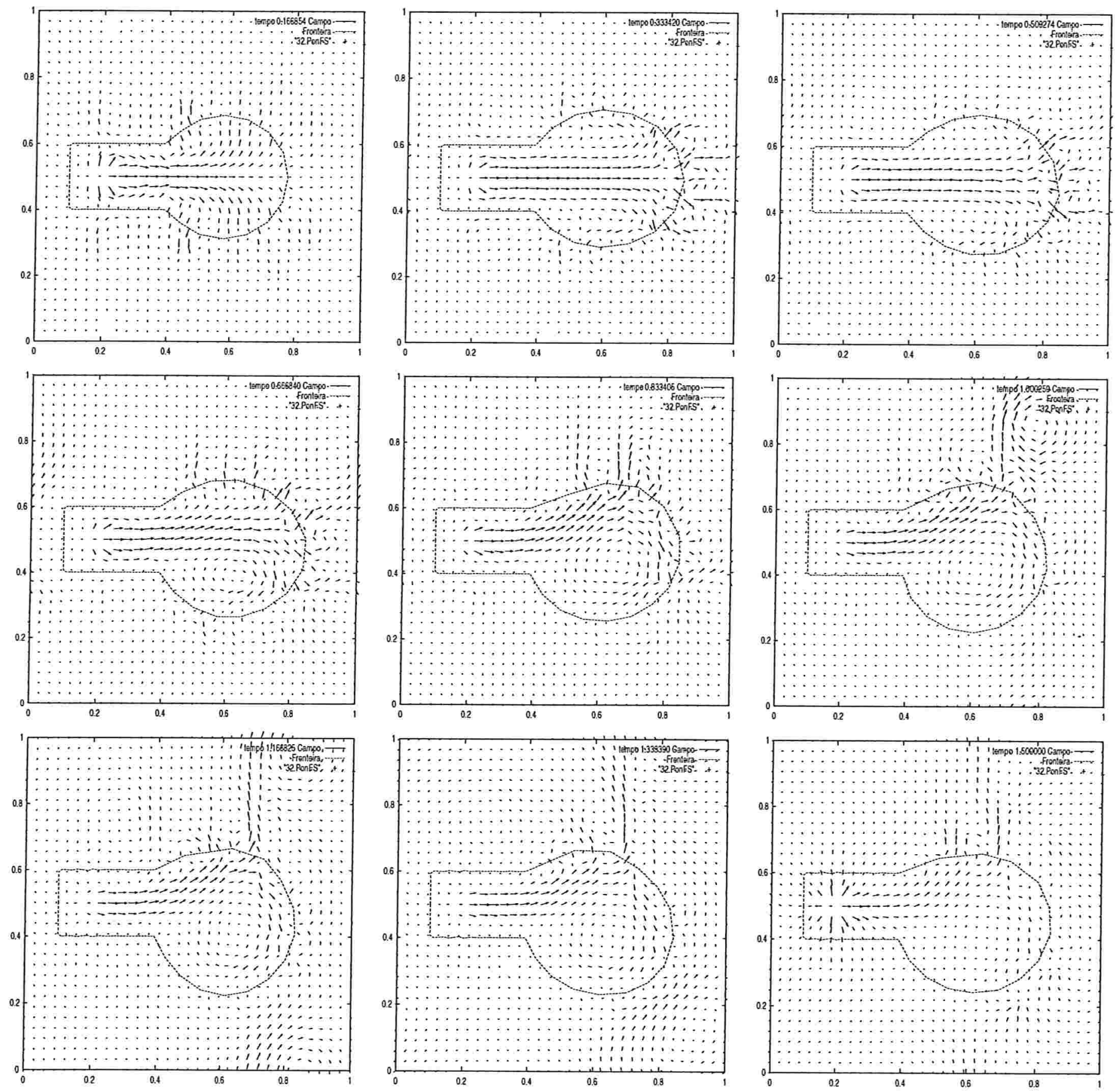

Figura 4.6: $R_{m}=10^{4} \frac{\mathrm{g}}{\mathrm{cm}^{3} \cdot s}, \bar{P}_{m}=10^{3} \frac{\mathrm{g}}{\mathrm{cm} \cdot \mathrm{s}^{2}}, h_{o}=\frac{1}{32} \mathrm{e}_{\max }=10^{6} \frac{\mathrm{dyn}}{\mathrm{cm}}$ 
A adequação dos valores da pressão, da resistência e do tamanho do suporte determina uma matriz $A$ estritamente diagonal dominante no sistema $A Q^{n+1}=B^{n+1}$. E simulações com expansões homogêneas da fronteira estão associadas a uma matriz $A$ estritamente diagonal dominante no cálculo da vazão $Q$ em todos os testes efetuados. Neles, uma diferença adimensional de aproximadamente $10^{5}$ entre a resistência $R_{m}$ e a pressão $\bar{P}_{m}$, $\operatorname{com} R_{m}>\bar{P}_{m}$ e $10^{2} \leq \bar{P}_{m} \leq 10^{4}$, garantiu a dominância diagonal da matriz $A$. Como contra-exemplo, menciona-se o teste efetuado para $R_{m}=10^{3} \frac{\mathrm{g}}{\mathrm{cm}^{3} . \mathrm{s}}, \bar{P}_{m}=10^{2} \frac{\mathrm{g}}{\mathrm{cm}_{\mathrm{s}} \mathrm{s}^{2}} \mathrm{e}$ $h_{o}=\frac{1}{32}$. A matriz $A$ neste caso não é diagonal dominante e a fronteira imersa é varrida para fora do domínio computacional já no primeiro quadro da simulação.

O que se observa para $k=5$ não necessariamente se repete para outros valores de $k$ ou quando o centro das fontes e sorvedouros é modificado. Contudo, todos os testes efetuados com $k=2,3,4,5,6,7,8,9,14$ ou 18 e $h_{o}=16,32$ ou 64 apresentaram resultados satisfatórios ao se empregar $R_{m}=10^{5} \frac{\mathrm{g}}{\mathrm{cm}^{3} . \mathrm{s}}$ e $\bar{P}_{m}=10^{3} \frac{\mathrm{g}}{\mathrm{cm} . \mathrm{s}^{2}}$. Vale ressaltar que alterações nos valores dos parâmetros $k, R_{m}, \bar{P}_{m}$ e $h_{o}$ implicam em se refazer os testes para observar se a expansão da fronteira imersa ocorre como desejado. Para exemplificar, usando-se $R_{m}=10^{4} \frac{\mathrm{g}}{\mathrm{cm}^{3} . \mathrm{s}}, \bar{P}_{m}=10^{2} \frac{\mathrm{g}}{\mathrm{cm} \cdot \mathrm{s}^{2}}$ e $h_{o}=\frac{1}{64}$, verifica-se que a fronteira é varrida para fora do domínio computacional já no primeiro quadro da simulação quando $k=9$, diferentemente do que acontece quando $k=5$ - Figura 4.5.

\subsection{Fronteira elástica}

Na simulação da expansão da superfície alveolar durante a inspiração, adotou-se uma geometria inicial pouco complexa - Figura 2.2 - que deve apresentar variação de área e, conseqüentemente, de formato no decorrer do tempo. A variação de área é controlada com base nas estimativas para a variação volumétrica do alvéolo na inspiração após $t=1.0 \mathrm{~s}$ e $t=1.5 s$ fornecidas pelas expressões (2.1) e (2.2).

A elasticidade da fronteira imersa é modelada da seguinte forma:

1o) emprego de uma função restauradora - equação (2.30) - para os pontos que simulam o bronquíolo respiratório. A rigidez dessa parte da fronteira é obtida pelo uso de uma constante de elasticidade elevada - $\mathrm{k}=10^{6} \frac{\mathrm{dyn}}{\mathrm{cm}}$;

$2^{\circ}$ ) emprego de uma força proveniente da generalização da lei de Hooke - equação (4.4) para os pontos que simulam o alvéolo. A elasticidade dessa parte da fronteira é provida 
pela utilização de valores distintos para a "constante" elástica $\mathrm{k}_{\text {elast }}=T_{0}$ em cada ponto e a evolução temporal desses valores.

A variação temporal da elasticidade associada aos pontos que simulam a membrana alveolar é obtida através de uma função $k^{*}[i]$ que depende da elasticidade $k[i]$ associada a cada ponto na configuração inicial $(t=0 s)$ adotada para a fronteira e do passo temporal $\Delta t$. Por exemplo, a função

$$
k^{*}[i]=e^{k[i] n \Delta t}
$$

associa a cada ponto uma elasticidade que varia com $n=1,2,3,4,5, \ldots, w$, onde

$$
w=\operatorname{int}\left(\frac{t_{\text {final }}}{\Delta t}\right)=\operatorname{int}\left(\frac{1.5 s}{\Delta t}\right)
$$

Na geometria adotada para a fronteira imersa, os extremos do segmento $\overline{B D}$, cuja expansão simula o alvéolo, têm coordenadas $(0.4,0.4)$ e $(0.4,0.6)$. A elasticidade $k[i]$ associada aos pontos $P$ do segmento é dada por

$$
\begin{array}{ll}
k[i]=X(i) & \text { se } P \in \overline{B E} \\
k[i]=1-X(i) & \text { se } P \in \overline{D E}
\end{array}
$$

onde $E$ é o ponto médio do segmento $\overline{B D}$ e $X(i)$ é a ordenada do ponto $P$. Modela-se desta maneira uma expansão simétrica dos segmentos $\overline{B E}$ e $\overline{D E}$.

Se a função $k^{*}[i]=e^{k[i] n \Delta t}$ for empregada para a variação temporal, então

$$
1<k^{*}[i] \leq e^{(0.6)(1.5)}
$$

Para determinar a área da região interior à fronteira imersa após $t=1.0 \mathrm{~s}$ e $t=1.5 \mathrm{~s}$ empregou-se a formulação integral para o cálculo da área de polígonos descrita e utilizada por Remigio [29].

A Tabela 4.1 lista as funções $k^{*}[i]$ testadas para a variação temporal dos valores da elasticidade $k[i]$, bem como a variação de área obtida após 1 e 1.5 segundo. A maior parte das funções escolhidas atribui valores mais baixos à elasticidade nos instantes de tempo iniciais, e valores mais elevados nos instantes de tempo finais. Essa escolha possibilita geralmente uma maior expansão da parte flexível da fronteira imersa nos dois terços iniciais $(0<t \leq 1.0 \mathrm{~s})$ do tempo total de simulação $(0<t \leq 1.5 \mathrm{~s})$. Em todos os casos analisados empregou-se $R_{m}=10^{5} \frac{\mathrm{g}}{\mathrm{cm}^{3} . \mathrm{s}}, \bar{P}_{m}=10^{3} \frac{\mathrm{g}}{\mathrm{cm} \cdot \mathrm{s}^{2}}, h_{o}=\frac{1}{32}, \rho=1 \frac{\mathrm{g}}{\mathrm{cm}^{3}}, \mu=10^{-2} \frac{\mathrm{g}}{\mathrm{cm} \cdot \mathrm{s}} \mathrm{e}$ 
$k=5$, sendo $k$ o número de fontes e sorvedouros introduzidos no domínio computacional. As cinco fontes têm centro no ponto $(0.2,0.5)$ e os sorvedouros estão centrados nos pontos $(0.75,0.2),(0.85,0.3),(0.9,0.5),(0.85,0.7)$ e $(0.75,0.8)$. Na primeira linha da Tabela 4.1 consta a variação de área obtida com $T_{o}=0$ para todos os pontos do segmento $\overline{B D}$ no decorrer do tempo. Esse valor para a elasticidade fornece a variação de área máxima para os parâmetros adotados, isto porque a fronteira imersa não exerce força alguma e os pontos que a representam são então simplesmente carregados pelo fluxo.

\begin{tabular}{|c|c|c|c|c|c|}
\hline № & $\mathbf{k}[\mathrm{i}]-\overline{\mathrm{BE}}$ & $\mathrm{k}[\mathrm{i}]-\overline{\mathrm{DE}}$ & $\mathbf{k}^{*}[\mathbf{i}]$ & $\Delta_{\mathrm{AREA}}(\mathrm{t}=1.0 \mathrm{~s})$ & $\Delta_{\mathrm{AREA}}(\mathrm{t}=1.5 \mathrm{~s})$ \\
\hline 01 & 0.0 & 0.0 & 0.0 & $84.32 \%$ & $125.58 \%$ \\
\hline 02 & $X(i)$ & $1-X(i)$ & $\mathrm{k}[\mathrm{i}] \mathrm{n} \Delta \mathrm{t}$ & $78.9 \%$ & $106.18 \%$ \\
\hline 03 & $X(i)$ & $1-X(i)$ & $e^{k[i] n \Delta t}$ & $68.33 \%$ & $82.88 \%$ \\
\hline 04 & $X(i)$ & $1-X(i)$ & $k[i] e^{n \Delta t}$ & $71.69 \%$ & $85.6 \%$ \\
\hline 05 & $X(i)$ & $1-X(i)$ & $n \Delta t e^{k[i]}$ & $71.56 \%$ & $81.97 \%$ \\
\hline 06 & $X(i)$ & $1-X(i)$ & $\operatorname{sen}(k[i] n \Delta t)$ & $78.99 \%$ & $107.2 \%$ \\
\hline 07 & $X(i)$ & $1-X(i)$ & $(\mathrm{k}[\mathrm{i}] \mathrm{n} \Delta \mathrm{t})^{2}$ & $81.89 \%$ & $112.97 \%$ \\
\hline 08 & $X(i)$ & $1-X(i)$ & $(k[i] n \Delta t-2)^{2}$ & $56.84 \%$ & $79.14 \%$ \\
\hline 09 & $X(i)$ & $1-X(i)$ & $e^{\operatorname{sen}(k[i] n \Delta t)}$ & $68.43 \%$ & $83.65 \%$ \\
\hline 10 & $X(i)$ & $1-X(i)$ & $\operatorname{sen}\left(e^{k[i] n \Delta t}\right)$ & $72.02 \%$ & $96.66 \%$ \\
\hline 11 & $X(i)$ & $1-X(i)$ & $\operatorname{sen}(k[i] n \Delta t)+e^{k[i] n \Delta t}$ & $65.64 \%$ & $76.85 \%$ \\
\hline 12 & $X(i)$ & $1-X(i)$ & $\left(e^{k[i] n \Delta t}\right)^{\operatorname{sen}(k[i] n \Delta t)}$ & $70.64 \%$ & $88.77 \%$ \\
\hline 13 & $X(i)$ & $1-X(i)$ & $(\operatorname{sen}(k[i] n \Delta t))^{e^{k[i] n \Delta t}}$ & $80.51 \%$ & $112.63 \%$ \\
\hline 14 & $X(i)$ & $1-X(i)$ & $\operatorname{tg}(k[i] n \Delta t)$ & $78.71 \%$ & $103.8 \%$ \\
\hline
\end{tabular}

Tabela 4.1: Funções para a variação temporal da elasticidade $-R_{m}=10^{5} \frac{\mathrm{g}}{\mathrm{cm}^{3} . \mathrm{s}}, \bar{P}_{m}=10^{3} \frac{\mathrm{g}}{\mathrm{cm} \cdot \mathrm{s}^{2}}$, $h_{o}=\frac{1}{32}, \rho=1 \frac{g}{\mathrm{~cm}^{3}}, \mu=10^{-2} \frac{g}{\mathrm{~cm} . s}, k=5, N=32$

Para cinco fontes e cinco sorvedouros e as funções presentes na Tabela 4.1 não se obteve a variação de área fornecida pelas expressões (2.1) e (2.2). Porém, a diferença entre as variações após $t=1.0 \mathrm{~s}$ e $t=1.5 \mathrm{~s}$ - cerca de $30 \%$ nos melhores casos - é próxima àquela fornecida pelas mesmas expressões - cerca de 33.5\%. As funções destacadas na Tabela 4.1 são aquelas que proporcionaram uma variação de área mais próxima à estimada.

Quanto à função

$$
k^{*}[i]=e^{k[i] n \Delta t}
$$


primeira função estruturada para a evolução temporal da elasticidade, a variação de área obtida após $1.5 \mathrm{~s}$ fica bem aquém do valor desejado. Então, testou-se uma função da forma

$$
k^{*}[i]=e^{\alpha k[i] n \Delta t-\beta}
$$

Para $\alpha=1$ e $\beta>0$, a função atribui valores menores à elasticidade, o que provoca maior expansão da fronteira. Exemplificando, quando $\beta=1$, a variação de área verificada após $t=1.0 \mathrm{~s}$ e $t=1.5 \mathrm{~s}$ é de, respectivamente, $76.62 \%$ e $103.41 \%$, valores mais próximos dos obtidos com as funções em destaque na Tabela 4.1.

Considerando-se $\alpha=3.6$ e $\beta=0$, a função atribui à elasticidade no instante final $t=$ $1.5 \mathrm{~s}$ valores mais condizentes com a tensão superficial existente na camada de surfactante que reveste internamente os alvéolos pulmonares, determinando uma variação de área de $49.94 \%$ após $t=1.0 \mathrm{~s}$ e de $38.65 \%$ após $t=1.5 \mathrm{~s}$. Assim, o emprego dessa função gera redução de área no intervalo de tempo $1.0<t \leq 1.5$. Isto ocorre devido ao aumento brusco do valor da elasticidade em relação ao intervalo de tempo inicial $0<t \leq 1.0$. Poder-se-ia então empregar essa função ou outra similar para modelar o recuo da parede alveolar verificado no movimento de expiração.

É importante salientar que, além dos valores atribuídos à elasticidade no decorrer do tempo, a posição dos pontos $X(s-1)$ e $X(s+1)$ aos quais o ponto $X(s)$ está "amarrado" - equação (3.46) - também é determinante no cálculo da força exercida por este último ponto. A mesma equação deixa claro que se $\Delta s$ for muito pequeno, o que ocorre quando se emprega uma malha computacional mais fina, a força exercida pelos pontos da fronteira imersa será maior. Assim, a mesma função pode se comportar de maneira completamente distinta em malhas computacionais com passo espacial $h$ diferente no que diz respeito à variação de área.

Como o fluxo de ar está associado ao número de fontes e sorvedouros inseridos no modelo, analisa-se agora o comportamento das mesmas funções mediante a introdução de um número maior de fontes e sorvedouros.

\subsubsection{Ajuste do número $k$ de fontes e sorvedouros}

A Tabela 4.2 relaciona a variação de área obtida com a introdução de sete fontes e sete sorvedouros no domínio computacional. As fontes têm centro no ponto $(0.2,0.5)$ e os 
sorvedouros estão centrados em $(0.65,0.15),(0.75,0.2),(0.85,0.3),(0.9,0.5),(0.85,0.7)$, $(0.75,0.8)$ e $(0.65,0.85)$.

\begin{tabular}{|c|c|c|c|c|c|}
\hline № & $\mathrm{k}[\mathrm{i}]-\overline{\mathrm{BE}}$ & $\mathrm{k}[\mathrm{i}]-\overline{\mathrm{DE}}$ & $\mathrm{k}^{*}[\mathrm{i}]$ & $\Delta_{\mathrm{AREA}}(\mathrm{t}=1.0 \mathrm{~s})$ & $\Delta_{\mathrm{AREA}}(\mathrm{t}=1.5 \mathrm{~s})$ \\
\hline 01 & 0.0 & 0.0 & 0.0 & $119.18 \%$ & $176.65 \%$ \\
\hline 02 & $X(i)$ & $1-X(i)$ & $\mathrm{k}[\mathrm{i}] \mathrm{n} \Delta \mathrm{t}$ & $109.85 \%$ & $144.78 \%$ \\
\hline 03 & $X(i)$ & $1-X(i)$ & $e^{k[i] n \Delta t}$ & $92.82 \%$ & $103.43 \%$ \\
\hline 04 & $X(i)$ & $1-X(i)$ & $e^{(3.6) k[i] n \Delta t}$ & $71.95 \%$ & $55.25 \%$ \\
\hline 05 & $X(i)$ & $1-X(i)$ & $k[i] e^{n \Delta t}$ & $97.97 \%$ & $108.52 \%$ \\
\hline 06 & $X(i)$ & $1-X(i)$ & $n \Delta t e^{k[i]}$ & $96.58 \%$ & $102.11 \%$ \\
\hline 07 & $X(i)$ & $1-X(i)$ & $\operatorname{sen}(k[i] n \Delta t)$ & $110 \%$ & $146.16 \%$ \\
\hline 08 & $X(i)$ & $1-X(i)$ & $(k[i] n \Delta t)^{2}$ & $114.91 \%$ & $156.28 \%$ \\
\hline 09 & $X(i)$ & $1-X(i)$ & $(k[i] n \Delta t-2)^{2}$ & $79.81 \%$ & $101.06 \%$ \\
\hline 10 & $X(i)$ & $1-X(i)$ & $e^{\operatorname{sen}(k[i] n \Delta t)}$ & $93.01 \%$ & $105.09 \%$ \\
\hline 11 & $X(i)$ & $1-X(i)$ & $\operatorname{sen}\left(e^{k[i] n \Delta t}\right)$ & $99.32 \%$ & $127.76 \%$ \\
\hline 12 & $X(i)$ & $1-X(i)$ & $\operatorname{sen}(k[i] n \Delta t)+e^{k[i] n \Delta t}$ & $88.71 \%$ & $93.61 \%$ \\
\hline 13 & $X(i)$ & $1-X(i)$ & $\left.\left(e^{k[i] n \Delta t}\right)^{\operatorname{sen}(k[i] n \Delta t}\right)$ & $96.71 \%$ & $114.52 \%$ \\
\hline 14 & $X(i)$ & $1-X(i)$ & $(\operatorname{sen}(k[i] n \Delta t))^{e^{k[i] n \Delta t}}$ & $112.47 \%$ & $155.65 \%$ \\
\hline 15 & $X(i)$ & $1-X(i)$ & $\operatorname{tg}(k[\mathrm{i}] \mathrm{n} \Delta \mathrm{t})$ & $109.53 \%$ & $141.56 \%$ \\
\hline
\end{tabular}

Tabela 4.2: Funções para a variação temporal da elasticidade $-R_{m}=10^{5} \frac{g}{\mathrm{~cm}^{3} . s}, \bar{P}_{m}=10^{3} \frac{\mathrm{g}}{\mathrm{cm} \cdot \mathrm{s}^{2}}$, $h_{o}=\frac{1}{32}, \rho=1 \frac{\mathrm{g}}{\mathrm{cm}^{3}}, \mu=10^{-2} \frac{\mathrm{g}}{\mathrm{cm} \cdot \mathrm{s}}, k=7, N=32$

A utilização de sete fontes e sete sorvedouros, assim como ocorreu para cinco fontes e cinco sorvedouros, não produz a variação de área desejada nos dois intervalos de tempo para nenhuma das funções testadas. As funções destacadas na Tabela 4.2 são aquelas que melhor aproximam a variação de área àquela estimada nas expressões (2.1) e (2.2). Apesar da variação insuficiente para $t=1.0 \mathrm{~s}, \mathrm{com}$ as funções $(k[i] n \Delta t)^{2} \mathrm{e}(\operatorname{sen}(k[i] n \Delta t))^{e^{k[i] n \Delta t}}$ obtém-se uma variação de área próxima à desejada para $t=1.5 \mathrm{~s}$.

Quanto à função da forma $k^{*}[i]=e^{\alpha k[i] n \Delta t-\beta}, \alpha=1 \mathrm{e} \beta=1.5$ induzem após $t=1.0 \mathrm{~s}$ e $t=1.5 \mathrm{~s}$ variações na área de, respectivamente, $110.17 \%$ e $152.1 \%$, valores mais próximos dos esperados do que aqueles determinados pela função $k^{*}[i]=e^{k[i] n \Delta t}$.

A função $k^{*}[i]=(k[i] n \Delta t-2)^{2}$ produz resultados bem aquém dos requeridos. Porém, 
considerando-se uma função da forma

$$
k^{*}[i]=(k[i] n \Delta t-\gamma)^{2}
$$

verifica-se que $\gamma=1$ determina uma variação de área de $107.12 \%$ e $153.99 \%$ após $t=1.0 \mathrm{~s}$ e $t=1.5 \mathrm{~s}$, respectivamente, valores consideravelmente mais próximos dos estimados do que aqueles obtidos com a função $k^{*}[i]=(k[i] n \Delta t-2)^{2}$.

$\mathrm{Na}$ busca de variações realmente próximas dos valores estimados em (2.1) e (2.2), testase agora as funções para a variação temporal da elasticidade mediante a inserção de nove fontes e nove sorvedouros no domínio computacional. A Tabela 4.3 relaciona a variação de área obtida com $k=9$. As fontes têm centro no ponto $(0.2,0.5)$ e os sorvedouros estão centrados em $(0.55,0.1),(0.65,0.15),(0.75,0.2),(0.85,0.3),(0.9,0.5),(0.85,0.7)$, $(0.75,0.8) ;(0.65,0.85)$ e $(0.55,0.9)$.

\begin{tabular}{|l|l|l|l|l|l|}
\hline $\mathbf{N o}$ & $\mathbf{k}[\mathbf{i}]-\overline{\mathbf{B E}}$ & $\mathbf{k}[\mathbf{i}]-\overline{\mathbf{D E}}$ & $\mathbf{k}^{*}[\mathbf{i}]$ & $\Delta_{\text {AREA }}(\mathbf{t}=1.0 \mathbf{s})$ & $\Delta_{\text {AREA }}(\mathbf{t}=1.5 \mathbf{s})$ \\
\hline 01 & 0.0 & 0.0 & 0.0 & $153.77 \%$ & $225.9 \%$ \\
\hline 02 & $X(i)$ & $1-X(i)$ & $k[i] n \Delta t$ & $142.4 \%$ & $174.56 \%$ \\
\hline 03 & $X(i)$ & $1-X(i)$ & $e^{k[i] n \Delta t}$ & $114.99 \%$ & $130.32 \%$ \\
\hline 04 & $X(i)$ & $1-X(i)$ & $e^{(3.6) k[i] n \Delta t}$ & $84.36 \%$ & $60.18 \%$ \\
\hline 05 & $X(i)$ & $1-X(i)$ & $k[i] e^{n \Delta t}$ & $123.68 \%$ & $134.79 \%$ \\
\hline 06 & $X(i)$ & $1-X(i)$ & $n \Delta t e^{k[i]}$ & $121.29 \%$ & $128.97 \%$ \\
\hline 07 & $X(i)$ & $1-X(i)$ & $\operatorname{sen}(k[i] n \Delta t)$ & $142.69 \%$ & $176.51 \%$ \\
\hline 08 & $X(i)$ & $1-X(i)$ & $(k[i] n \Delta t)^{2}$ & $148.29 \%$ & $185.52 \%$ \\
\hline 09 & $X(i)$ & $1-X(i)$ & $(k[i] n \Delta t-2)^{2}$ & $97.48 \%$ & $121.5 \%$ \\
\hline 10 & $X(i)$ & $1-X(i)$ & $e^{\operatorname{sen}(k[i] n \Delta t)}$ & $115.3 \%$ & $131.8 \%$ \\
\hline 11 & $X(i)$ & $1-X(i)$ & $\operatorname{sen}\left(\mathrm{e}^{\mathbf{k}[\mathbf{i}] \mathbf{n} \Delta t}\right)$ & $125.41 \%$ & $156.34 \%$ \\
\hline 12 & $X(i)$ & $1-X(i)$ & $\operatorname{sen}(k[i] n \Delta t)+e^{k[i] n \Delta t}$ & $107.97 \%$ & $115.86 \%$ \\
\hline 13 & $X(i)$ & $1-X(i)$ & $\left(e^{k[i] n \Delta t}\right) \operatorname{sen}(k[i] n \Delta t)$ & $121.51 \%$ & $141.24 \%$ \\
\hline 14 & $X(i)$ & $1-X(i)$ & $(\operatorname{sen}(k[i] n \Delta t))^{e^{k i] n \Delta t}}$ & $145.29 \%$ & $187.68 \%$ \\
\hline 15 & $X(i)$ & $1-X(i)$ & $t g(k[i] n \Delta t)$ & $141.73 \%$ & $169.98 \%$ \\
\hline
\end{tabular}

Tabela 4.3: Funções para a variação temporal da elasticidade $-R_{m}=10^{5} \frac{\mathrm{g}}{\mathrm{cm}^{3} \cdot s}, \bar{P}_{m}=10^{3} \frac{\mathrm{g}}{\mathrm{cm} \cdot \mathrm{s}^{2}}$, $h_{o}=\frac{1}{32}, \rho=1 \frac{g}{\mathrm{~cm}^{3}}, \mu=10^{-2} \frac{\mathrm{g}}{\mathrm{cm} \cdot \mathrm{s}}, k=9, N=32$

O emprego de nove fontes e nove sorvedouros produz uma variação de área muito próxima àquelas indicadas pelas expressões (2.1) e (2.2) quando a função 
para a variação temporal da elasticidade

$$
k^{*}[i]=\operatorname{sen}\left(e^{k[i] n \Delta t}\right)
$$

é empregada. Os valores obtidos para a variação de área podem ainda ser adequados pelo reposicionamento dos sorvedouros.

Em relação à função da forma

$$
k^{*}[i]=e^{\alpha k[i] n \Delta t-\beta}
$$

$\alpha=1$ e $\beta=0.5$ determinam uma variação de área de $128.18 \%$ após $t=1.0$ s e de $152.42 \%$ após $t=1.5 \mathrm{~s}$. No que diz respeito à função

$$
k^{*}[i]=(k[i] n \Delta t-\gamma)^{2}
$$

$\gamma=1.4$ provoca uma variação de área de $122.9 \%$ após $t=1.0 \mathrm{~s}$ e de $167.19 \%$ após $t=1.5 \mathrm{~s}$. Nos dois casos, as variações verificadas estão próximas dos valores estimados para a expansão do alvéolo.

Em todos os testes realizados até o momento, empregou-se um fluido de densidade $\rho=1 \frac{\mathrm{g}}{\mathrm{cm}^{3}}$ e viscosidade $\mu=10^{-2} \frac{\mathrm{g}}{\mathrm{cm} . \mathrm{s}}$, o que equivale a utilizar um número de Reynolds de aproximadamente $4 \mathrm{com}$ as funções que induzem uma variação de área próxima àquela estimada para o alvéolo. Contudo, é sabido que esses valores são elevados quando o fluido em questão é o ar escoando pelas ramificações finais da árvore respiratória durante o movimento normal de inspiração (Apêndices A e B).

\subsection{Valores fisiológicos para a densidade $\rho$ e a visco- sidade $\mu$ do fluido}

A introdução de valores fisiológicos para a densidade e a viscosidade do fluido no programa computacional produziu sempre resultados instáveis em uma malha computacional $32 \times 32$.

Testou-se então em uma malha $128 \times 128$ algumas funções $k^{*}[i]$ para a evolução temporal 
da elasticidade. Empregou-se

$$
\begin{aligned}
& \rho=1.21 \times 10^{-3} \frac{\mathrm{g}}{\mathrm{cm}^{3}} \\
& \mu=1.9 \times 10^{-4} \frac{\mathrm{g}}{\mathrm{cm} . \mathrm{s}}
\end{aligned}
$$

valores mais próximos da densidade e da viscosidade do ar que escoa no aparelho respiratório do que aqueles empregados anteriormente (Apêndices A e B). Esses valores determinam um número de Reynolds de cerca de 0.3 quando se usa uma função que provoca uma variação de área condizente com a estimada nas expressões (2.1) e (2.2).

Um inconveniente do uso de malhas computacionais mais finas é o tempo de execução do programa, o qual totaliza aproximadamente $13 h$ para as funções testadas em uma máquina Sam Enterprise 3000, contrariamente aos cerca de 5 min necessários à execução do programa em uma malha $32 \times 32$ na mesma máquina quando se utiliza $\rho=1.0 \frac{\mathrm{g}}{\mathrm{cm}^{3}} \mathrm{e}$ $\mu=10^{-2} \frac{\mathrm{g}}{\mathrm{cm} \cdot \mathrm{s}}$.

A utilização de valores fisiológicos para $\rho$ e $\mu$ exige a adequação de outros parâmetros além do refinamento da malha, como a redução do suporte $h_{o}$, e altera significativamente a variação de área.

Empregando-se $k=9, R_{m}=10^{5} \frac{g}{\mathrm{~cm}^{3} \cdot s}, \bar{P}_{m}=10^{3} \frac{\mathrm{g}}{\mathrm{cm} \cdot \mathrm{s}^{2}}$ e $h_{o}=\frac{1}{64}$, a função

$$
k^{*}[i]=e^{k[i] n \Delta t}
$$

determina uma variação excessiva de área - $145.5 \%$ e $217.95 \%$ - nos instantes $t=1.0 \mathrm{~s}$ e $t=1.5 \mathrm{~s}$, o que não ocorre com valores não fisiológicos para a densidade e a viscosidade em uma malha mais grossa.

Algumas das demais funções testadas induziram uma variação de área adequada apenas para um dos dois instantes de tempo. Decidiu-se testar então uma função definida por sentenças distintas para cada um dos intervalos de tempo. Com a função

$$
k^{*}[i]=\left\{\begin{array}{l}
(k[i] n \Delta t-5)^{2}, \quad \text { se } 0<n \Delta t \leq 1.0 \\
(k[i] n \Delta t-7)^{2}, \quad \text { se } 1.0<n \Delta t \leq 1.5
\end{array}\right.
$$

obtém-se após $t=1.0 \mathrm{~s}$ e $t=1.5 \mathrm{~s}$ uma variação de área de $128.75 \%$ e $181.58 \%$, respectivamente. Adotando-se

$$
k^{*}[i]=(k[i] n \Delta t-10)^{2}
$$


para o segundo intervalo, verifica-se uma variação de área de $157.28 \%$. Encontram-se assim variações próximas às estimadas nas expressões (2.1) e (2.2).

Alterando-se o número $k$ de fontes e sorvedouros, constata-se novamente que algumas funções determinam variações de área adequadas apenas em um dos instantes de tempo. Com a reestruturação do algoritmo para modificar o número $k$ de fontes e sorvedouros ao longo do tempo de execução, poder-se-ia controlar a expansão do segmento $\overline{B D}$ nos intervalos de tempo $0<t \leq 1.0$ e $1.0<t \leq 1.5$. A redução do número de fontes e sorvedouros no segundo intervalo de tempo simularia uma condição fisiológica: uma variação de área menor em relação ao intervalo inicial devido a uma redução do fluxo de gás e, por conseguinte, da pressão.

\subsection{Um modelo para dois alvéolos}

Sabe-se que os alvéolos pulmonares estão agrupados em sacos. Assim, torna-se interessante simular a expansão de mais de um alvéolo simultaneamente. Pode-se então estender o modelo para um alvéolo ilustrado na Figura 3.7 para dois alvéolos.

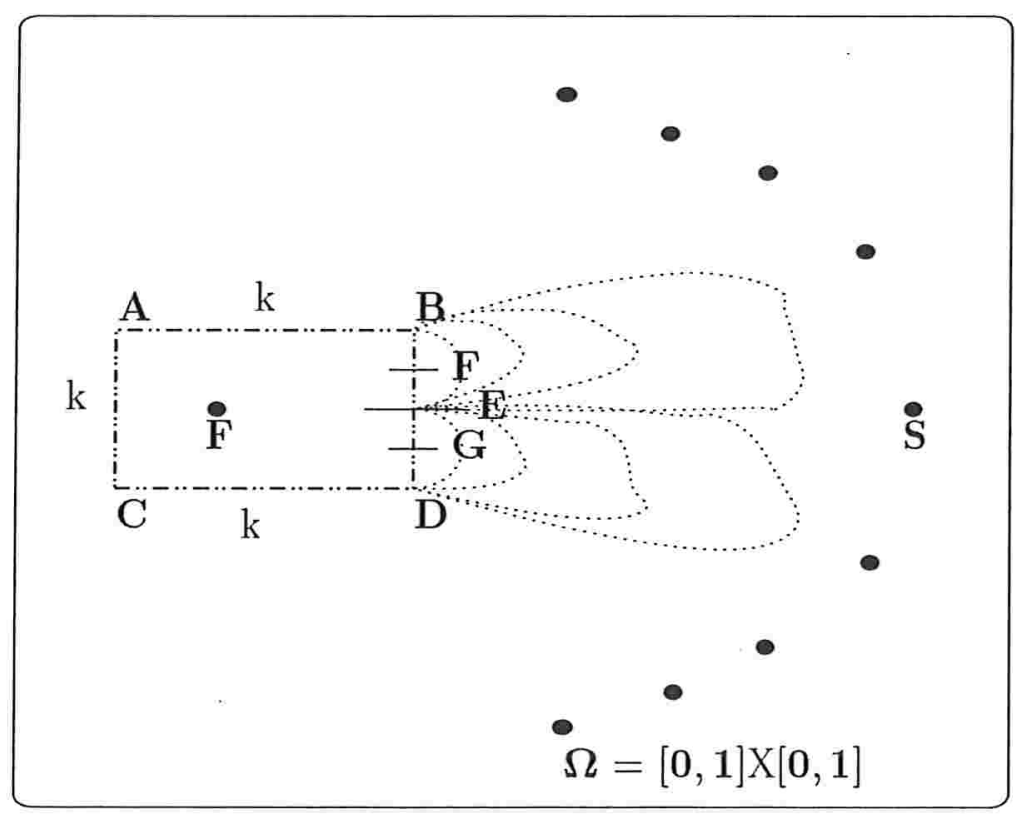

Figura 4.7: Modelo para dois alvéolos pulmonares

Na Figura $4.7, F(0.4,0.55)$ e $G(0.4,0.45)$ são os pontos médios dos segmentos $\overline{B E}$ e 
$\overline{D E}$, respectivamente, e os valores atribuídos à elasticidade dos pontos do segmento $\overline{B D}$ na configuração inicial $(t=0 s)$ da fronteira imersa são calculados da seguinte maneira:

- Ponto E: modelo de força restauradora com $\mathrm{k}_{\text {elast }}=\mathrm{k}=10^{6} \frac{\mathrm{dyn}}{\mathrm{cm}}$. Objetiva-se dessa forma manter o ponto E imóvel no decorrer do tempo;

- Segmento $\overline{\mathrm{BF}}$ :

$$
k[i]=X(i)
$$

sendo $X(i)$ a ordenada do ponto na configuração inicial da fronteira;

\section{- Segmento $\overline{\mathrm{EF}}$ :}

$$
k[i]=1.1-X(i)
$$

- Segmento $\overline{\mathrm{EG}}$ :

$$
k[i]=0.1+X(i)
$$

\section{- Segmento DG:}

$$
k[i]=1.0-X(i)
$$

As expressões (4.9) a (4.12) permitem uma expansão simétrica dos segmentos $\overline{B E}$ e $\overline{D E}$, desde que se use a mesma função para a variação temporal da elasticidade em ambos.

Como os alvéolos não são uniformemente ventilados, nem todos sofrem a mesma expansão assim como nem todos participam simultaneamente do processo respiratório. Em algumas doenças, como o enfisema e a fibrose, a complacência dos pulmões sofre alterações significativas. Em outros casos, como a existência de tumores ou edemas, podem ocorrer alterações locais na complacência. Adotando-se funções distintas para a variação temporal da elasticidade nos segmentos $\overline{B E}$ e $\overline{D E}$, pode-se simular duas expansões diferenciadas, imitando assim o que ocorre em pulmões sadios ou doentes.

O modelo pensado para simular um alvéolo pode ser generalizado para a simulação de $n$ alvéolos. Na Figura 4.7, aplicando-se o modelo de força restauradora com

$$
\mathrm{k}=10^{6} \frac{d y n}{\mathrm{~cm}}
$$


aos pontos $E, F$ e $G$, tem-se a expansão de quatro segmentos. Dividindo-se o segmento $\overline{B D}$ em $n$ partes, não necessariamente iguais, e aplicando-se o modelo de força restauradora aos $(n-1)$ pontos que dividem o segmento, pode-se simular a expansão de $n$ segmentos. Obviamente, torna-se necessário testar novos valores para os parâmetros que permitam expansões com variação de área condizente às esperadas para cada um dos alvéolos. 


\section{Simulações}

Compara-se neste capítulo as variações na área da região interna à fronteira imersa bem como na geometria desta quando se emprega um valor constante para a elasticidade ou se atribui a ela valores variáveis no tempo. As simulações correspondentes às variações da elasticidade para valores não fisiológicos da densidade e da viscosidade do fluido bem como para valores mais condizentes com os fisiológicos são apresentadas.

\subsection{Simulação de um alvéolo}

Testou-se o modelo para expansão de um único segmento - Figura 3.7 - com $R_{m}=$

$10^{5} \frac{\mathrm{g}}{\mathrm{cm}^{3} \cdot \mathrm{s}}, \bar{P}_{m}=10^{3} \frac{\mathrm{g}}{\mathrm{cm} \cdot \mathrm{s}^{2}}, h_{\circ}=\frac{1}{32}$ e nove fontes e sorvedouros em uma malha computacional $32 \times 32$.

\subsubsection{Densidade e viscosidade não fisiológicos}

Empregando-se $\rho=1 \frac{\mathrm{g}}{\mathrm{cm}^{3}}$ e $\mu=10^{-2} \frac{\mathrm{g}}{\mathrm{cm} \cdot \mathrm{s}}$, algumas funções dentre as testadas produziram uma variação de área após $t=1.0 \mathrm{~s}$ e $t=1.5 \mathrm{~s}$ próxima às estimativas fornecidas pelas expressões (2.1) e (2.2). Entre elas a função

$$
k^{*}[i]=\operatorname{sen}\left(e^{k[i] n \Delta t}\right)
$$

que gera uma expansão poliédrica do segmento $\overline{B D}$ - Figura 5.1 - com formação de vórtices. 

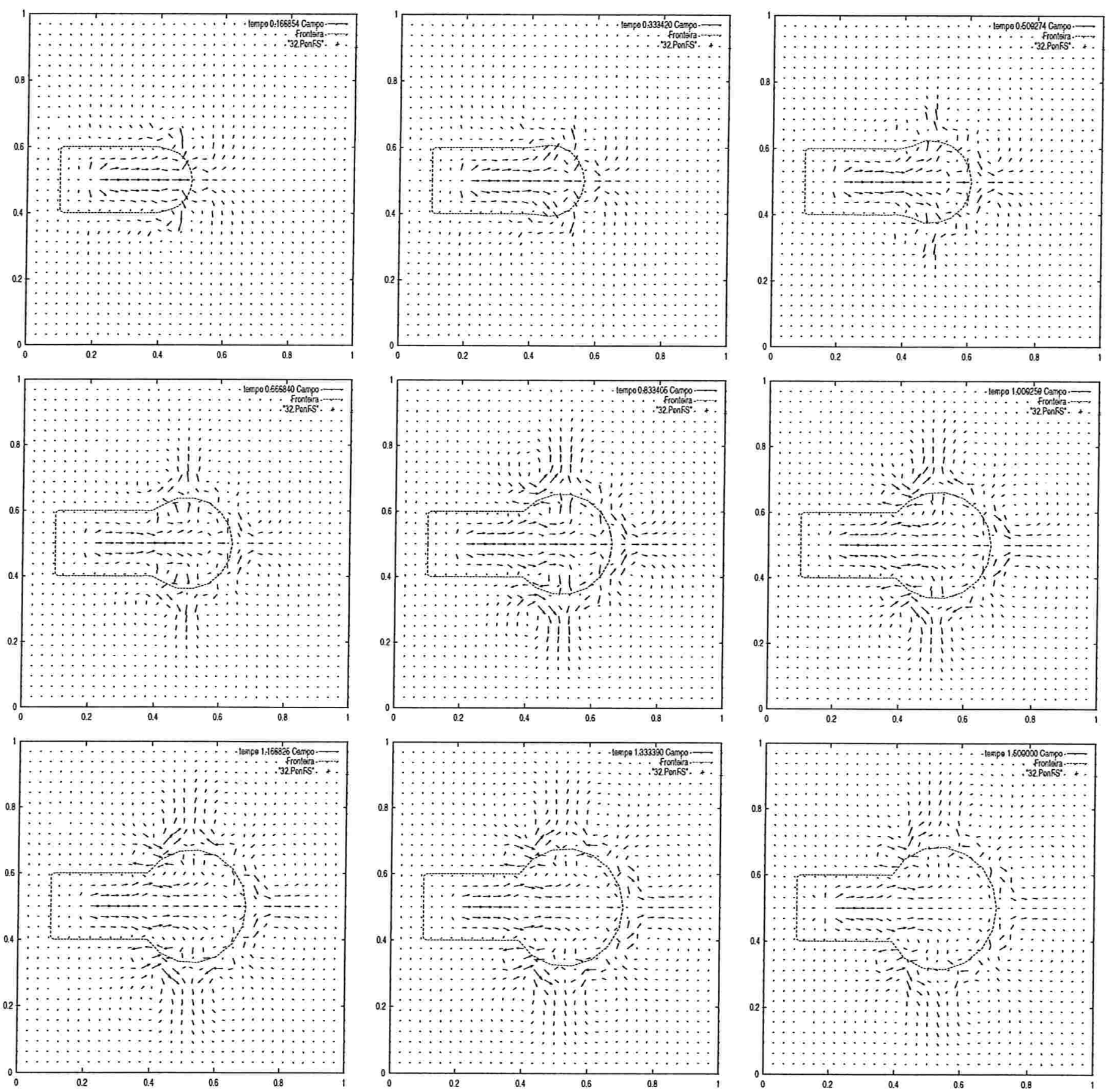

Figura 5.1: $k^{*}[i]=\operatorname{sen}\left(e^{k[i] n \Delta t}\right), R_{m}=10^{5} \frac{g}{\mathrm{~cm}^{3} \cdot s}, \bar{P}_{m}=10^{3} \frac{g}{\mathrm{~cm} \cdot \mathrm{s}^{2}}, h_{o}=\frac{1}{32}, \rho=1 \frac{g}{\mathrm{~cm}^{3}}$, $\mu=10^{-2} \frac{g}{\mathrm{~cm} . s}, k=9, \quad N=32$, Variação de área: $125.41 \%$ após $t=1.0 \mathrm{~s}, 156.34 \%$ após $t=1.5 \mathrm{~s}$, Fontes centradas em $(0.2,0.5)$, Sorvedouros centrados em $(0.55,0.1)(0.65,0.15)$ $(0.75,0.2)(0.85,0.3)(0.9,0.5)(0.85,0.7)(0.75,0.8)(0.65,0.85)(0.55,0.9)$ 
Com o reposicionamento dos sorvedouros pode-se ajustar a variação de área para os valores desejados. Para o teste anterior, por exemplo, centrando-se os sorvedouros nos pontos $(0.45,0.1),(0.6,0.1),(0.75,0.15),(0.85,0.3),(0.9,0.5),(0.85,0.7),(0.75,0.85)$, $(0.6,0.9)$ e $(0.45,0.9)$, obtém-se variações de área da ordem de $125.19 \%$ e $156.13 \%$ para $t=1.0 \mathrm{~s}$ e $t=1.5 \mathrm{~s}$, respectivamente. Dessa forma, o pequeno deslocamento dos centros de alguns dos nove sorvedouros determina uma pequena alteração na variação de área.

A ação da função

$$
k^{*}[i]=\operatorname{sen}\left(e^{k[i] n \Delta t}\right)
$$

na variação da elasticidade produz uma variação de área próxima a da obtida utilizando-se

$$
k[i]=1.0
$$

constante para todos os pontos do segmento $\overline{B D}$ no decorrer do tempo. Sabe-se que, quanto menor for essa constante, maior será a expansão verificada no instante final de integração das equações que regem o comportamento do sistema fluido-fibra. Isto é comprovado nas Figuras 5.2 e 5.3 , as quais representam testes para $k[i]=1.0$ e $k[i]=0.1$, respectivamente. 

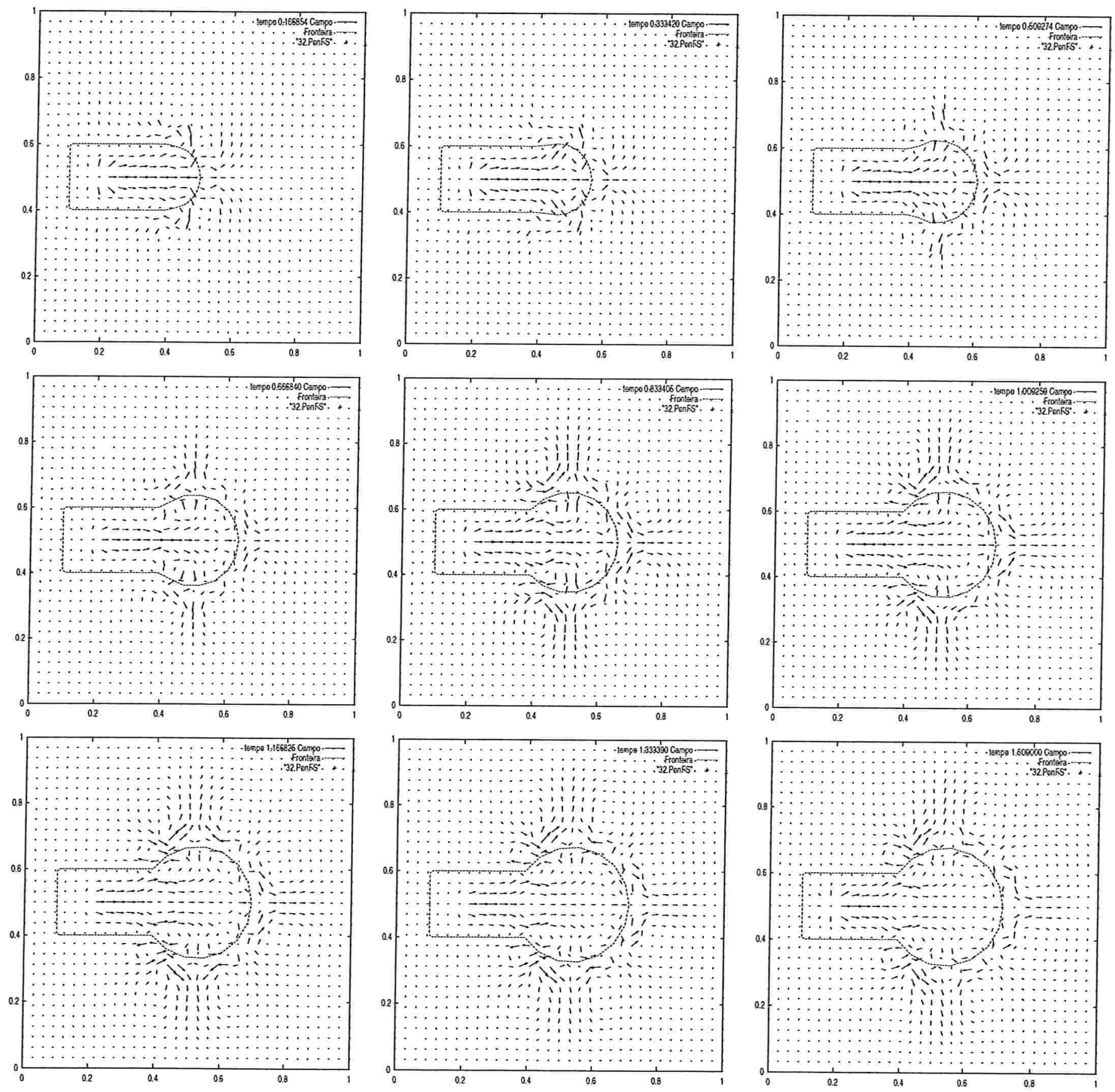

Figura 5.2: $k[i]=1.0, R_{m}=10^{5} \frac{g}{\mathrm{~cm}^{3} . \mathrm{s}}, \bar{P}_{m}=10^{3} \frac{\mathrm{g}}{\mathrm{cm} \cdot \mathrm{s}^{2}}, \quad h_{o}=\frac{1}{32}, \rho=1 \frac{\mathrm{g}}{\mathrm{cm}^{3}}, \mu=$ $10^{-2} \frac{g}{c m . s}, k=9, N=32$, Variação de área: $125 \%$ após $t=1.0 \mathrm{~s}, 154.98 \%$ após $t=1.5 s$, Fontes centradas em $(0.2,0.5)$, Sorvedouros centrados em $(0.55,0.1)(0.65,0.15)(0.75,0.2)$ $(0.85,0.3)(0.9,0.5)(0.85,0.7)(0.75,0.8)(0.65,0.85)(0.55,0.9)$ 

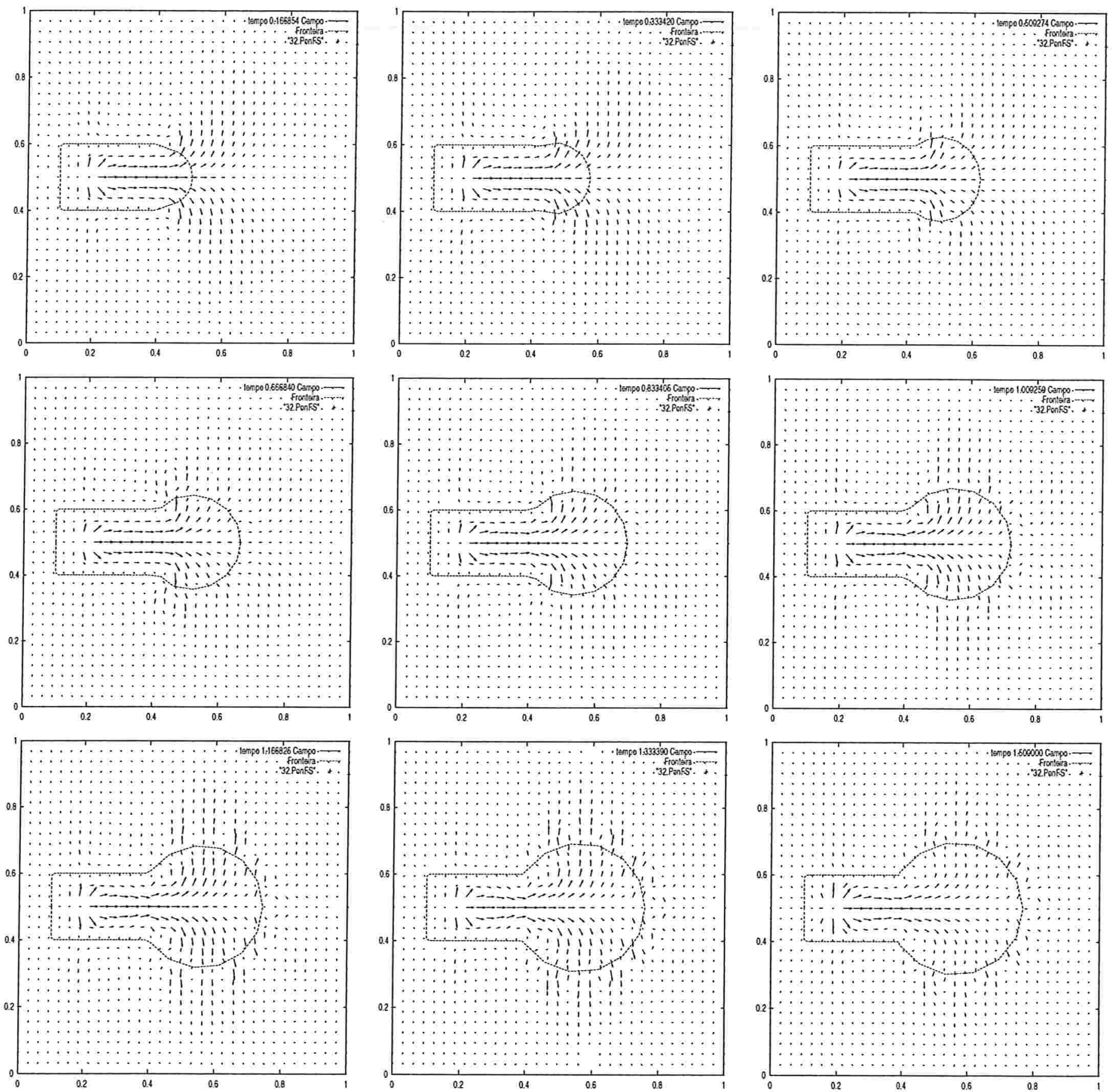

Figura 5.3: $k[i]=0.1, \quad R_{m}=10^{5} \frac{\mathrm{g}}{\mathrm{cm}^{3} \cdot s}, \bar{P}_{m}=10^{3} \frac{\mathrm{g}}{\mathrm{cm} \cdot \mathrm{s}^{2}}, \quad h_{o}=\frac{1}{32}, \rho=1 \frac{\mathrm{g}}{\mathrm{cm}^{3}}, \mu=$ $10^{-2} \frac{g}{c m . s}, k=9, N=32$, Variação de área: $148.86 \%$ após $t=1.0 s, 197.32 \%$ após $t=1.5 \mathrm{~s}$, Fontes centradas em $(0.2,0.5)$, Sorvedouros centrados em $(0.55,0.1)(0.65,0.15)(0.75,0.2)$ $(0.85,0.3)(0.9,0.5)(0.85,0.7)(0.75,0.8)(0.65,0.85)(0.55,0.9)$ 
O emprego de funções que elevam repentinamente os valores da elasticidade determina redução na variação de área.

A função

$$
k^{*}[i]=e^{(3.6) k[i] n \Delta t}
$$

induz aumento na variação de área até $t \approx 1.0 \mathrm{~s}$ e redução no intervalo de tempo restante, como ilustra a Figura 5.4. Assim, funções como essa podem ser utilizadas na simulação da expansão alveolar durante a inspiração e do recuo da parede alveolar durante a expiração. 

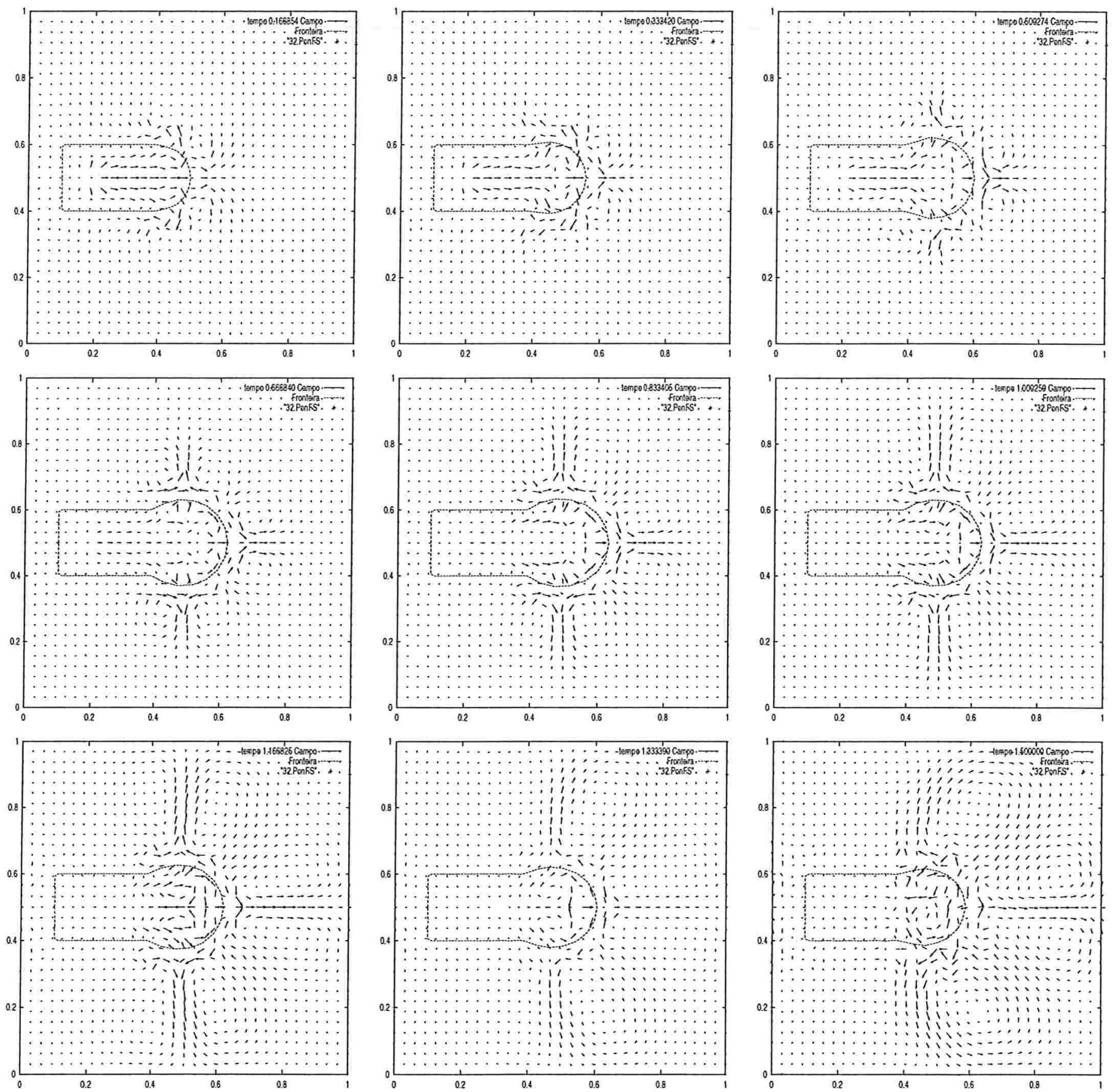

Figura 5.4: $k^{*}[i]=e^{(3.6) k[i] n \Delta t}, R_{m}=10^{5} \frac{g}{\mathrm{~cm}^{3} \cdot s}, \bar{P}_{m}=10^{3} \frac{g}{\mathrm{~cm} \cdot \mathrm{s}^{2}}, \quad h_{o}=\frac{1}{32}, \rho=1 \frac{\mathrm{g}}{\mathrm{cm}^{3}}$, $\mu=10^{-2} \frac{g}{c m . s}, k=9, \quad N=32$, Variação de área: $84.36 \%$ após $t=1.0 \mathrm{~s}, 60.18 \%$ após $t=1.5 s$, Fontes centradas em $(0.2,0.5)$, Sorvedouros centrados em $(0.55,0.1)(0.65,0.15)$ $(0.75,0.2)(0.85,0.3)(0.9,0.5)(0.85,0.7)(0.75,0.8)(0.65,0.85)(0.55,0.9)$ 


\subsubsection{Densidade e viscosidade fisiológicos}

Empregando-se $\rho=1.21 \times 10^{-3} \frac{\mathrm{g}}{\mathrm{cm}^{3}}$ e $\mu=1.9 \times 10^{-4} \frac{\mathrm{g}}{\mathrm{cm} . \mathrm{s}}$, valores para a densidade e a viscosidade do fluido mais próximos dos valores fisiológicos, a função

$$
k^{*}[i]= \begin{cases}(k[i] n \Delta t-5)^{2}, & \text { se } 0<n \Delta t \leq 1.0 \\ (k[i] n \Delta t-10)^{2}, & \text { se } 1.0<n \Delta t \leq 1.5\end{cases}
$$

foi a que determinou entre as funções testadas uma variação de área mais condizente com a desejada - Figura 5.5. Nesses testes foi necessário reduzir o tamanho do suporte $h_{o}$ e refinar a malha computacional. 

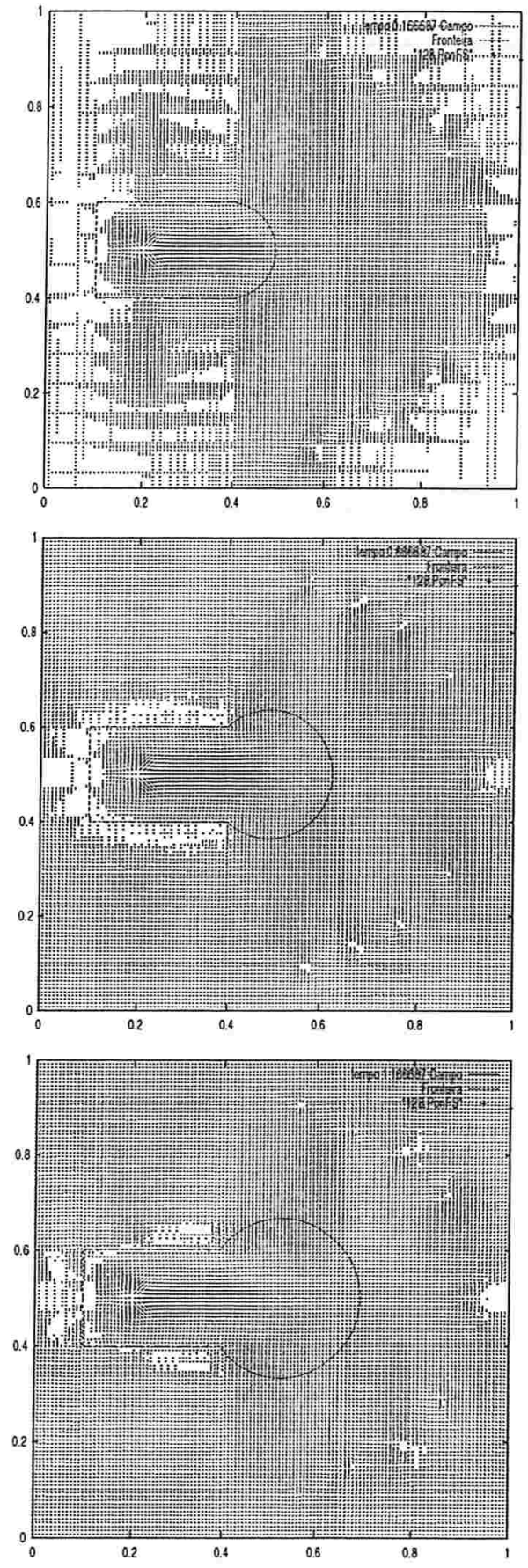
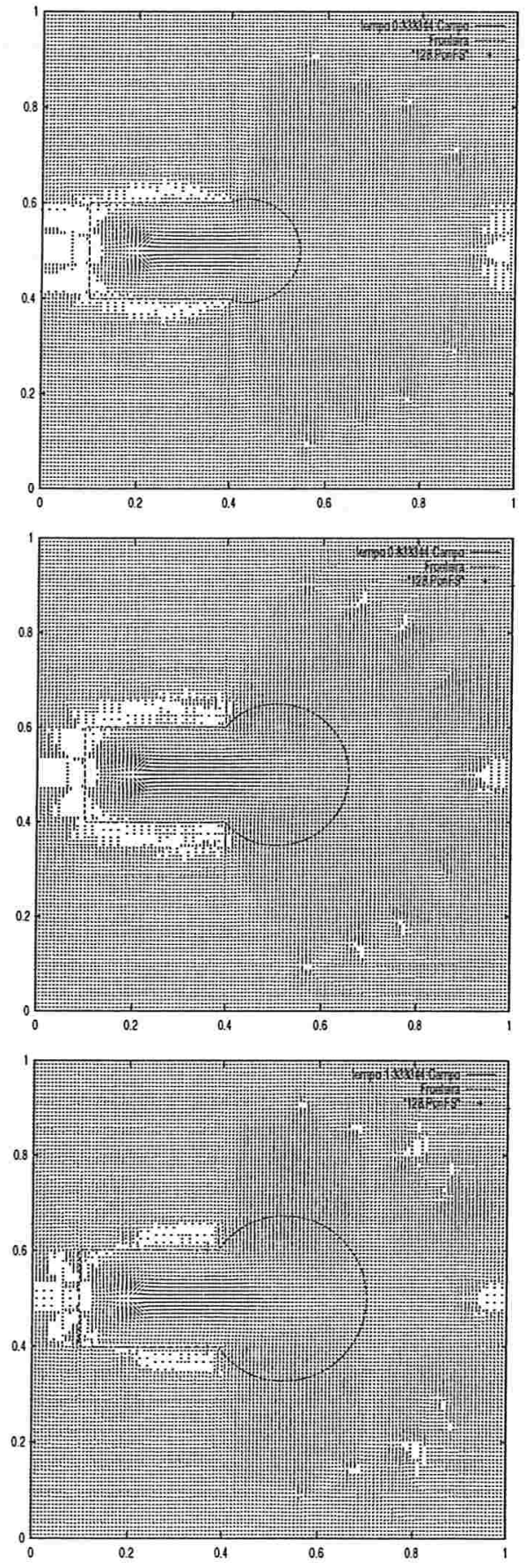
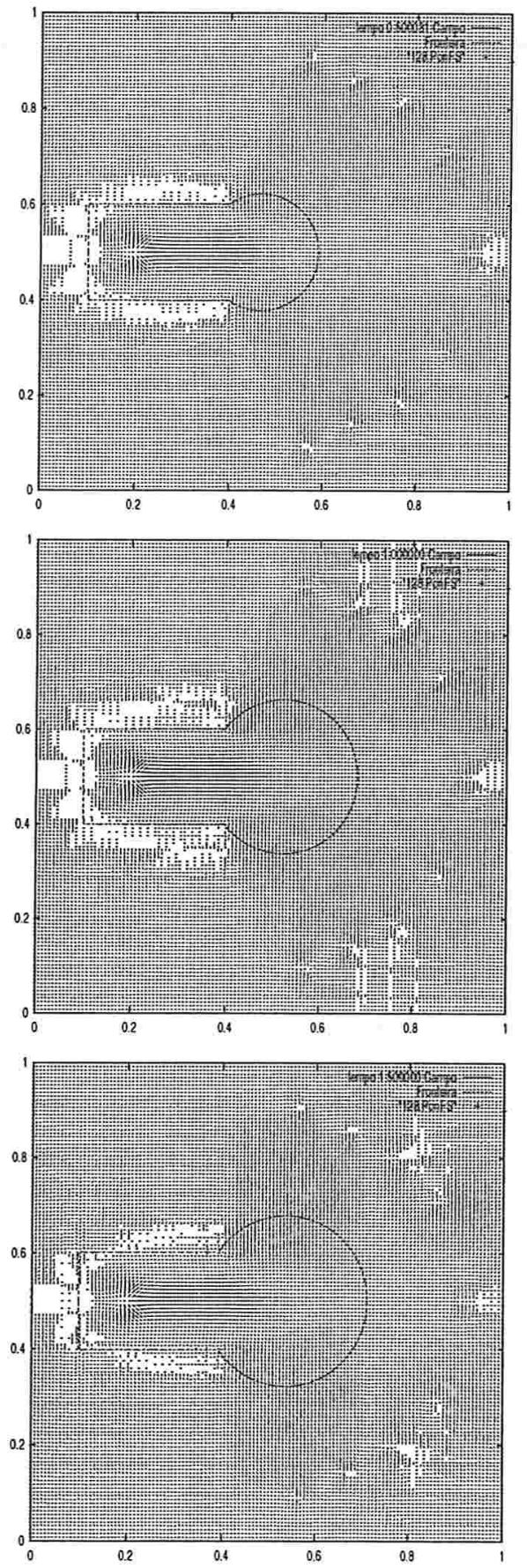

Figura 5.5: $(k[i] n \Delta t-5)^{2}$ para $0<n \Delta t \leq 1.0,(k[i] n \Delta t-10)^{2}$ para $1.0<n \Delta t \leq 1.5, R_{m}=10^{5} \frac{g}{\mathrm{~cm}^{3} \cdot s}$, $\bar{P}_{m}=10^{3} \frac{g}{c m . s^{2}}, h_{o}=\frac{1}{64}, \rho=1.21 \times 10^{-3} \frac{g}{\mathrm{~cm}^{3}}, \mu=1.9 \times 10^{-4} \frac{g}{\mathrm{~cm} \cdot s}, k=9, N=128$, Variação de área: $128.76 \%$ após $t=1.0 \mathrm{~s}, 157.28 \%$ após $t=1.5 \mathrm{~s}$, Fontes centradas em $(0.2,0.5)$, Sorvedouros centrados em $(0.55,0.1)(0.65,0.15)(0.75,0.2)(0.85,0.3)(0.9,0.5)(0.85,0.7)(0.75,0.8)(0.65,0.85)(0.55,0.9)$ 


\subsection{Simulação de dois alvéolos}

Testou-se o modelo para expansão de dois segmentos - Figura 4.7 - com $\rho=1 \frac{\mathrm{g}}{\mathrm{cm}^{3}}$, $\mu=10^{-2} \frac{\mathrm{g}}{\mathrm{cm} \cdot \mathrm{s}}, R_{m}=10^{5} \frac{\mathrm{g}}{\mathrm{cm}^{3} . \mathrm{s}}, \bar{P}_{m}=10^{3} \frac{\mathrm{g}}{\mathrm{cm} \cdot \mathrm{s}^{2}}, h_{o}=\frac{1}{32}$ e nove fontes e sorvedouros em uma malha computacional $32 \times 32$.

\subsubsection{Expansões simétricas}

Na expansão simultânea de dois segmentos, verificou-se que a variação de área é muito menor do que a desejada, principalmente quando se analisa a expansão de cada um dos segmentos separadamente. As Figuras 5.6, 5.7 e 5.8 evidenciam essa expansão quando se usa uma função para a variação temporal ou valores fixos para a elasticidade ao longo do tempo.

Parâmetros utilizados na expansão de um único segmento já não produzem resultados satisfatórios quando aplicados ao modelo para duas expansões simultâneas. Isto é exemplificado no teste ilustrado pela Figura 5.6. Os dados usados no referido teste não produzem uma variação de área adequada, contrariamente ao que ocorre quando se emprega esse mesmo conjunto de dados para simular a expansão de um único segmento Figura 5.1. 

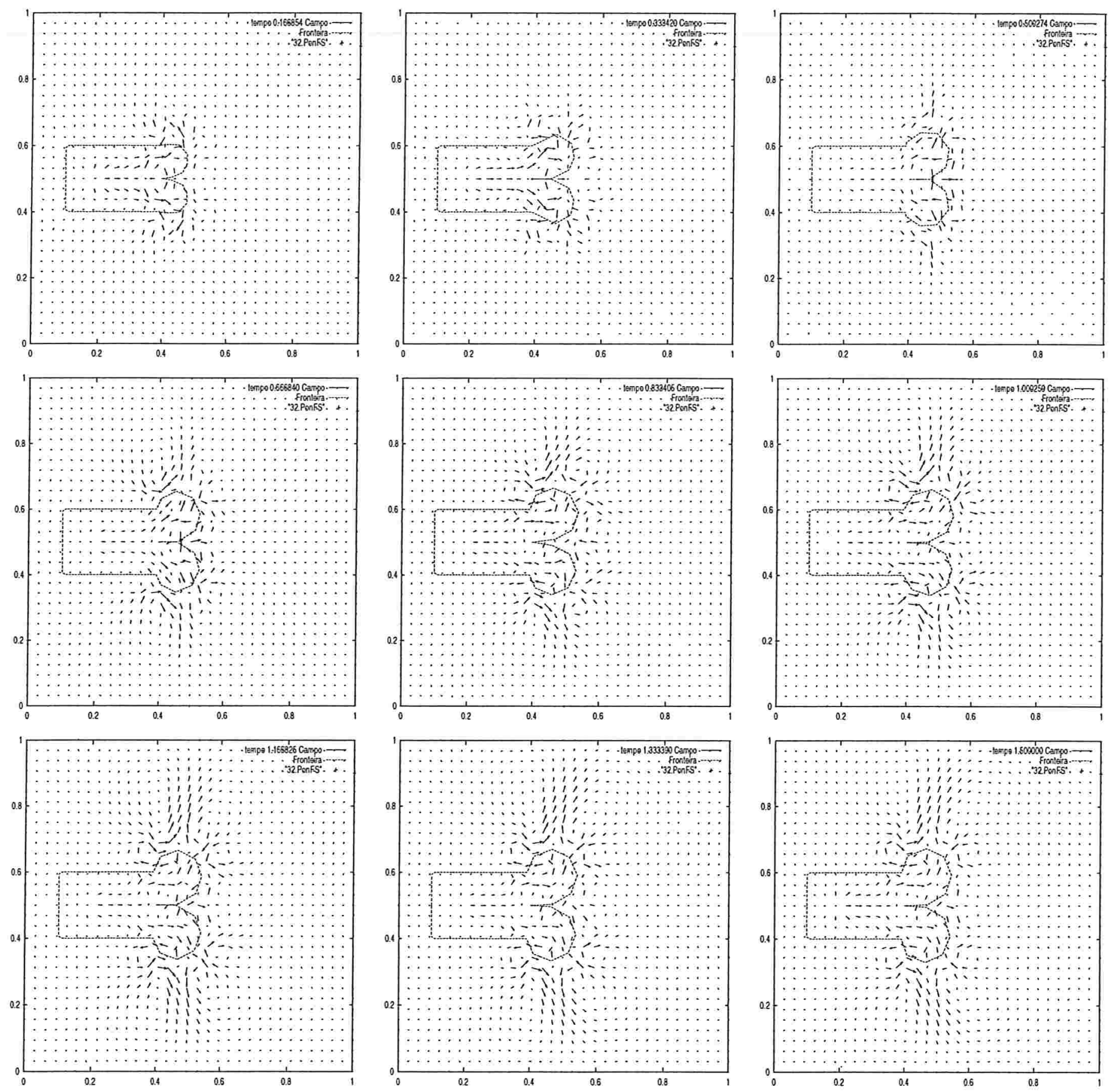

Figura 5.6: $\quad k^{*}[i]=\operatorname{sen}\left(e^{k[i] n \Delta t}\right), R_{m}=10^{5} \frac{g}{\mathrm{~cm}^{3} \cdot s}, \bar{P}_{m}=10^{3} \frac{\mathrm{g}}{\mathrm{cm} \cdot \mathrm{s}^{2}}, h_{o}=\frac{1}{32}, \rho=1 \frac{\mathrm{g}}{\mathrm{cm}}$, $\mu=10^{-2} \frac{g}{\mathrm{~cm} . s}, k=9, \quad k=32$, Variação de área: $60.57 \%$ após $t=1.0 \mathrm{~s}, 64.99 \%$ após $t=1.5 \mathrm{~s}$, Fontes centradas em $(0.2,0.5)$, Sorvedouros centrados em $(0.55,0.1)(0.65,0.15)$ $(0.75,0.2)(0.85,0.3)(0.9,0.5)(0.85,0.7)(0.75,0.8)(0.65,0.85)(0.55,0.9)$ 

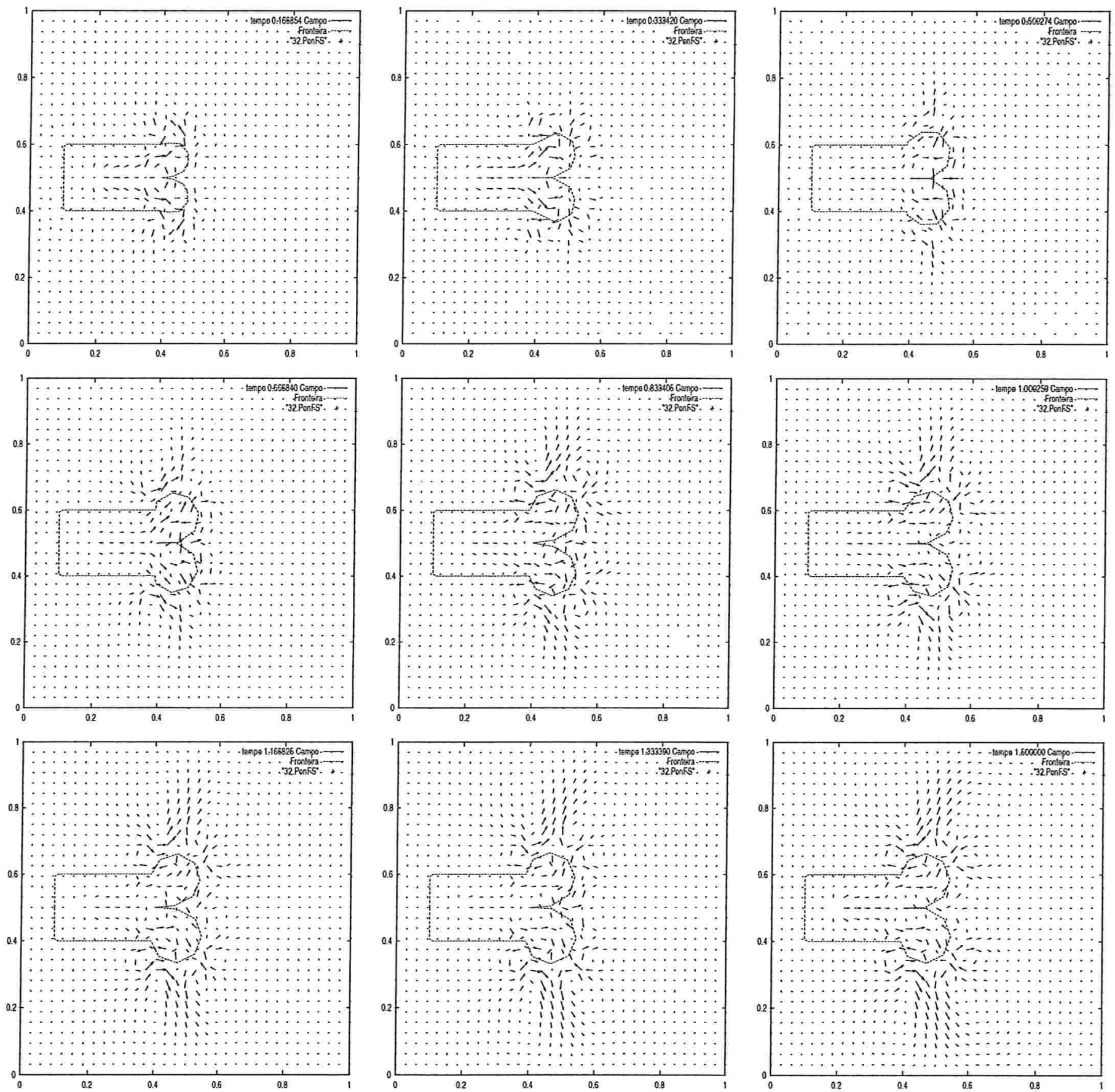

Figura 5.7: $k[i]=1.0, \quad R_{m}=10^{5} \frac{\mathrm{g}}{\mathrm{cm}^{3} \cdot s}, \bar{P}_{m}=10^{3} \frac{\mathrm{g}}{\mathrm{cm} \cdot \mathrm{s}^{2}}, \quad h_{o}=\frac{1}{32}, \rho=1 \frac{\mathrm{g}}{\mathrm{cm}^{3}}, \mu=$ $10^{-2} \frac{g}{c m . s}, k=9, \quad N=32$, Variação de área: $59.98 \%$ após $t=1.0 s, 62.46 \%$ após $t=1.5 s$, Fontes centradas em $(0.2,0.5)$, Sorvedouros centrados em $(0.55,0.1)(0.65,0.15)(0.75,0.2)$ $(0.85,0.3)(0.9,0.5)(0.85,0.7)(0.75,0.8)(0.65,0.85)(0.55,0.9)$ 

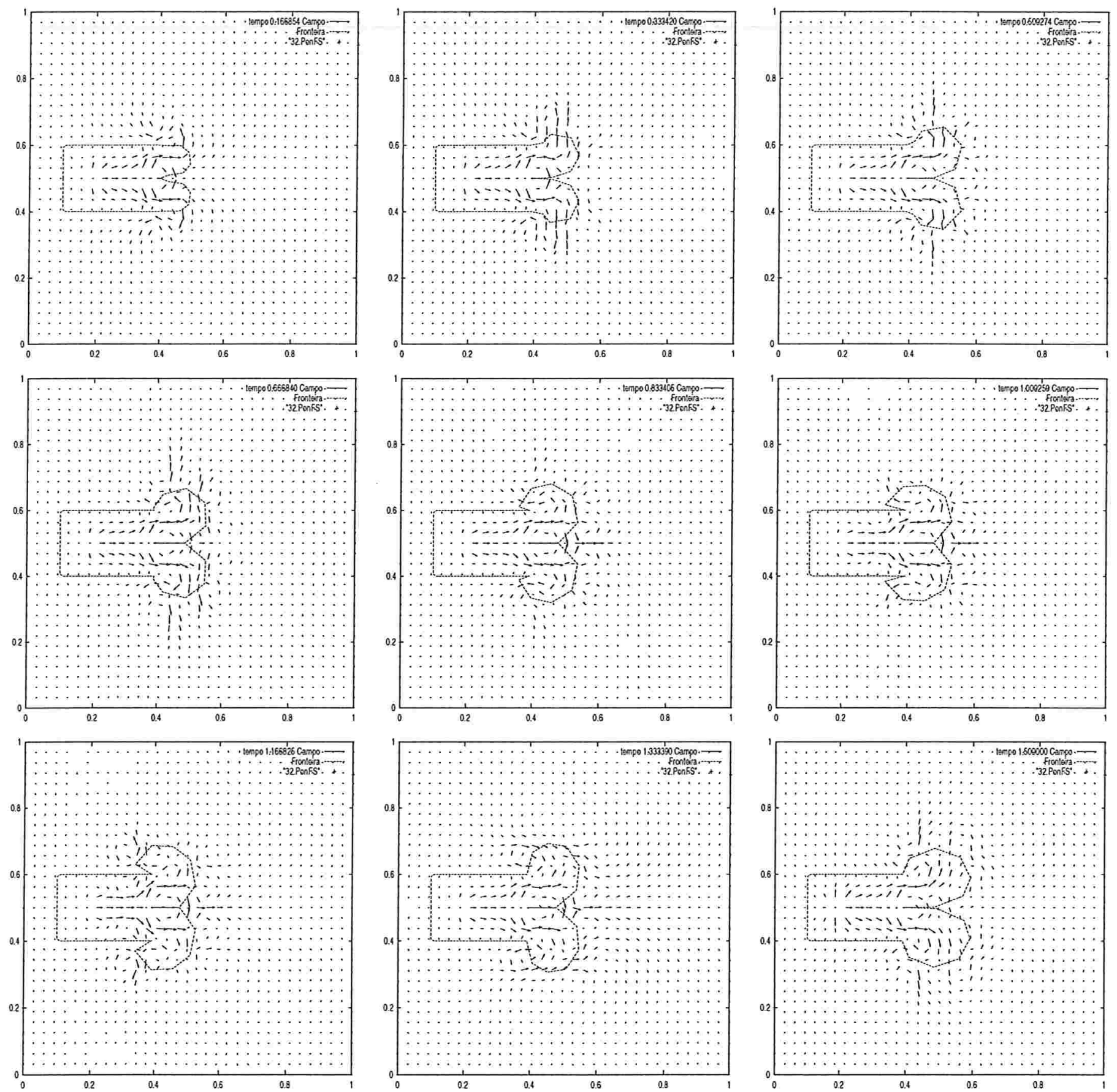

Figura 5.8: $k[i]=0.1, R_{m}=10^{5} \frac{\mathrm{g}}{\mathrm{cm}^{3} \cdot \mathrm{s}}, \bar{P}_{m}=10^{3} \frac{\mathrm{g}}{\mathrm{cm} \cdot \mathrm{s}^{2}}, \quad h_{o}=\frac{1}{32}, \rho=1 \frac{\mathrm{g}}{\mathrm{cm}^{3}}, \mu=$ $10^{-2} \frac{\mathrm{g}}{\mathrm{cm} . \mathrm{s}}, \quad k=9, \quad N=32$, Variação de área: $72.63 \%$ após $t=1.0 \mathrm{~s}, 90.88 \%$ após $t=1.5 \mathrm{~s}$, Fontes centradas em $(0.2,0.5)$, Sorvedouros centrados em $(0.55,0.1)(0.65,0.15)(0.75,0.2)$ $(0.85,0.3)(0.9,0.5)(0.85,0.7)(0.75,0.8)(0.65,0.85)(0.55,0.9)$ 
Uma maior expansão no modelo para dois alvéolos pode ser verificada quando se modifica alguns parâmetros. $\mathrm{O}$ aumento do tamanho do suporte $h_{o}$ das fontes e sorvedouros, o emprego de funções que atribuem pequenos valores à elasticidade na evolução temporal, o reposicionamento de fontes e sorvedouros e a introdução de um número maior de fontes e sorvedouros $(k>9)$ podem aumentar a variação de área.

Com os testes ilustrados pelas Figuras 5.6, 5.7 e 5.8, fica evidente que é necessário testar novos parâmetros que ajustem o modelo matemático para dois alvéolos ao modelo real. Principalmente, é preciso repensar a forma como a força exercida pelo ponto $E$ é modelada.

\subsubsection{Expansões assimétricas}

As Figuras 5.9 e 5.10 evidenciam a simulação de duas expansões diferenciadas através do emprego de dois valores para $k[i]$ ou de duas funções para a variação temporal da elasticidade associada aos pontos dos segmentos $\overline{B E}$ e $\overline{D E}$. Ao se comparar essas duas figuras, observa-se que o uso de uma função para a variação temporal pode possibilitar expansões menos disformes do que aquelas verificadas quando se emprega um valor fixo para a elasticidade. 

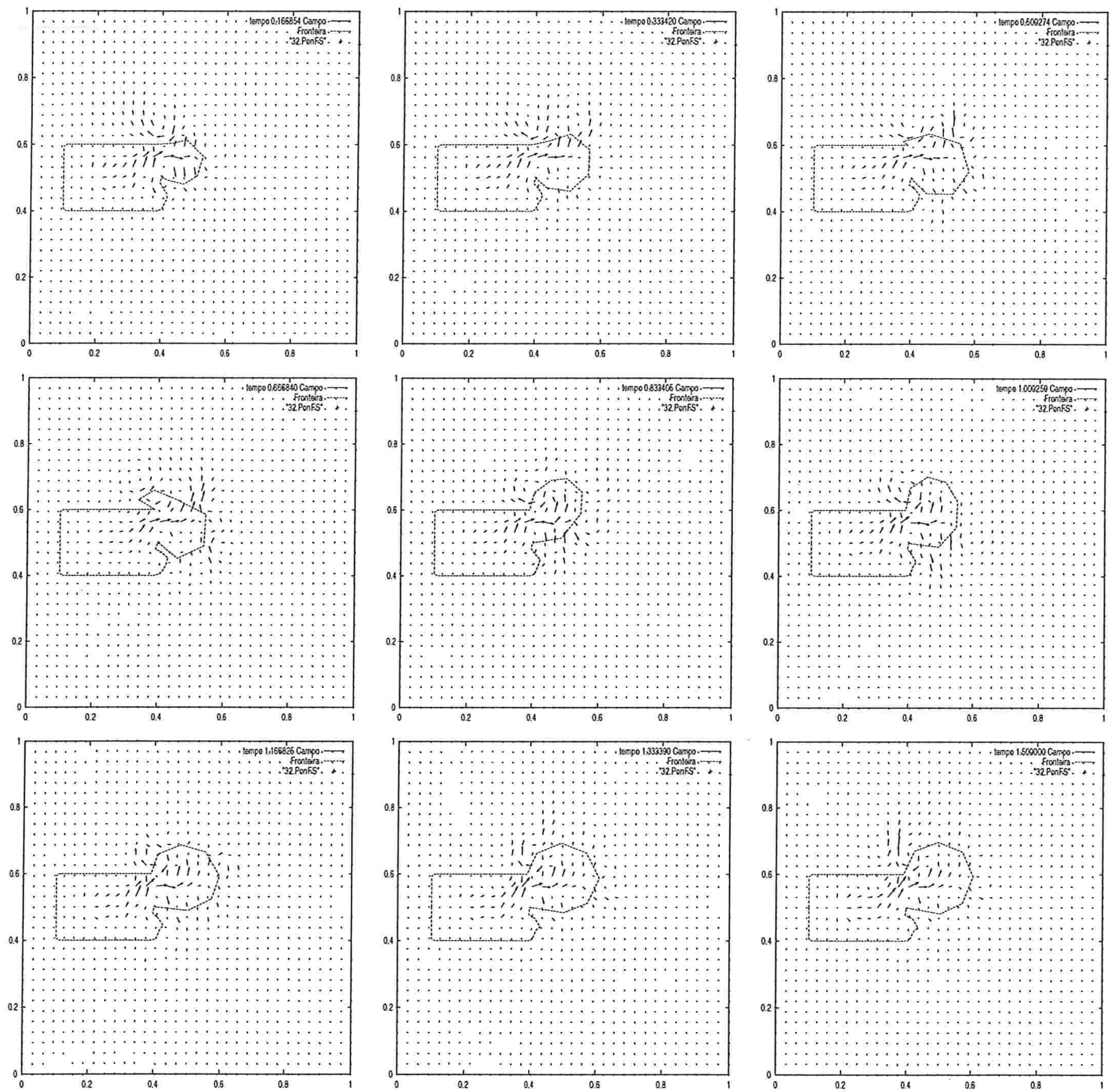

Figura 5.9: $k[i]=0.1$ no segmento $\overline{B E}$ e $k[i]=1.0$ no segmento $\overline{D E}, R_{m}=10^{5} \frac{\mathrm{g}}{\mathrm{cm}^{3} . \mathrm{s}}, \bar{P}_{m}=$ $10^{3} \frac{g}{\mathrm{~cm} \cdot \mathrm{s}^{2}}, h_{o}=\frac{1}{32}, \rho=1 \frac{\mathrm{g}}{\mathrm{cm}^{3}}, \mu=10^{-2} \frac{\mathrm{g}}{\mathrm{cm} \cdot \mathrm{s}}, k=9, N=32$, Variação de área: $46.39 \%$ após $t=1.0 \mathrm{~s}, 60.36 \%$ após $t=1.5 \mathrm{~s}$, Fontes centradas em $(0.2,0.5)$, Sorvedouros centrados em $(0.55,0.1)(0.65,0.15)(0.75,0.2)(0.85,0.3)(0.9,0.5)(0.85,0.7)(0.75,0.8)(0.65,0.85)(0.55,0.9)$ 

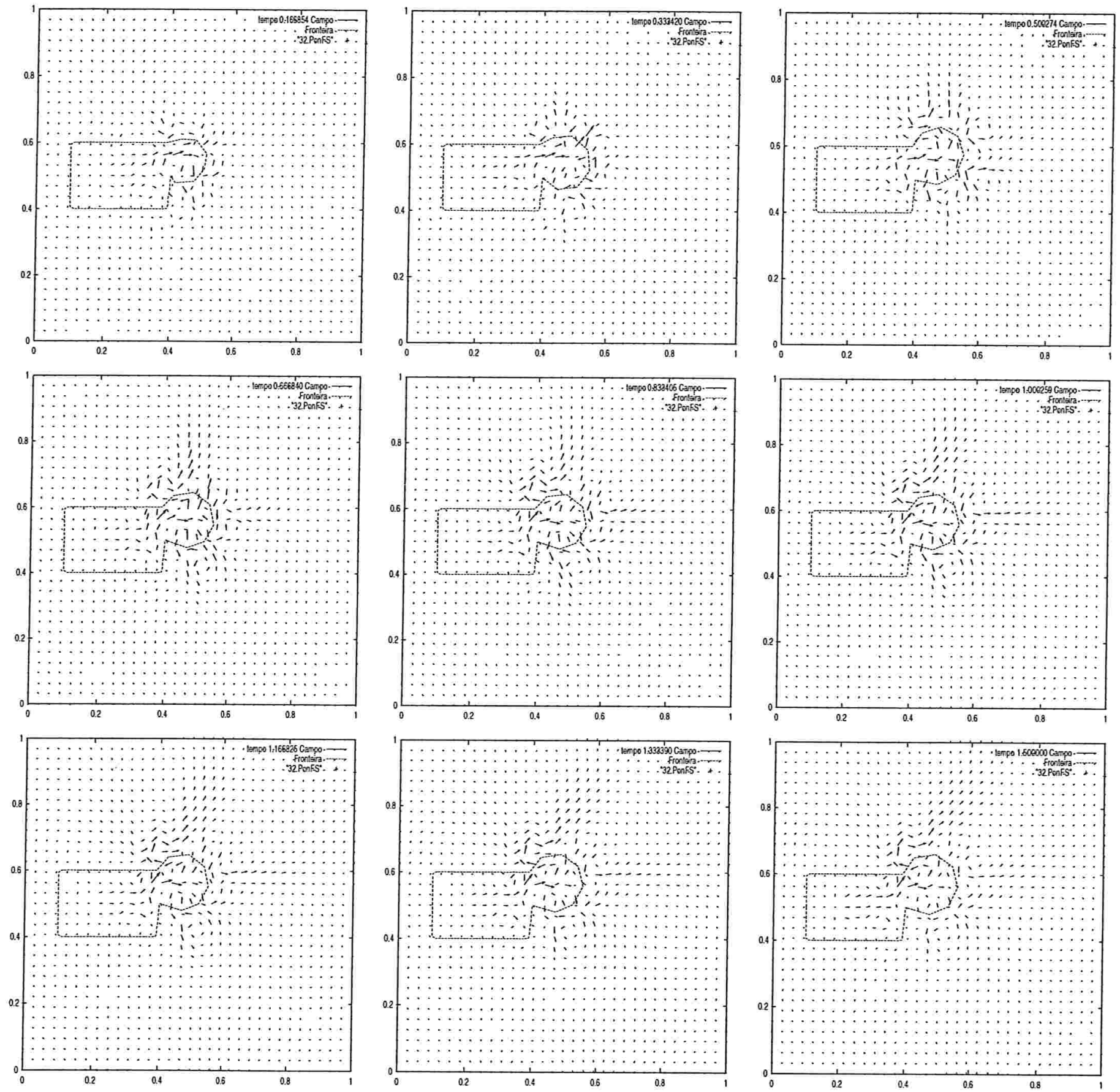

Figura 5.10: $k^{*}[i]=\operatorname{sen}\left(e^{k[i] n \Delta t}\right)$ no segmento $\overline{B E}$ e $k^{*}[i]=(k[i] n \Delta t-10)^{2}$ no segmento $\overline{D E}$, $R_{m}=10^{5} \frac{\mathrm{g}}{\mathrm{cm}^{3} \cdot s}, \bar{P}_{m}=10^{3} \frac{\mathrm{g}}{\mathrm{cm} \cdot \mathrm{s}^{2}}, h_{o}=\frac{1}{32}, \rho=1 \frac{\mathrm{g}}{\mathrm{cm}^{3}}, \mu=10^{-2} \frac{\mathrm{g}}{\mathrm{cm} \cdot \mathrm{s}}, k=9, N=32$, Variação de área: $33.07 \%$ após $t=1.0 s, 37.54 \%$ após $t=1.5 \mathrm{~s}$, Fontes centradas em $(0.2,0.5)$, Sorvedouros centrados em $(0.55,0.1)(0.65,0.15)(0.75,0.2)(0.85,0.3)(0.9,0.5)(0.85,0.7)(0.75,0.8)(0.65,0.85)(0.55,0.9)$ 


\section{Conclusão}

Neste trabalho modelou-se a elasticidade de uma fibra no decorrer do tempo atráves do método da fronteira imersa estruturado por Peskin. A expansão sofrida pelo alvéolo pulmonar durante a inspiração foi empregada como base para as simulações e essa expansão foi controlada pela variação de área estimada a partir da variação volumétrica do alvéolo. Mostrou-se assim que a expansão sofrida pela fronteira imersa devido às suas propriedades elásticas pode ser modelada para se obter uma determinada variação de área. Para tanto, alguns parâmetros precisam ser ajustados, dentre os quais se destacam a função $k^{*}[i]$ para a evolução temporal dos valores $k[i]$ abribuídos à elasticidade, o número $k$ de fontes e sorvedouros a ser introduzido no modelo assim como o posicionamento destes, os valores da pressão $\bar{P}_{m}$ e da resistência $R_{m}$ associados a cada fonte e sorvedouro, o suporte $h_{o}$ das funções que definem a função peso $\Psi_{m}(x)$, a qual especifica a distribuição espacial da m-ésima fonte e do m-ésimo sorvedouro, e o tamanho da malha utilizada no método computacional.

A continuidade do trabalho requer o conhecimento de valores fisiológicos para a densidade e a viscosidade do fluido como também para a pressão $\bar{P}_{m}$ e a resistência $R_{m}$ associadas às fontes e aos sorvedouros. Ao invés de se manter $\bar{P}_{m}$ e $R_{m}$ constantes, estratégia aqui adotada, seus valores poderiam ser distintos, isto é, $\bar{P}_{1} \neq \bar{P}_{2} \neq \ldots \neq \bar{P}_{m}$ e $R_{1} \neq R_{2} \neq \ldots \neq R_{m}$, variando inclusive com o tempo. Ter-se-ia também que trabalhar com dois fluidos de características distintas, já que no modelo real para o mecanismo respiratório existe ar dentro do alvéolo e sangue fora dele. Como o modelo exige o emprego de malhas refinadas para valores fisiológicos da densidade $\rho$ e viscosidade $\mu$, o uso de uma malha adaptativa como a proposta por Roma [31] reduziria o tempo de execução e 
conseqüentemente o custo computacional. A variação dos valores relacionados à elasticidade poderia ser espacial além de temporal, ou seja, associar-se-ia a $k[i]$ a posição no instante de tempo imediatamente anterior, e não sempre a posição na configuração inicial da fronteira. Além disso, poder-se-ia pensar em fronteiras abertas, como por exemplo aquelas necessárias à simulação do fluxo sangüíneo atráves de uma válvula cardíaca, e na construção de um modelo matemático e computacional tridimensional. A modificação do domínio computacional $\Omega=[0,1] \mathrm{X}[0,1]$ permitiria a simulação de escoamentos em tubos mais longos.

O domínio da expansão da fronteira imersa bem como de sua geometria no decorrer do tempo poderia ser aplicado a um grande número de problemas, entre eles a simulação da locomoção de microorganismos e da expansão do balão inflável acoplado à ponta do catéter empregado na desobstrução de vasos sangüíneos (cateterismo). Ou ainda, a simulação da emissão de falsos pés, ou pseudopodes, pelos glóbulos brancos. Esta estratégia permite a essas células sangüíneas realizar a fagocitose, fenômeno que consiste em englobar para depois destruir organismos invasores. 


\section{Fluxo laminar e turbulento}

O fluxo de ar ocorre somente quando existe uma diferença de pressão: o gás se move de um região de pressão mais alta para uma região de pressão mais baixa.

Basicamente, existem dois tipos de fluxo: o laminar e o turbulento.

O fluxo laminar é suave e ordenado. Nele, as partículas que constituem o fluido se movem paralelamente às paredes de um tubo, como mostra a Figura A.1.

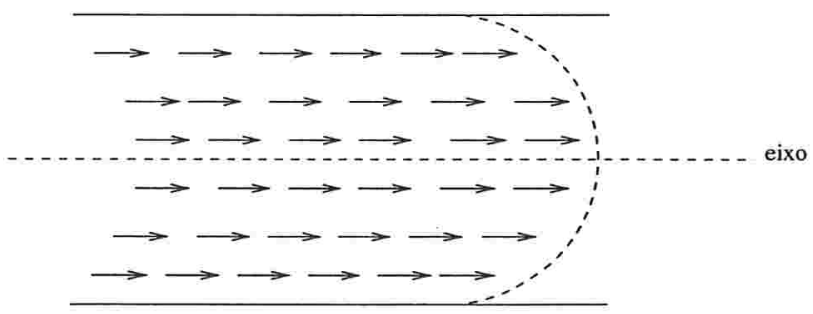

Figura A.1: Comportamento das partículas do fluido no escoamento laminar

As partículas do fluido próximas às paredes do tubo têm velocidade nula. A velocidade cresce à medida que a distância em relação à parede do tubo aumenta, atingindo valor máximo no eixo do tubo.

Quando um fluido escoa por um tubo, ele sempre perde energia ao longo do mesmo. A perda de energia se deve às forças de fricção e é caracterizada por uma queda de pressão. Essa queda de pressão é uma medida da resistência do tubo à passagem do fluido.

No fluxo laminar, a queda de pressão não pode ser atribuída às forças de atrito entre as partículas do fluido e as paredes do tubo, já que a velocidade das partículas próximas às paredes é nula. A queda de pressão ao longo do tubo durante o escoamento se deve à 
viscosidade do fluido.

A viscosidade de um fluido pode ser definida como a resistência interna do mesmo ao escoamento e é causada pelo atrito entre as suas moléculas. Diferentes fluidos têm diferentes viscosidades, que variam também com a temperatura. A viscosidade de líquidos diminui com o aumento da temperatura; a viscosidade de gases aumenta com o aumento da temperatura. A Tabela A.1 traz valores para a viscosidade do ar sob certas temperaturas.

\begin{tabular}{|c|c|c|}
\hline Temperatura $\left({ }^{\circ} \mathrm{C}\right)$ & $\mu_{\text {ar }}\left(\mu_{\mathbf{p}}\right)$ & $\mu_{\text {ar }}\left(\frac{\mathrm{g}}{\mathrm{cm} . \mathrm{s}}\right)$ \\
\hline 0 & 171 & $1.71 \times 10^{-4}$ \\
\hline 20 & 181 & $1.81 \times 10^{-4}$ \\
\hline 40 & 190 & $1.9 \times 10^{-4}$ \\
\hline
\end{tabular}

Tabela A.1: Viscosidade do ar - Sears [35]

OBS.:

1 poise $=1 \frac{d y n . s}{c m^{2}}$

$1 d y n=10^{-5} \mathrm{~N}=10^{-5} \frac{\mathrm{kg} \cdot \mathrm{m}}{\mathrm{s}^{2}}=1 \frac{\mathrm{g} \cdot \mathrm{cm}}{\mathrm{s}^{2}}$

1 poise $=1 \frac{\mathrm{g} \cdot \mathrm{cm}}{\mathrm{s}^{2}} \frac{\mathrm{s}}{\mathrm{cm} \mathrm{m}^{2}}=1 \frac{\mathrm{g}}{\mathrm{cm} \cdot \mathrm{s}}$

$1 \mu_{p}(1$ micropoise $)=10^{-6}$ poise $=10^{-6} \frac{g}{\mathrm{~cm} \cdot \mathrm{s}}$

O comportamento dos fluidos em escoamento laminar foi investigado pelo engenheiro civil alemão Heinrich Gotthilf Hagen e pelo fisiologista francês Jean Leonard Marie Poiseuille entre 1839 e 1841 . O estudo de ambos forneceu uma equação que rege o escoamento laminar de um fluido de viscosidade constante e independente da velocidade do mesmo, conhecida como a lei de Hagen-Poiseuille:

$$
\Delta P=P_{1}-P_{2}=\frac{8 \mu L}{\pi r^{4}} \dot{V}
$$

onde

$\Delta \mathrm{P}$ : variação da pressão entre dois pontos, medida em $\mathrm{cmH}_{2} \mathrm{O}$

$\mu$ : viscosidade do fluido

L : comprimento do tubo

$r$ : raio do tubo

$\dot{V}$ : vazão volumétrica de fluido, medida em $\ell / s$ 
No fluxo laminar, se a geometria do tubo e a viscosidade do gás forem constantes, a queda de pressão ao longo do tubo durante o escoamento será diretamente proporcional à vazão volumétrica de gás, ou seja

$$
\Delta P=K_{1} \dot{V}
$$

onde $K_{1}$ é uma constante de proporcionalidade que inclui o comprimento e o raio do tubo bem como a viscosidade do gás.

Acima de uma certa vazão volumétrica $\dot{V}$ ocorre a transição do fluxo laminar para o fluxo turbulento. Esse ponto é conhecido como sendo a taxa crítica de fluxo do fluido.

Em escoamentos turbulentos, as partículas do fluido não se deslocam paralelamente às paredes do tubo. Elas se movem desorganizadamente em relação à direção geral do escoamento, como ilustra a Figura A.2.

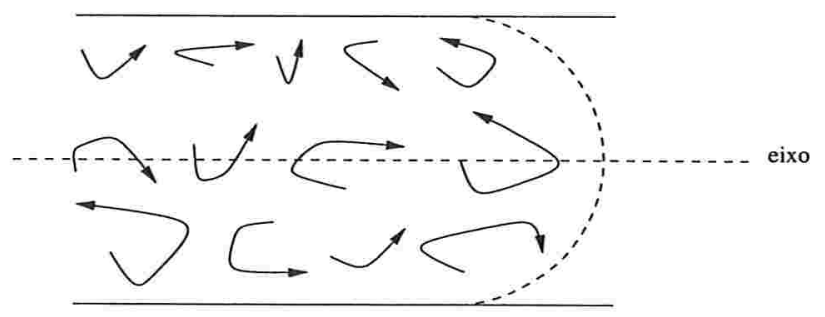

Figura A.2: Comportamento das partículas do fluido no escoamento turbulento

As partículas do fluido em escoamento turbulento se deslocam ao longo do tubo em velocidades uniformes; as partículas próximas às paredes do tubo têm a mesma velocidade daquelas localizadas no eixo do tubo.

Nos escoamentos turbulentos, a queda de pressão se deve também à densidade ou massa específica do fluido e ao atrito entre as partículas do fluido e as paredes do tubo, já que a velocidade das partículas próximas às paredes não é mais nula.

A densidade é definida como a razão entre a massa e o volume do fluido, isto é

$$
\rho=\frac{m}{V}
$$

e medida em $\mathrm{g} / \mathrm{cm}^{3}$, variando com a temperatura e a pressão. A densidade do ar, a uma temperatura de $0^{\circ} \mathrm{C}$ e pressão de 1 atmosfera, é de $1.293 \times 10^{-3} \frac{\mathrm{g}}{\mathrm{cm}^{3}}$; já a uma temperatura de $20^{\circ} \mathrm{C}$ e pressão de 1 atmosfera, é de $1.21 \times 10^{-3} \frac{\mathrm{g}}{\mathrm{cm}^{3}}$ - Halliday [15]. 
Nesse tipo de escoamento, a queda de pressão de um gás é diretamente proporcional ao quadrado da vazão volumétrica, ou seja

$$
\Delta P=K_{2} \dot{V}^{2}
$$

onde $K_{2}$ é uma constante de proporcionalidade que inclui o comprimento e o raio do tubo bem como a densidade e a viscosidade do gás.

Nas vias aéreas do sistema respiratório, o escoamento turbulento ocorre juntamente com o escoamento laminar. O fluxo turbulento é encontrado nas vias aéreas mais amplas, como a traquéia, enquanto que o fluxo laminar ocorre nas vias aéreas de menor diâmetro, como os bronquíolos terminais e respiratórios. A Figura A.3 mostra o tipo de fluxo normalmente existente nas vias aéreas do sistema respiratório humano.

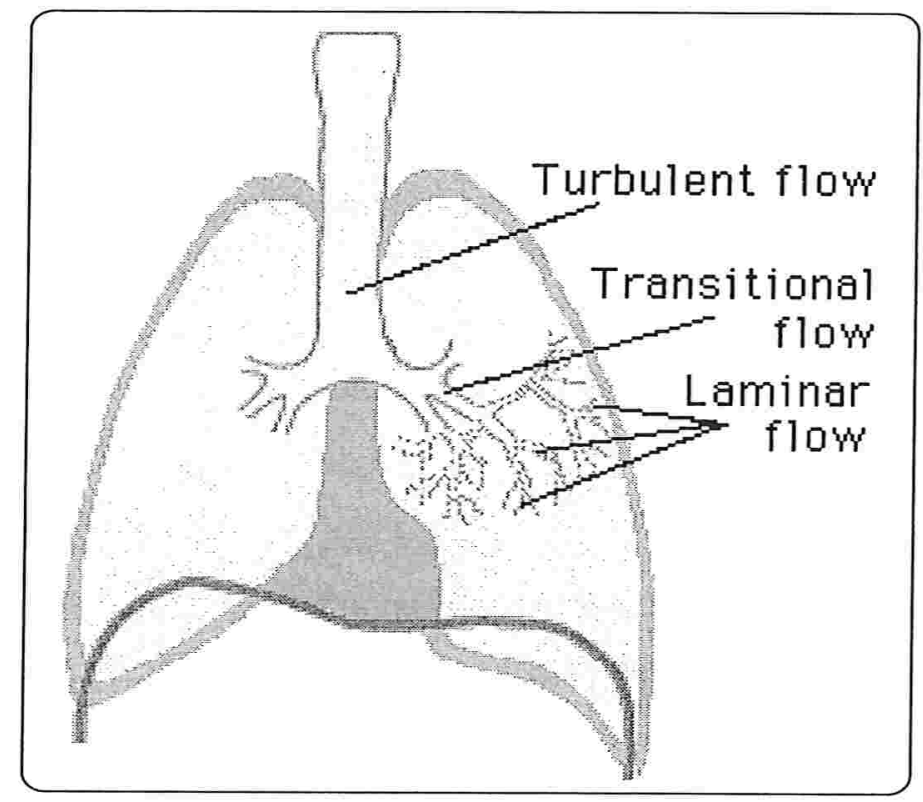

Figura A.3: Fluxo nas vias aéreas - Hopkins [17]

Os escoamentos laminar e turbulento podem ser combinados em uma única equação para expressar a queda de pressão ao longo de uma determinada via aérea:

$$
\Delta P=K_{1} \dot{V}+K_{2} \dot{V}^{2}
$$




\section{Número de Reynolds}

Para avaliar o escoamento de um fluido pode-se empregar um número adimensional, o número de Reynolds $\left(\mathbf{N}_{R}\right)$, definido por

$$
N_{R}=\frac{2 r \rho v}{\mu}
$$

onde

$v$ é a velocidade média do fluido

$\rho$ é a densidade do fluido

$\mu$ é a viscosidade do fluido e

$r$ é o raio do tubo.

Quando

$$
\begin{aligned}
& N_{R}<2000 \\
& N_{R}>3000 \\
& 2000 \leq N_{R} \leq 3000
\end{aligned}
$$

tem-se, respectivamente, escoamento laminar, escoamento turbulento e escoamento instável. Este último é o escoamento que pode passar de laminar para turbulento ou vice-versa.

A Tabela B.1 traz o número de Reynolds para escoamentos em algumas das vias aéreas do sistema respiratório. 


\begin{tabular}{|l|c|ll|}
\hline VIA AÉREA & DIÂMETRO $(\mathrm{mm})$ & $\dot{V}(\ell / s)-\mathbf{N}_{R}$ & \\
\hline Cavidade nasal & 5 & $0,333-400$ & $3,33-4000$ \\
\hline Faringe & 12 & $0,333-800$ & $3,33-8000$ \\
\hline Traquéia & 21 & $0,333-1250$ & $3,33-12500$ \\
\hline Brônquios & 17 & $0,333-910$ & $3,33-9100$ \\
\hline Bronquíolos & 9 & $0,333-700$ & $3,33-7000$ \\
\hline Bronquíolos & 6 & $0,333-570$ & $3,33-5700$ \\
\hline Bronquíolos & 4 & $0,333-190$ & $3,33-1900$ \\
\hline Bronquíolos & 2,5 & $0,333-74$ & $3,33-740$ \\
\hline Bronquíolos & 1 & $0,333-35$ & $3,33-350$ \\
\hline Bronquíolos & 0,4 & $0,333-2$ & $3,33-20$ \\
\hline
\end{tabular}

Tabela B.1: Número de Reynolds em algumas vias aéreas humanas - Johnson [18] 


\section{Algoritmo de Thomas}

O método de Thomas é um método de eliminação desenvolvido para a solução de sistemas de equações algébricas lineares que apresentam matrizes de coeficientes tridiagonais. Este método simplifica significativamente os cálculos e não inclui o pivotamento, o qual destruiria a estrutura diagonal - Feyo [8].

Assim, para uma matriz tridiagonal de dimensão $n$

$$
A=\left[\begin{array}{llllllll}
b_{1} & c_{1} & 0 & 0 & \cdots & 0 & 0 & 0 \\
a_{2} & b_{2} & c_{2} & 0 & \cdots & 0 & 0 & 0 \\
0 & a_{3} & b_{3} & c_{3} & \cdots & 0 & 0 & 0 \\
\vdots & \vdots & \vdots & \vdots & \vdots & \vdots & \vdots & \vdots \\
0 & \cdots & \cdots & \cdots & \cdots & a_{n-1} & b_{n-1} & c_{n-1} \\
0 & \cdots & \cdots & \cdots & \cdots & 0 & a_{n} & b_{n}
\end{array}\right]
$$

em um sistema

$$
A x=d
$$

o algoritmo de Thomas se resume a:

1. Reduzir os termos da diagonal principal a um (1)

$$
1.1 \quad \begin{aligned}
w_{1} & =b_{1} \\
q_{1} & =\frac{c_{1}}{w_{1}} \\
g_{1} & =\frac{d_{1}}{w_{1}}
\end{aligned}
$$


1.2 Para $i=2,3, \ldots, n-1$ :

$$
\begin{aligned}
w_{i} & =b_{i}-a_{i} q_{i-1} \\
q_{i} & =\frac{c_{i}}{w_{i}} \\
g_{i} & =\frac{d_{i}-a_{i} g_{i-1}}{w_{i}}
\end{aligned}
$$

$1.3 w_{n}=b_{n}-a_{n} q_{n-1}$

$$
g_{n}=\frac{d_{n}-a_{n} g_{n-1}}{w_{n}}
$$

Desta forma, obtém-se o sistema linear

$$
\left[\begin{array}{llllll}
1 & q_{1} & 0 & \cdots & 0 & 0 \\
0 & 1 & q_{2} & \cdots & 0 & 0 \\
\vdots & \vdots & \vdots & \vdots & \vdots & \vdots \\
0 & 0 & \cdots & \cdots & 1 & q_{n-1} \\
0 & 0 & \cdots & \cdots & 0 & 1
\end{array}\right]\left[\begin{array}{l}
x_{1} \\
x_{2} \\
\vdots \\
x_{n-1} \\
x_{n}
\end{array}\right]=\left[\begin{array}{l}
g_{1} \\
g_{2} \\
\vdots \\
g_{n-1} \\
g_{n}
\end{array}\right]
$$

equivalente a $A x=d$.

2. Efetuar substituição reversa

$2.1 \quad x_{n}=g_{n}$

2.2 Para $i=n-1, n-2, \ldots, 1$ :

$$
x_{i}=g_{i}-q_{i} x_{i+1}
$$




\section{Avaliação da pressão $\bar{P}_{m}$, da}

\section{resistência $R_{m}$ e do suporte $h_{O}$ para}

$k=5$

\begin{tabular}{|l|l|l|l|l|l|}
\hline $\mathbf{k}_{\mathbf{m a x}}$ & $\mathbf{h}_{\mathbf{o}}$ & $\overline{\mathbf{P}}_{\mathbf{m}}$ & $\mathbf{R}_{\mathbf{m}}$ & $\mathbf{d e t} \mathbf{A}$ & Observações \\
\hline $10^{6}$ & $\frac{1}{16}$ & $10^{3}$ & $10^{7}$ & $\approx 1.00 \times 10^{35}$ & Fronteira praticamente imóvel \\
\hline $10^{6}$ & $\frac{1}{16}$ & $10^{3}$ & $10^{6}$ & $\approx 1.01 \times 10^{30}$ & Pequena expansão da fronteira \\
\hline $10^{6}$ & $\frac{1}{16}$ & $10^{3}$ & $10^{5}$ & $\approx 1.09 \times 10^{25}$ & Expansão uniforme e controlada da fronteira \\
\hline $10^{6}$ & $\frac{1}{16}$ & $10^{3}$ & $10^{4}$ & $\approx 2.09 \times 10^{20}$ & $\begin{array}{l}\text { Grande expansão no } 1^{o} \text { quadro - Expansão im- } \\
\text { previsível }\end{array}$ \\
\hline $10^{6}$ & $\frac{1}{16}$ & $10^{3}$ & $10^{3}$ & $\approx 5.27 \times 10^{16}$ & Fronteira ausente - Instabilidade \\
\hline $10^{6}$ & $\frac{1}{16}$ & $10^{3}$ & $10^{2}$ & $\approx 1.58 \times 10^{15}$ & Fronteira ausente - Instabilidade \\
\hline $10^{6}$ & $\frac{1}{16}$ & $10^{3}$ & 10 & $\approx 8.45 \times 10^{14}$ & Fronteira ausente - Instabilidade \\
\hline $10^{6}$ & $\frac{1}{16}$ & $10^{3}$ & 1 & $\approx 7.88 \times 10^{14}$ & Fronteira ausente - Instabilidade \\
\hline $10^{6}$ & $\frac{1}{16}$ & $10^{3}$ & 0 & $\approx 7.82 \times 10^{14}$ & Fronteira ausente - Instabilidade \\
\hline
\end{tabular}

Tabela D.1: Variação de $R_{m}$ para $\bar{P}_{m}=10^{3}, h_{o}=\frac{1}{16}$ e $\mathrm{k}_{m a x}=10^{6}$ 
114 Avaliação da pressão $\bar{P}_{m}$, da resistência $R_{m}$ e do suporte $h_{o}$ para $k=5$

\begin{tabular}{|l|l|l|l|l|l|}
\hline $\mathbf{k}_{\mathbf{m a x}}$ & $\mathbf{h}_{\mathbf{o}}$ & $\overline{\mathbf{P}}_{\mathbf{m}}$ & $\mathbf{R}_{\mathbf{m}}$ & detA & Observações \\
\hline $10^{6}$ & $\frac{1}{32}$ & $10^{3}$ & $10^{9}$ & overflow & Fronteira praticamente imóvel \\
\hline $10^{6}$ & $\frac{1}{32}$ & $10^{3}$ & $10^{8}$ & overflow & Fronteira praticamente imóvel \\
\hline $10^{6}$ & $\frac{1}{32}$ & $10^{3}$ & $10^{7}$ & $\approx 1.00 \times 10^{35}$ & Fronteira praticamente imóvel \\
\hline $10^{6}$ & $\frac{1}{32}$ & $10^{3}$ & $10^{6}$ & $\approx 1.01 \times 10^{30}$ & Pequena expansão da fronteira \\
\hline $10^{6}$ & $\frac{1}{32}$ & $10^{3}$ & $10^{5}$ & $\approx 1.15 \times 10^{25}$ & Expansão uniforme e controlada da fronteira \\
\hline $10^{6}$ & $\frac{1}{32}$ & $10^{3}$ & $10^{4}$ & $\approx 3.02 \times 10^{20}$ & $\begin{array}{l}\text { Grande expansão no } 1^{\circ} \text { quadro - Expansão im- } \\
\text { previsível }\end{array}$ \\
\hline $10^{6}$ & $\frac{1}{32}$ & $10^{3}$ & $10^{3}$ & $\approx 2.34 \times 10^{17}$ & Fronteira ausente - Instabilidade \\
\hline $10^{6}$ & $\frac{1}{32}$ & $10^{3}$ & $10^{2}$ & $\approx 2.42 \times 10^{16}$ & Fronteira ausente - Instabilidade \\
\hline $10^{6}$ & $\frac{1}{32}$ & $10^{3}$ & 10 & $\approx 1.77 \times 10^{16}$ & Fronteira ausente - Instabilidade \\
\hline $10^{6}$ & $\frac{1}{32}$ & $10^{3}$ & 1 & $\approx 1.71 \times 10^{16}$ & Fronteira ausente - Instabilidade \\
\hline $10^{6}$ & $\frac{1}{32}$ & $10^{3}$ & 0 & $\approx 1.70 \times 10^{16}$ & Fronteira ausente - Instabilidade \\
\hline
\end{tabular}

Tabela D.2: Variação de $R_{m}$ para $\bar{P}_{m}=10^{3}, h_{o}=\frac{1}{32}$ e $\mathrm{k}_{\max }=10^{6}$

\begin{tabular}{|l|l|l|l|l|l|}
\hline $\mathbf{k}_{\max }$ & $\mathbf{h}_{\mathbf{o}}$ & $\overline{\mathbf{P}}_{\mathbf{m}}$ & $\mathbf{R}_{\mathbf{m}}$ & $\operatorname{det} \mathbf{A}$ & Observações \\
\hline $10^{6}$ & $\frac{1}{64}$ & $10^{3}$ & $10^{8}$ & overflow & Fronteira praticamente imóvel \\
\hline $10^{6}$ & $\frac{1}{64}$ & $10^{3}$ & $10^{7}$ & $\approx 1.00 \times 10^{35}$ & Pequena expansão da fronteira \\
\hline $10^{6}$ & $\frac{1}{64}$ & $10^{3}$ & $10^{6}$ & $\approx 1.04 \times 10^{30}$ & Pequena expansão da fronteira \\
\hline $10^{6}$ & $\frac{1}{64}$ & $10^{3}$ & $10^{5}$ & $\approx 1.44 \times 10^{25}$ & Expansão uniforme e controlada da fronteira \\
\hline $10^{6}$ & $\frac{1}{64}$ & $10^{3}$ & $10^{4}$ & $\approx 1.22 \times 10^{21}$ & Fronteira ausente - Instabilidade \\
\hline $10^{6}$ & $\frac{1}{64}$ & $10^{3}$ & $10^{3}$ & $\approx 1.23 \times 10^{19}$ & Fronteira ausente - Instabilidade \\
\hline $10^{6}$ & $\frac{1}{64}$ & $10^{3}$ & $10^{2}$ & $\approx 4.98 \times 10^{18}$ & Fronteira ausente - Instabilidade \\
\hline $10^{6}$ & $\frac{1}{64}$ & $10^{3}$ & 10 & $\approx 4.5 \times 10^{18}$ & Fronteira ausente - Instabilidade \\
\hline $10^{6}$ & $\frac{1}{64}$ & $10^{3}$ & 1 & $\approx 4.45 \times 10^{18}$ & Fronteira ausente - Instabilidade \\
\hline $10^{6}$ & $\frac{1}{64}$ & $10^{3}$ & 0 & $\approx 4.45 \times 10^{18}$ & Fronteira ausente - Instabilidade \\
\hline
\end{tabular}

Tabela D.3: Variação de $R_{m}$ para $\bar{P}_{m}=10^{3}, h_{o}=\frac{1}{64}$ e $\mathrm{k}_{\max }=10^{6}$ 


\begin{tabular}{|l|l|l|l|l|l|}
\hline $\mathbf{k}_{\mathbf{m a x}}$ & $\mathbf{h}_{\mathbf{o}}$ & $\overline{\mathbf{P}}_{\mathbf{m}}$ & $\mathbf{R}_{\mathbf{m}}$ & $\mathbf{d e t} \mathbf{A}$ & Observações \\
\hline $10^{6}$ & $\frac{1}{16}$ & $10^{2}$ & $10^{6}$ & $\approx 1.01 \times 10^{30}$ & Fronteira praticamente imóvel \\
\hline $10^{6}$ & $\frac{1}{16}$ & $10^{2}$ & $10^{5}$ & $\approx 1.09 \times 10^{25}$ & Pequena expansão da fronteira \\
\hline $10^{6}$ & $\frac{1}{16}$ & $10^{2}$ & $10^{4}$ & $\approx 2.09 \times 10^{20}$ & Expansão uniforme e controlada da fronteira \\
\hline $10^{6}$ & $\frac{1}{16}$ & $10^{2}$ & $10^{3}$ & $\approx 5.27 \times 10^{16}$ & Fronteira ausente - Instabilidade \\
\hline $10^{6}$ & $\frac{1}{16}$ & $10^{2}$ & $10^{2}$ & $\approx 1.58 \times 10^{15}$ & Fronteira ausente - Instabilidade \\
\hline $10^{6}$ & $\frac{1}{16}$ & $10^{2}$ & 10 & $\approx 8.45 \times 10^{14}$ & Fronteira ausente - Instabilidade \\
\hline $10^{6}$ & $\frac{1}{16}$ & $10^{2}$ & 1 & $\approx 7.88 \times 10^{14}$ & Fronteira ausente - Instabilidade \\
\hline $10^{6}$ & $\frac{1}{16}$ & $10^{2}$ & 0 & $\approx 7.82 \times 10^{14}$ & Fronteira ausente - Instabilidade \\
\hline
\end{tabular}

Tabela D.4: Variação de $R_{m}$ para $\bar{P}_{m}=10^{2}, h_{o}=\frac{1}{16}$ e $\mathrm{k}_{\max }=10^{6}$

\begin{tabular}{|l|l|l|l|l|l|}
\hline $\mathbf{k}_{\max }$ & $\mathbf{h}_{\mathbf{o}}$ & $\overline{\mathbf{P}}_{\mathbf{m}}$ & $\mathbf{R}_{\mathbf{m}}$ & $\operatorname{det} \mathbf{A}$ & Observações \\
\hline $10^{6}$ & $\frac{1}{32}$ & $10^{2}$ & $10^{7}$ & $\approx 1.00 \times 10^{35}$ & Fronteira praticamente imóvel \\
\hline $10^{6}$ & $\frac{1}{32}$ & $10^{2}$ & $10^{6}$ & $\approx 1.01 \times 10^{30}$ & Fronteira praticamente imóvel \\
\hline $10^{6}$ & $\frac{1}{32}$ & $10^{2}$ & $10^{5}$ & $\approx 1.15 \times 10^{25}$ & Pequena expansão da fronteira \\
\hline $10^{6}$ & $\frac{1}{32}$ & $10^{2}$ & $10^{4}$ & $\approx 3.02 \times 10^{20}$ & Expansão uniforme e controlada da fronteira \\
\hline $10^{6}$ & $\frac{1}{32}$ & $10^{2}$ & $10^{3}$ & $\approx 2.34 \times 10^{17}$ & Fronteira ausente - Instabilidade \\
\hline $10^{6}$ & $\frac{1}{32}$ & $10^{2}$ & $10^{2}$ & $\approx 2.42 \times 10^{16}$ & Fronteira ausente - Instabilidade \\
\hline $10^{6}$ & $\frac{1}{32}$ & $10^{2}$ & 10 & $\approx 1.77 \times 10^{16}$ & Fronteira ausente - Instabilidade \\
\hline $10^{6}$ & $\frac{1}{32}$ & $10^{2}$ & 1 & $\approx 1.71 \times 10^{16}$ & Fronteira ausente - Instabilidade \\
\hline $10^{6}$ & $\frac{1}{32}$ & $10^{2}$ & 0 & $\approx 1.70 \times 10^{16}$ & Fronteira ausente - Instabilidade \\
\hline
\end{tabular}

Tabela D.5: Variação de $R_{m}$ para $\bar{P}_{m}=10^{2}, h_{o}=\frac{1}{32}$ e $\mathrm{k}_{\max }=10^{6}$ 
116 Avaliação da pressão $\bar{P}_{m}$, da resistência $R_{m}$ e do suporte $h_{o}$ para $k=5$

\begin{tabular}{|l|l|l|l|l|l|}
\hline $\mathbf{k}_{\max }$ & $\mathbf{h}_{\mathbf{o}}$ & $\overline{\mathbf{P}}_{\mathbf{m}}$ & $\mathbf{R}_{\mathbf{m}}$ & $\mathbf{d e t} \mathbf{A}$ & Observaçoes \\
\hline $10^{6}$ & $\frac{1}{64}$ & $10^{2}$ & $10^{6}$ & $\approx 1.04 \times 10^{30}$ & Fronteira praticamente imóvel \\
\hline $10^{6}$ & $\frac{1}{64}$ & $10^{2}$ & $10^{5}$ & $\approx 1.44 \times 10^{25}$ & Pequena expansão da fronteira \\
\hline $10^{6}$ & $\frac{1}{64}$ & $10^{2}$ & $10^{4}$ & $\approx 1.22 \times 10^{21}$ & $\begin{array}{l}\text { Grande expansão no } 1^{\circ} \text { quadro - Expansão uni- } \\
\text { forme e controlada da fronteira }\end{array}$ \\
\hline $10^{6}$ & $\frac{1}{64}$ & $10^{2}$ & $10^{3}$ & $\approx 1.23 \times 10^{19}$ & Fronteira ausente - Instabilidade \\
\hline $10^{6}$ & $\frac{1}{64}$ & $10^{2}$ & $10^{2}$ & $\approx 4.98 \times 10^{18}$ & Fronteira ausente - Instabilidade \\
\hline $10^{6}$ & $\frac{1}{64}$ & $10^{2}$ & 10 & $\approx 4.5 \times 10^{18}$ & Fronteira ausente - Instabilidade \\
\hline $10^{6}$ & $\frac{1}{64}$ & $10^{2}$ & 1 & $\approx 4.45 \times 10^{18}$ & Fronteira ausente - Instabilidade \\
\hline $10^{6}$ & $\frac{1}{64}$ & $10^{2}$ & 0 & $\approx 4.45 \times 10^{18}$ & Fronteira ausente - Instabilidade \\
\hline
\end{tabular}

Tabela D.6: Variação de $R_{m}$ para $\bar{P}_{m}=10^{2}, h_{o}=\frac{1}{64}$ e $\mathrm{k}_{\max }=10^{6}$

\begin{tabular}{|l|l|l|l|l|l|}
\hline $\mathbf{k}_{\max }$ & $\mathbf{h}_{\mathbf{o}}$ & $\overline{\mathbf{P}}_{\mathbf{m}}$ & $\mathbf{R}_{\mathbf{m}}$ & $\operatorname{det} \mathbf{A}$ & Observações \\
\hline $10^{6}$ & $\frac{1}{16}$ & 10 & $10^{5}$ & $\approx 1.09 \times 10^{25}$ & Fronteira praticamente imóvel \\
\hline $10^{6}$ & $\frac{1}{16}$ & 10 & $10^{4}$ & $\approx 2.09 \times 10^{20}$ & Pequena expansão da fronteira \\
\hline $10^{6}$ & $\frac{1}{16}$ & 10 & $10^{3}$ & $\approx 5.27 \times 10^{16}$ & Fronteira ausente - Instabilidade \\
\hline $10^{6}$ & $\frac{1}{16}$ & 10 & $10^{2}$ & $\approx 1.58 \times 10^{15}$ & Fronteira ausente - Instabilidade \\
\hline $10^{6}$ & $\frac{1}{16}$ & 10 & 10 & $\approx 8.45 \times 10^{14}$ & Fronteira ausente - Instabilidade \\
\hline $10^{6}$ & $\frac{1}{16}$ & 10 & 1 & $\approx 7.88 \times 10^{14}$ & Fronteira ausente - Instabilidade \\
\hline $10^{6}$ & $\frac{1}{16}$ & 10 & 0 & $\approx 7.82 \times 10^{14}$ & Fronteira ausente - Instabilidade \\
\hline
\end{tabular}

Tabela D.7: Variação de $R_{m}$ para $\bar{P}_{m}=10, h_{o}=\frac{1}{16}$ e $\mathrm{k}_{\max }=10^{6}$ 


\begin{tabular}{|l|l|l|l|l|l|}
\hline $\mathbf{k}_{\max }$ & $\mathbf{h}_{\mathbf{o}}$ & $\overline{\mathbf{P}}_{\mathbf{m}}$ & $\mathbf{R}_{\mathbf{m}}$ & $\operatorname{det} \mathbf{A}$ & Observações \\
\hline $10^{6}$ & $\frac{1}{32}$ & 10 & $10^{6}$ & $\approx 1.01 \times 10^{30}$ & Fronteira praticamente imóvel \\
\hline $10^{6}$ & $\frac{1}{32}$ & 10 & $10^{5}$ & $\approx 1.15 \times 10^{25}$ & Fronteira praticamente imóvel \\
\hline $10^{6}$ & $\frac{1}{32}$ & 10 & $10^{4}$ & $\approx 3.02 \times 10^{20}$ & Pequena expansão da fronteira \\
\hline $10^{6}$ & $\frac{1}{32}$ & 10 & $10^{3}$ & $\approx 2.34 \times 10^{17}$ & Fronteira ausente - Instabilidade \\
\hline $10^{6}$ & $\frac{1}{32}$ & 10 & $10^{2}$ & $\approx 2.42 \times 10^{16}$ & Fronteira ausente - Instabilidade \\
\hline $10^{6}$ & $\frac{1}{32}$ & 10 & 10 & $\approx 1.77 \times 10^{16}$ & Fronteira ausente - Instabilidade \\
\hline $10^{6}$ & $\frac{1}{32}$ & 10 & 1 & $\approx 1.71 \times 10^{16}$ & Fronteira ausente - Instabilidade \\
\hline $10^{6}$ & $\frac{1}{32}$ & 10 & 0 & $\approx 1.70 \times 10^{16}$ & Fronteira ausente - Instabilidade \\
\hline
\end{tabular}

Tabela D.8: Variação de $R_{m}$ para $\bar{P}_{m}=10, h_{o}=\frac{1}{32}$ e $\mathrm{k}_{\max }=10^{6}$

\begin{tabular}{|l|l|l|l|l|l|}
\hline $\mathbf{k}_{\max }$ & $\mathbf{h}_{\mathbf{o}}$ & $\overline{\mathbf{P}}_{\mathbf{m}}$ & $\mathbf{R}_{\mathbf{m}}$ & $\operatorname{det} \mathbf{A}$ & Observações \\
\hline $10^{6}$ & $\frac{1}{64}$ & 10 & $10^{5}$ & $\approx 1.44 \times 10^{25}$ & Fronteira praticamente imóvel \\
\hline $10^{6}$ & $\frac{1}{64}$ & 10 & $10^{4}$ & $\approx 1.22 \times 10^{21}$ & Pequena expansão da fronteira \\
\hline $10^{6}$ & $\frac{1}{64}$ & 10 & $10^{3}$ & $\approx 1.23 \times 10^{19}$ & Fronteira ausente - Instabilidade \\
\hline $10^{6}$ & $\frac{1}{64}$ & 10 & $10^{2}$ & $\approx 4.98 \times 10^{18}$ & Fronteira ausente - Instabilidade \\
\hline $10^{6}$ & $\frac{1}{64}$ & 10 & 10 & $\approx 4.5 \times 10^{18}$ & Fronteira ausente - Instabilidade \\
\hline $10^{6}$ & $\frac{1}{64}$ & 10 & 1 & $\approx 4.45 \times 10^{18}$ & Fronteira ausente - Instabilidade \\
\hline $10^{6}$ & $\frac{1}{64}$ & 10 & 0 & $\approx 4.45 \times 10^{18}$ & Fronteira ausente - Instabilidade \\
\hline
\end{tabular}

Tabela D.9: Variação de $R_{m}$ para $\bar{P}_{m}=10, h_{o}=\frac{1}{64}$ e $\mathrm{k}_{\max }=10^{6}$ 
118 Avaliação da pressão $\bar{P}_{m}$, da resistência $R_{m}$ e do suporte $h_{\circ}$ para $k=5$

\begin{tabular}{|l|l|l|l|l|l|}
\hline $\mathbf{k}_{\max }$ & $\mathbf{h}_{\mathbf{o}}$ & $\overline{\mathbf{P}}_{\mathbf{m}}$ & $\mathbf{R}_{\mathbf{m}}$ & $\mathbf{d e t} \mathbf{A}$ & Observações \\
\hline $10^{6}$ & $\frac{1}{16}$ & 7 & $7 \times 10^{2}$ & $\approx 2.22 \times 10^{16}$ & Fronteira ausente - Instabilidade \\
\hline $10^{6}$ & $\frac{1}{16}$ & 5 & $5 \times 10^{2}$ & $\approx 1.10 \times 10^{16}$ & Fronteira ausente - Instabilidade \\
\hline $10^{6}$ & $\frac{1}{16}$ & 2 & 5 & $\approx 8.13 \times 10^{14}$ & Fronteira ausente - Instabilidade \\
\hline $10^{6}$ & $\frac{1}{16}$ & 1 & $10^{3}$ & $\approx 5.27 \times 10^{16}$ & Fronteira ausente - Instabilidade \\
\hline $10^{6}$ & $\frac{1}{16}$ & 1 & $10^{2}$ & $\approx 1.58 \times 10^{15}$ & Fronteira ausente - Instabilidade \\
\hline $10^{6}$ & $\frac{1}{16}$ & 1 & 10 & $\approx 8.45 \times 10^{14}$ & Fronteira ausente - Instabilidade \\
\hline $10^{6}$ & $\frac{1}{16}$ & 1 & 1 & $\approx 7.88 \times 10^{14}$ & Fronteira ausente - Instabilidade \\
\hline $10^{6}$ & $\frac{1}{16}$ & 1 & 0 & $\approx 7.82 \times 10^{14}$ & Fronteira ausente - Instabilidade \\
\hline $10^{6}$ & $\frac{1}{16}$ & 0 & $10^{2}$ & $\approx 1.58 \times 10^{15}$ & Fronteira ausente - Instabilidade \\
\hline $10^{6}$ & $\frac{1}{16}$ & 0 & 10 & $\approx 8.45 \times 10^{14}$ & Fronteira ausente - Instabilidade \\
\hline $10^{6}$ & $\frac{1}{16}$ & 0 & 0 & $\approx 7.82 \times 10^{14}$ & Fronteira ausente - Instabilidade \\
\hline
\end{tabular}

Tabela D.10: Variação de $R_{m}$ para $\bar{P}_{m}$ variando de 10 a $0, h_{o}=\frac{1}{16}$ e $\mathrm{k}_{m a x}=10^{6}$

\begin{tabular}{|l|l|l|l|l|l|}
\hline $\mathbf{k}_{\mathbf{m a x}}$ & $\mathbf{h}_{\mathbf{o}}$ & $\overline{\mathbf{P}}_{\mathbf{m}}$ & $\mathbf{R}_{\mathbf{m}}$ & $\operatorname{det} \mathbf{A}$ & Observações \\
\hline $10^{6}$ & $\frac{1}{32}$ & 7 & $7 \times 10^{2}$ & $\approx 1.25 \times 10^{17}$ & Fronteira ausente - Instabilidade \\
\hline $10^{6}$ & $\frac{1}{32}$ & 5 & $5 \times 10^{2}$ & $\approx 7.75 \times 10^{16}$ & Fronteira ausente - Instabilidade \\
\hline $10^{6}$ & $\frac{1}{32}$ & 2 & 5 & $\approx 1.74 \times 10^{16}$ & Fronteira ausente - Instabilidade \\
\hline $10^{6}$ & $\frac{1}{32}$ & 1 & $10^{3}$ & $\approx 2.34 \times 10^{17}$ & Fronteira ausente - Instabilidade \\
\hline $10^{6}$ & $\frac{1}{32}$ & 1 & $10^{2}$ & $\approx 2.42 \times 10^{16}$ & Fronteira ausente - Instabilidade \\
\hline $10^{6}$ & $\frac{1}{32}$ & 1 & 10 & $\approx 1.77 \times 10^{16}$ & Fronteira ausente - Instabilidade \\
\hline $10^{6}$ & $\frac{1}{32}$ & 1 & 1 & $\approx 1.71 \times 10^{16}$ & Fronteira ausente - Instabilidade \\
\hline $10^{6}$ & $\frac{1}{32}$ & 1 & 0 & $\approx 1.70 \times 10^{16}$ & Fronteira ausente - Instabilidade \\
\hline $10^{6}$ & $\frac{1}{32}$ & 0 & $10^{2}$ & $\approx 2.42 \times 10^{16}$ & Fronteira ausente - Instabilidade \\
\hline $10^{6}$ & $\frac{1}{32}$ & 0 & 10 & $\approx 1.77 \times 10^{16}$ & Fronteira ausente - Instabilidade \\
\hline $10^{6}$ & $\frac{1}{32}$ & 0 & 0 & $\approx 1.70 \times 10^{16}$ & Fronteira ausente - Instabilidade \\
\hline
\end{tabular}

Tabela D.11: Variação de $R_{m}$ para $\bar{P}_{m}$ variando de 10 a $0, h_{o}=\frac{1}{32}$ e $\mathrm{k}_{\max }=10^{6}$ 


\begin{tabular}{|l|l|l|l|l|l|}
\hline $\mathbf{k}_{\mathbf{m a x}}$ & $\mathbf{h}_{\mathbf{o}}$ & $\overline{\mathbf{P}}_{\mathbf{m}}$ & $\mathbf{R}_{\mathbf{m}}$ & $\operatorname{det} \mathbf{A}$ & Observações \\
\hline $10^{6}$ & $\frac{1}{64}$ & 7 & $7 \times 10^{2}$ & $\approx 9.27 \times 10^{18}$ & Fronteira ausente - Instabilidade \\
\hline $10^{6}$ & $\frac{1}{64}$ & 5 & $5 \times 10^{2}$ & $\approx 7.61 \times 10^{18}$ & Fronteira ausente - Instabilidade \\
\hline $10^{6}$ & $\frac{1}{64}$ & 2 & 5 & $\approx 4.47 \times 10^{18}$ & Fronteira ausente - Instabilidade \\
\hline $10^{6}$ & $\frac{1}{64}$ & 1 & $10^{3}$ & $\approx 4.98 \times 10^{18}$ & Fronteira ausente - Instabilidade \\
\hline $10^{6}$ & $\frac{1}{64}$ & 1 & $10^{2}$ & $\approx 4.5 \times 10^{18}$ & Fronteira ausente - Instabilidade \\
\hline $10^{6}$ & $\frac{1}{64}$ & 1 & 10 & $\approx 4.45 \times 10^{18}$ & Fronteira ausente - Instabilidade \\
\hline $10^{6}$ & $\frac{1}{64}$ & 1 & 1 & $\approx 4.45 \times 10^{18}$ & Fronteira ausente - Instabilidade \\
\hline $10^{6}$ & $\frac{1}{64}$ & 1 & 0 & $\approx 4.98 \times 10^{18}$ & Fronteira ausente - Instabilidade \\
\hline $10^{6}$ & $\frac{1}{64}$ & 0 & $10^{2}$ & $\approx 4.5 \times 10^{18}$ & Fronteira ausente - Instabilidade \\
\hline $10^{6}$ & $\frac{1}{64}$ & 0 & 80 & $\approx 4.45 \times 10^{18}$ & Fronteira ausente - Instabilidade \\
\hline
\end{tabular}

Tabela D.12: Variação de $R_{m}$ para $\bar{P}_{m}$ variando de 10 a $0, h_{o}=\frac{1}{64}$ e $\mathrm{k}_{\max }=10^{6}$ 
120 Avaliação da pressão $\bar{P}_{m}$, da resistência $R_{m}$ e do suporte $h_{o}$ para $k=5$ 


\section{Referências Bibliográficas}

[1] ARTHURS, Kayne M.; MOORE, Leon C.; PESKIN, Charles S.; PITMAN, E. Bruce; LAYTON, H. E. Modeling arteriolar flow and mass transport using the immersed boundary method. Journal of Computacional Physics, v. 147, p. 402-440, 1998.

[2] BEVILACQUA, Joyce S. Análise numérica II - Notas de aula. Instituto de Matemática e Estatística da Universidade de São Paulo, 1999.

[3] BURDEN, Richard L.; FAIRES, J. Douglas. Numerical analysis. 6th ed. Pacific Grove : Brooks/Cole, 1997.

[4] CHORIN, Alexandre J. Numerical solution of the Navier-Stokes equations. Mathematics of Computation, v. 22, n. 104, p. 745-762, 1968.

[5] CORPO HUMANO.http://br.geocities.com/corpo_humano/sistema_respiratório.htm

[6] DUPUIS, Yvon G. Basic concepts. In:- Ventilators: theory and clinical application. St. Louis : Mosby-Year Book, 1992. p. 3-29.

[7] ENCICLOPÉDIA ENCARTA. http://encarta.msn.com/index/conciseindex/64/06485000.htm? $\mathrm{z}=1 \& \mathrm{pg}=2 \& \mathrm{br}=1$

[8] FACULDADE DE ENGENHARIA DA UNIVERSIDADE DO PORTO - PORTUGAL. http://www.fe.up.pt/ sfeyo/mn/Thomas.pdf

[9] FAUCI, Lisa J. Computacional modeling of the swimming of biflagellated algal cells. Contemporary Mathematics, v. 141, p. 91-102, 1993. 
[10] FAUCI, Lisa J.; FOGELSON, Aaron L. Truncated Newton methods and the modeling of complex immersed elastic structures. Comunications on Pure and Applied Mathematics, v. XLVI, p. 787-818, 1993.

[11] FAUCI, Lisa J.; PESKIN, Charles S. A Computacional model of aquatic animal locomotion. Journal of Computacional Physics, v. 77, p. 85-108, 1988.

[12] FOGELSON, A. L. A mathematical model and numerical method for studying platelet adhesion and aggregation during blood clotting. Journal of Computacional Physics, v. 56, p. 111-134, 1984.

[13] FORTUNA, Armando de Oliveira. Técnicas computacionais para dinâmica dos fluidos: conceitos básicos e aplicações. São Paulo : Editora da Universidade de São Paulo, 2000.

[14] GERALD, Curtis F.; WHEATLEY, Patrick O. Applied numerical analysis. 5th ed. [S.1.] : Addison-Wesley, 1994.

[15] HALLIDAY, David; RESNICK, Robert. Physics for students of science and engineering. New York : John Wiley \& Sons, 1964.

[16] JACKSON, Andrew C; MILHORN Jr., Howard T. Digital computer simulation of respiratory mechanics. Computers and Biomedical Research, v. 6, p. 27-56, 1973.

[17] JOHNS HOPKINS SCHOOL OF MEDICINE'S. Interactive Respiratory Physiology. http://omie.med.jhmi.edu/res_phys/Encyclopedia/

[18] JOHNSON, Arthur T. Respiratory responses. In: - - Biomechanics and exercise physiology. New York: John Wiley \& Sons, 1991. p. 166-360.

[19] KERNiGHAN, Brian W.; RITCHIE, Dennis M. C - A linguagem de programação. Rio de Janeiro : Campus, 1986.

[20] MEAD, Jere. Mechanical properties of lungs. Physiological Reviews, Boston, v. 41, n. 2, p. 281-330, Apr. 1961.

[21] MING-CHIH, Lai. Simulation of the flow past an array of circular cylinders as a test of the immersed boundary method. New York, 1998. Ph.D. thesis - Department of Mathematics, New York University. 
[22] OTIS, A. B.; PROCTOR, D. F. Measurement of alveolar pressure in human subjects. American Journal of Physiology, v. 152, p. 106-112, 1948.

[23] PESKIN, Charles S. Flow patterns around heart valves: a digital computer method for solving the equations of motion. New York, 1972. Ph.D. thesis - Einstein College of Medicine, Yeshiva University.

[24] —. Flow patterns around heart valves: a numerical method. Journal of Computacional Physics, v. 10, p. 252-271, 1972.

[25] - . Numerical analysis of blood flow in the heart. Journal of Computacional Physics, v. 25, n. 3 , p. $220-252,1977$.

[26] PESKIN, Charles S.; PRINTZ, Beth F. Improved volume conservation in the computation of flows with immersed elastic boundaries. Journal of Computacional Physics, v. 105, n. 1, p. 33-46, Mar. 1993.

[27] PEYRET, Roger; TAYLOR, Thomas D. Computacional methods for fluid flow. New York : Springer-Verlag, 1983.

[28] PRESS, William H. et al. Numerical recipes in $C$ : the art of scientific computing. 2nd ed. New York : Cambridge University Press, 1992.

[29] REMIGIO, Santos A. E. Introdução de fontes e sumidouros em escoamentos incompressíveis bidimensionais por intermédio do método da fronteira imersa. São Paulo, 2000. Dissertação de Mestrado - Instituto de Matemática e Estatística, Universidade de São Paulo.

[30] RIBAS, Aymara F. et al. Normas para apresentação de trabalhos - Referências bibliográficas. v. 6. 6. ed. Curitiba : Universidade Ferderal do Paraná, 1996.

[31] ROMA, Alexandre M. A multilevel self adaptive version of the immersed boundary method. New York, 1994. Ph.D. thesis - Department of Mathematics, New York University.

[32] —. An adaptive immersed boundary method: implementation issues, applications and work in progress. Symposium on adaptive methods for partial differential equations, University of Utah, June 22-24, 1998. 
[33] ROSAR, Madeleine Elisabeth. A three-dimensional computer model for fluid flow through a collapsible tube. New York, 1994. Ph.D. thesis - Department of Mathematics, New York University.

[34] SCHILDT, Herbert. C completo e total. 3. ed. São Paulo : Makron Books, 1996.

[35] SEARS, Francis W.; ZEMANSKY, Mark W. Física. Rio de Janeiro : Livros Técnicos e Científicos, 1976. v. 1: Mecânica/Hidrodinâmica.

[36] SMITH, Jeffrey C.; STAMENOVIC, Dimitrije. Surface forces in lungs.I.Alveolar surface tension - lung volume relationships. Journal of Apllied Physiology, Boston, v. 60(4), p. 1341-1350, 1986.

[37] STAMENOVIC, Dimitrije; SMITH, Jeffrey C. Surface forces in lungs.II.Microestructural mechanics and lung stability. Journal of Applied Physiology, Boston, v. 60(4), p. 1351-1357, 1986.

[38] STOER, Josef; BULIRSCH, Roland. Introduction to numerical analysis. New York : Springer-Verlag, 1993.

[39] TU, C.; PESKIN, C. S. Stability and instability in the computation of flows with moving immersed boundaries: a comparison of three methods. SIAM Journal on Scientific and Statistical Computation, v. 13, p. 1361-1376, 1992.

[40] WEBANATOMY - UNIVERSITY OF MINNESOTA. http://www.gen.umn.edu/faculty_staff/jensen/1135/webanatomy/wa_respiratory/

[41] WEST, John B. Fisiologia respiratória moderna. 3. ed. São Paulo : Manole, 1986.

[42] YOSHIKAWA, Roberto M. S.; ROMA, Alexandre M. Estudo da interação fluidofronteira através do método da fronteira imersa. Simpósio de Iniciação Científica da Universidade de São Paulo, 6, v. 2, p. 235, 1998. 